SCIENTIFIC REPORT

\title{
Prevalence and causes of blindness and low vision in leprosy villages of north eastern Nigeria
}

\section{Mpyet, A W Solomon}

Br J Ophthalmol 2005;89:417-419. doi: 10.1136/bjo.2004.048777

\begin{abstract}
Aims: To determine the prevalence and spectrum of ocular pathology, and the prevalence and causes of blindness and low vision in leprosy villages of north eastern Nigeria.

Methods: People affected by leprosy, aged 30 years and above, resident in eight leprosy villages were invited to participate. Ocular examination was undertaken of each consenting individual.

Results: 480 people were examined. $456(48 \%)$ of 960 eyes had at least one ocular lesion, but only $37 \%$ of all lesions were leprosy related and potentially sight threatening. The prevalence of blindness (VA $<3 / 60$ with available correction) was $10.4 \%$. An additional $7.5 \%$ of subjects were severely visually impaired $(3 / 60 \leqslant V A<6 / 60)$. Cataract was the commonest cause of blindness. Other major causes were non-trachomatous corneal opacity and trachoma.

Conclusions: Blindness and low vision are highly prevalent among leprosy patients in this setting. Only a third of the burden of ocular pathology is related to the direct effects of leprosy. Efforts to reduce the backlog of cataract and trichiasis, to improve early detection and management of lagophthalmos, and to provide refractive services are urgently required.
\end{abstract}

n 2000, the most recent year for which global data are available, 719330 new cases of leprosy were registered, and leprosy was still considered a public health problem in 15 countries. ${ }^{1}$ At least 10 million people have been treated for leprosy with multidrug therapy (MDT: dapsone, rifampicin, clofazimine) worldwide. ${ }^{2}$ Before the introduction of MDT in the early 1980s, millions more received dapsone monotherapy. Successful completion of treatment, however, does not necessarily prevent development of long term sequelae. ${ }^{34}$ Disabilities in leprosy predominantly affect limbs and eyes, and result in social discrimination and rejection in many societies. Best current estimates suggest that there are about 200 000-300 000 blind people affected by leprosy (PAL). ${ }^{5}$

In order for leprosy control and blindness prevention programmes to be able to plan eye care services, data on the prevalence and causes of blindness and low vision in PALs are required. This study aims to determine these parameters for residents of leprosy villages in north eastern Nigeria.

\section{PATIENTS AND METHODS}

There are 13 leprosy villages in the 13 states of north eastern Nigeria. Eight (selected on the basis of accessibility) were included in this study. All residents of these villages aged 30 years or above were invited to participate if they: (i) were currently on MDT; (ii) had completed MDT, or (iii) had completed dapsone monotherapy. After explaining the purpose and conduct of the study, verbal consent was obtained. For each consenting individual, data on age, sex and duration since diagnosis of leprosy were recorded. The type of leprosy (multibacillary or paucibacillary) was determined from the subject's medical notes (where sufficient information had been recorded) or by inference from the subject's description of their treatment regimen. Visual acuity (VA) was assessed using the Snellen or illiterate E chart at 6 metres, using available correction for those with spectacles. Aphakic subjects had their VA assessed with a +10 (aphakic) lens if no spectacles were available. Definitions of blindness and visual impairment were: $\mathrm{VA}=6 / 18$, normal; $6 / 60=$ $\mathrm{VA}<6 / 18$, visual impairment; $3 / 60=\mathrm{VA}<6 / 60$, severe visual impairment; VA $<3 / 60$, blind. Subjects unable to see $6 / 18$ had their VA re-assessed with pinhole.

All subjects were then examined by an ophthalmologist (CM) using a pen torch and direct ophthalmoscope. Cataract was defined as the presence of lens opacity. Trachomatous corneal opacity was defined as the presence of corneal opacification in an eye with corrected or uncorrected entropion or trichiasis (including evidence of recent epilation). Non-trachomatous corneal opacity was defined as the presence of corneal opacity without corrected or uncorrected entropion or trichiasis. Glaucoma was defined as the presence of a pale, cupped disc with a cup to disc ratio of 0.8 or more. Aphakia was defined as absence of the lens. Uveitis was defined as the presence of active uveal inflammation or evidence of previous uveal inflammation, such as miosed pupil, posterior synechiae, or iris atrophy. Refractive error was defined as visual acuity $<6 / 18$ with available correction that improved to $6 / 18$ or better with pinhole. For those unable to see $6 / 18$ with pinhole, clinical judgment was used to determine the main cause of visual impairment or blindness. If two or more pathologies were adjudged to contribute equally, visual impairment or blindness was attributed to the most treatable cause.

Data were entered in Epi-Info 6.04d (World Health Organization, Geneva, Switzerland), and analysed in EpiInfo and Stata 7 (Stata Corp, College Station, TX, USA). Using multiple logistic regression, age, sex, time since diagnosis, and type of leprosy were modelled as potential risk factors for being functionally blind.

Individuals identified as having treatable ophthalmic conditions were offered free surgery and/or medical care, as appropriate. The study was approved by ethics committees of the Jos University Teaching Hospital and the London School of Hygiene and Tropical Medicine.

\section{RESULTS}

A total of 480 people met the inclusion criteria and were examined. No eligible individual present in the villages during the study refused participation. Some residents may have declined to participate by being absent. A total of 211 $(44.0 \%)$ females and $269(56.0 \%)$ males were seen; ages

Abbreviations: MDT, multidrug therapy; PAL, people affected by leprosy; VA, visual acuity 
Table 1 Ocular lesions in the study population (480 subjects, 960 eyes)

\begin{tabular}{lcc}
\hline Lesion & No of eyes with lesion & $\begin{array}{l}\text { Prevalence of lesion among all } \\
\text { eyes (\%) }\end{array}$ \\
\hline Lens opacity & 321 & 33.4 \\
Corneal opacity & 149 & 15.5 \\
Lagophthalmos $<6 \mathrm{~mm}$ & 94 & 9.8 \\
Lagophthalmos $>6 \mathrm{~mm}$ & 27 & 2.8 \\
Entropion/trichiasis & 89 & 9.3 \\
Refractive error & 50 & 5.2 \\
Retinal pathology & 34 & 3.5 \\
Uveitis & 21 & 2.2 \\
Total number of lesions & 785 (in 456 eyes) \\
\hline
\end{tabular}

Table 2 Visual status of study subjects

\begin{tabular}{lcc}
\hline Vision & Number of subjects & $\%(95 \% \mathrm{Cl})$ \\
\hline Normal & 325 & $67.7(63.3$ to 71.8$)$ \\
Visual impairment & 69 & $14.4(11.4$ to 17.9$)$ \\
Severe visual impairment & 36 & $7.5(5.4$ to 10.3$)$ \\
Blind & 50 & $10.4(7.9$ to 13.6$)$ \\
Total & 480 & 100 \\
\hline
\end{tabular}

ranged from 30 to 96 years. Of 480 subjects, 90 (18.8\%; 37 females, 53 males) were classified as having paucibacillary leprosy; 230 (47.9\%; 95 females, 135 males) were classified as having multibacillary leprosy. The type of leprosy could not be determined in 160 (33.3\%) individuals.

\section{Ophthalmic pathology}

Of 960 eyes, $456(47.5 \%)$ eyes had at least one pathology; many eyes had more than one lesion. The commonest problem was lens opacification, which was seen in one in three eyes (table 1). Nearly one eye in 10 had entropion or trichiasis. More than one eye in 20 had a refractive error that reduced VA to $<6 / 18$.

\section{Visual status}

The prevalence of functional blindness $\left(\mathrm{VA}<3 / 60^{6}\right)$ was $10.4 \%$ (95\% CI 7.9 to 13.6 ) (table 2). If blindness had been defined as $\mathrm{VA}<6 / 60$ (corresponding to grade 2 disability on the WHO scale ${ }^{7}$ ), the prevalence of blindness would have been $17.9 \%$ (95\% CI 14.6 to 21.7 ).

The WHO disability scale ${ }^{7}$ considers eyes rather than people. Of 960 eyes examined, 504 had grade 0 disability (no evidence of visual loss), 150 had grade 1 disability (evidence of visual loss, but VA 6/60 or better), and 306 had grade 2 disability ( severe visual impairment; VA<6/60).

\section{Causes of visual impairment and blindness}

Cataract was the commonest cause of both visual impairment and blindness, being responsible for $46 \%$ of blindness (table 3$)$. Nearly one third of the blind $(2.9 \%$ of all subjects examined) had bilateral non-trachomatous corneal opacity, and can therefore be considered blind due to direct complications of leprosy.

\section{Risk factors for blindness}

In logistic regression analyses, increasing age was the only identified risk factor for being functionally blind (table 4).

\section{DISCUSSION}

Most of the world's leprosy sufferers live in developing countries, where they are triply disadvantaged: the prevalence of many other diseases is high; medical care is very limited; and stigmatisation-an experience common to leprosy patients in all societies-limits use of the scarce medical services that are available. Resulting delays in treatment worsen long term outcomes.

Table 3 Main cause of any visual impairment (including visual impairment, severe visual impairment, and blindness) and main causes of blindness

\begin{tabular}{|c|c|c|c|c|}
\hline \multirow[b]{2}{*}{ Cause } & \multicolumn{2}{|c|}{ Any visual impairment (VI) } & \multicolumn{2}{|l|}{ Blindness } \\
\hline & $\begin{array}{l}\text { Number } \\
\text { (prevalence, \%) }\end{array}$ & $\begin{array}{l}\text { Proportion of } \\
\text { all cases of VI (\%) }\end{array}$ & $\begin{array}{l}\text { Number } \\
\text { (prevalence, \%) }\end{array}$ & $\begin{array}{l}\text { Proportion of all } \\
\text { cases of blindness } \\
\text { (\%) }\end{array}$ \\
\hline Cataract & 80 (17) & 52 & $23(5)$ & 46 \\
\hline $\begin{array}{l}\text { Non-trachomatous corneal } \\
\text { opacity }\end{array}$ & $26(5)$ & 17 & $14(3)$ & 28 \\
\hline Aphakia & $20(4)$ & 13 & $3(0.6)$ & 6 \\
\hline $\begin{array}{l}\text { Trachomatous corneal } \\
\text { opacity }\end{array}$ & $13(3)$ & 8 & $6(1)$ & 12 \\
\hline Glaucoma & $9(2)$ & 6 & $3(0.6)$ & 6 \\
\hline Others & $7(2)$ & 5 & $1(0.2)$ & 2 \\
\hline Total (all causes) & $155(32)$ & 100 & $50(10)$ & 100 \\
\hline
\end{tabular}




\begin{tabular}{|c|c|c|}
\hline "Exposure" & Exposed/seen (\%) & Adjusted odds ratio $(95 \% \mathrm{Cl})$ \\
\hline $\begin{array}{l}\text { Female } \\
\text { Age (for each year) } \\
>10 \text { years since diagnosis } \\
\text { Paucibacillary leprosy }\end{array}$ & $\begin{array}{l}211 / 480(44.0) \\
\text { Not applicable } \\
328 / 480(68.3) \\
90 / 320^{*}(28.1)\end{array}$ & $\begin{array}{l}0.86(0.36 \text { to } 2.07) \\
1.04(1.01 \text { to } 1.07) \\
1.56(0.46 \text { to } 5.28) \\
1.06(0.42 \text { to } 2.67)\end{array}$ \\
\hline
\end{tabular}

Nearly half of all eyes of leprosy village residents examined in this study had one or more identifiable ocular lesions. Using WHO's definition of functional blindness, more than one in $10(10.4 \%)$ were blind, and more than one in six $(17.9 \%)$ had VA $<6 / 60$. These very high prevalences of ocular pathology and blindness may be due to the relatively long history of leprosy in and advanced age of the subjects in this study, and the fact that five of eight study villages were in a trachoma endemic area. ${ }^{8}$ The prevalence of blindness among PALs elsewhere in Nigeria has been estimated at $2.9 \%$ in the north west, where blinding trachoma is found, ${ }^{9}$ and $9.7 \%$ in the south east, where trachoma does not constitute a public health problem. ${ }^{10}$

As is generally true in PALs, ${ }^{11}$ cataract was the commonest cause of blindness. Provision of an adequate cataract surgical service could nearly halve the burden of blindness in this population. Corneal opacity was the second most common blinding condition: $28 \%$ of the blind had corneal opacity without associated trichiasis or entropion, while $12 \%$ had corneal opacity and trichiasis or entropion. We labelled the latter category as "trachomatous" because trachoma is endemic in this area. However, leprosy itself can also cause trichiasis and entropion ${ }^{12}$; aetiology should be determined by examining for blepharochalasis (which may not be detectable at the time of examination), and for conjunctival scar. In this study, lids were not everted, so we may have overestimated (at leprosy's expense) the contribution made by trachoma to corneal opacification in these subjects. Based on funduscopic findings, glaucoma was identified as being responsible for only $6 \%$ of blindness. Glaucoma is thought to be uncommon in leprosy, perhaps because mean intraocular pressure is lower in those with leprosy than those without. ${ }^{13}$

Though completion of an appropriate course of anti-leprosy chemotherapy changes an individual's classification from "under active treatment" to "cured" in the registers of many leprosy control programmes, it does not prevent subsequent development of disabling complications, ${ }^{5}$ particularly those of the eye. ${ }^{12}{ }^{14}$ Nigeria's annual leprosy case detection rate in 2003 was $0.37 / 10000$ population, whereas its estimated nationwide leprosy prevalence is $0.45 / 10000$ (personal communication, Statistics Unit, National Tuberculosis and Leprosy Control Programme, Federal Ministry of Health, Abuja, Nigeria): most new patients successfully complete a course of MDT and are regarded as cured. Cured leprosy patients, however, will require ongoing ophthalmic care. There is an urgent need for better collaboration between leprosy control and blindness prevention programmes to enable this group to access high quality comprehensive eye care services. In the communities studied here, cataract surgery, early detection and management of lagophthalmos, and refractive services are all priorities. Additionally, a number of villages require community based trachoma control, with an emphasis on lid surgery. ${ }^{15}$ Reducing the incidence and prevalence of blindness and low vision in these patients will ease their suffering and increase their chance of social and economic rehabilitation.

\section{Authors' affiliations}

C Mpyet, Department of Ophthalmology, Jos University Teaching Hospital, PMB 2076, Jos, Nigeria

A W Solomon, International Centre for Eye Health, London School of Hygiene and Tropical Medicine, Keppel Street, London WCIE 7HT, UK

Funded by grants from Foundation Dark and Light Blind Care, Netherlands, and Netherlands Leprosy Relief.

Neither of the authors has any proprietary or commercial interest in the subject of the manuscript.

Correspondence to: Anthony W Solomon, PhD, International Centre for Eye Health, London School of Hygiene and Tropical Medicine, Keppel Street, London WCIE 7HT, UK; anthony.solomon@lshtm.ac.uk

Accepted for publication 9 August 2004

\section{REFERENCES}

1 Anon. Leprosy. Global situation. Wkly Epidemiol Rec 2002;77:1-8.

2 Anon. Progress towards the elimination of leprosy as a public health problem. Wkly Epidemiol Rec 1996;71:149-56.

3 Courtright $\mathbf{P}$, Lewallen S, Tungpakorn N, et al. Cataract in leprosy patients: cataract surgical coverage, barriers to acceptance of surgery, and outcome of surgery in a population based survey in Korea. $\mathrm{Br} J$ Ophthalmol 2001;85:643-7.

4 Smith CM, Smith WC. Leprosy at the cutting edge: 2000 to 2005, and beyond. Trop Doct 2002;32:46-8.

5 Courtright $P$, Lewallen S. Ocular manifestations of leprosy. In: Johnson GJ, Minassian DC, Weale RA, et al, eds. The epidemiology of eye disease. London: Arnold, 2003:306-17.

6 World Health Organization. WHO Study Group on the Prevention of Blindness. WHO Technical Report Series No 518. Geneva: WHO, 1973.

7 World Health Organization. A guide to leprosy control. 3rd ed. Geneva: WHO, 1988.

8 Schwab IR, Nassar E, Malaty R, et al. Leprosy in a trachomatous population. Arch Ophthalmol 1984;102:240-4.

9 Malu KN, Malu AO. Blindness in leprosy patients of Kaduna State, Northern Nigeria. Trop Doct 1995;25:181-3.

10 Nwosu SN, Nwosu MC. Ocular findings in leprosy patients in Nigeria. East Afr Med J 1994;71:441-4.

11 Hogeweg M. Cataract: the main cause of blindness in leprosy. Lepr Rev $2001 ; 72: 139-42$

12 Lewallen S, Tungpakorn NC, Kim SH, et al. Progression of eye disease in "cured" leprosy patients: implications for understanding the pathophysiology of ocular disease and for addressing eyecare needs. Br J Ophthalmol 2000;84:817-21

13 ffytche TJ. Leprosy and the eye. In: Rose FC, ed. The eye in general medicine. Baltimore: University Park Press, 1983:481.

14 Courtright $\mathrm{P}, \mathrm{Hu}$ LF, Li HY, et al. Multidrug therapy and eye disease in leprosy: a cross-sectional study in the People's Republic of China. Int J Epidemiol 1994;23:835-42.

15 Rabiu MM, Abiose A. Magnitude of trachoma and barriers to uptake of lid surgery in a rural community of northern Nigeria. Ophthalmic Epidemiol 2001;8:181-90. 


\section{PostScript}

\section{LETTERS}

If you have a burning desire to respond to a paper published in $B J O$, why not make use of our "rapid response" option?

Log onto our website (www.bjophthalmol. com), find the paper that interests you, and send your response via email by clicking on the "eletters" option in the box at the top right hand corner.

Providing it isn't libellous or obscene, it will be posted within seven days. You can retrieve it by clicking on "read eletters" on our homepage.

The editors will decide as before whether to also publish it in a futher paper issue.

\section{Subconjunctival corticosteroids for benign lymphoid hyperplasia}

Appropriate treatment of subconjunctival benign lymphoid hyperplasia (BLH) has been unclear. Most have noted poor response to oral or topical corticosteroids. Many recommend observation. Radiotherapy has been used, but there are risks of vision loss.

In this case, we found dramatic response to local subconjunctival injection of long acting corticosteroids, which may represent a therapeutic option for subconjunctival benign lymphoid hyperplasia.

\section{Case report}

A 72 year old woman noticed an enlarging mass on the nasal conjunctiva over the
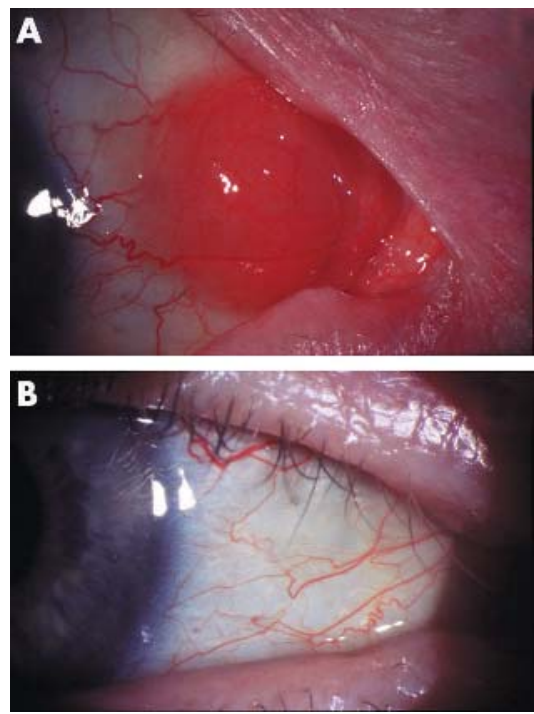

Figure 1 (A) Slit lamp photograph of the patient's right eye on initial presentation. Note the size, salmon colour and raised appearance of the lesion. (B) Slit lamp photograph of the same lesion 2 months later following subconjunctival injection of triamcinolone. The subconjunctival lesion appeared to have completely resolved with no obvious remnants seen on the sclera or conjunctiva. (Reproduced with permission.)

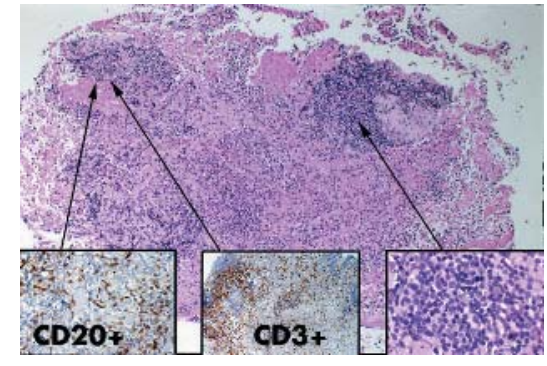

Figure 2 Haematoxylin and eosin staining, $10 \times$ magnification of the lesion biopsied in figure 1A. Note the abundance of lymphocytes seen more clearly in the magnified section in the lower right part of the figure. Also note the predominance of $\mathrm{T}$ cells (CD $3+$ ) with a less numerous population of $B$ cells (CD20+) typical of benign lymphoid hyperplasia.

previous year. She denied any discomfort, diplopia, or visual changes. She was pseudophakic in both eyes, and did not have any past ocular trauma, infection, or eye disease. She had a history of hypothyroidism, multiple myeloma, tuberculosis, and pulmonary sarcoidosis. Two months before presentation she was found to have colon carcinoma treated with colectomy and chemotherapy. She was reportedly free of any tumour metastasis.

Her visual acuity was 20/30 in the right eye and 20/40 in the left, and extraocular motility was full in both eyes. Examination revealed a salmon coloured, raised, and moderately firm patch on the nasal conjunctiva of the right eye without significant neovascularisation (fig 1A). Dilated fundus examination was unremarkable. A head MRI scan showed moderate enlargement of the medial rectus muscle without involvement of the muscle tendon. A simple biopsy $(2 \mathrm{~mm} \times 2 \mathrm{~mm} \times$ $1 \mathrm{~mm}$ ) of the lesion was performed.

Pathological examination revealed benign lymphohistiocytic infiltrates (fig 2). The lymphoid reaction showed a predominance of $\mathrm{T}$ cells $(\mathrm{CD} 3+)$, numerous histiocytes, and a smaller number of B cells (CD20+). There was no evidence of neoplastic plasma cells, metastatic carcinoma, or well defined sarcoid granulomas.

The patient elected to have surgical treatment over other options including observation. The patient received a $20 \mathrm{mg} / 0.5 \mathrm{ml}$ subconjunctival injection of triamcinolone (in $0.5 \mathrm{ml}$ in the nasal bulbar conjunctiva) just superior to the mass. On follow up examination 2 months later, the patient continued to deny any discomfort or visual changes and was very pleased about her response to the treatment. Remarkably, the lesion completely resolved being no longer visible or palpable (fig 1B). This patient unfortunately died 9 months later from complications secondary to a fall, but during this time there was no recurrence of the conjunctival lesion.

\section{Comment}

A patient presenting with a slow growing salmon coloured subconjunctival mass should always raise suspicion of neoplastic causes. Patients with ocular BLH and lymphoma will often have the similar presenting symptoms and demographic profiles. In addition they appear very similar radiologically, ${ }^{1}$ and thus definitive diagnosis requires tissue biopsy. A pathological diagnosis of BLH traditionally requires reactive follicles, polyclonality, and the absence of cytological atypia. $^{2}$ Lymphoproliferative lesions can occur throughout the ocular adnexa, and some studies suggest a more benign course for conjunctival BLH compared to those in the orbit. ${ }^{3}$ Coupland et al found that of 112 cases, $32(29 \%)$ were in the conjunctiva, 52 $(46 \%)$ in the orbit and the remainder in the eyelid, lacrimal gland, and caruncle. ${ }^{4}$ The optimal treatment for BLH is uncertain. Many recommend frequent observation. Others have tried focal radiotherapy with some success, ${ }^{5}$ but there is a significant risk of vision loss. ${ }^{6}$ In a recent review of 117 cases of conjunctival lymphoproliferative lesions, $17 \%$ were BLH, 22\% were atypical lymphoid hyperplasia, and 56\% were lymphoma. ${ }^{7}$ In these cases 9\% were observed, $42 \%$ had complete excisional biopsy, $4 \%$ had biopsy and cryotherapy, $44 \%$ had biopsy and external beam irradiation, and 6\% had biopsy and chemotherapy. $^{7}$

In this case report, we found a dramatic response to local subconjunctival injection of a long acting corticosteroid. The corticosteroid near the reactive follicle must have been sufficient to suppress lymphocyte proliferation. This response may represent a therapeutic option for BLH.

D G Telander, T Z Lee, S E Pambuccian, A J W Huang

Department of Ophthalmology, University of Minnesota, Minneapolis, MN, USA

S E Pambuccian

Department of Pathology, University of Minnesota, Minneapolis, MN, USA

D G Telander

UCLA/Jules Stein Eye Institute, Los Angeles, CA, USA

T Z Lee

Apple Hill Eye Center, 25 Monument Road, Suite 297, York, PA 17403, USÁ

Correspondence to: Andrew J W Huang, MD, Department of Ophthalmology, University of Minnesota, 516 Delaware Street SE, 9th Floor, Minneapolis, MN 55455, USA; huang088@umn.edu'

doi: 10.1136/bjo.2004.051342

Accepted for publication 1 October 2004

\section{References}

1 Knowles DM, Jakobeic FA. Malignant lymphoma and lymphoid hyperplasia occurring in the ocular adnexa. In: Knowles DA, ed. Neoplastic hematology. Baltimore: Williams \& Wilkins, 1992:1009-46.

2 Cockerham GC, Jakobiek FA. Lymphoproliferative disorders of the ocular adnexa. Int Ophthalmol Clin 1997;37:39-59.

3 Knowles DM, Jakobiek FA, McNally L, et al. Lymphoid lyperplasia and malignant lymphoma occurring in the ocular adnexa (orbit, conjunctiva, and eyelids): a prospective multiparametric analysis of 108 cases during 1977 to 1987 . Hum Pathol 1990;21:959-73. 
4 Coupland SE, Krause L, Delecluse H-J, et al. Lymphoproliferative lesions of the ocular adenexa. Analysis of 112 cases. Ophthalmology 1998:105:1430-41.

5 Keleti D, Flickinger JC, Hobson SR, et al. Radiotherapy of lymphoproliferative diseases of the orbit. Surveillance of 65 cases. Am J Clin Oncol 1992;15:422-7

6 Bessel EM, Henk JM, Whitelocke RAF, et al. Ocular morbidity after radiotherapy of orbital and conjunctival lymphoma. Eye 1987;1:90-96.

7 Shields CL, Shields JA, Carvalho C, et al. Conjunctival lymphoid tumors. clinical analysis of 117 cases and relationship to systemic lymphoma. Ophthalmology 2001;108:979-84.

\section{Congenital upper eyelid eversion complicated by corneal perforation}

Congenital upper eyelid eversion is a rare condition more frequently seen in black infants and in Down's syndrome. If recognised early, the condition can be managed conservatively without recourse to surgery. We highlight a case that presented late with severe sight threatening complications. Surgical intervention was consequently the only appropriate way to manage the patient.

\section{Case report}

A 7 month old black female was referred to the emergency room of the King Khaled Eye Specialist Hospital in Riyadh, Saudi Arabia. She had a history of a white spot in the right cornea, and a right upper lid that had flipped up since birth.

She was born by breech delivery and was subsequently diagnosed as having Down's syndrome. On examination she had complete eversion of the right upper lid with mild conjunctival chemosis. The lid could be repositioned with ease but when the child cried it reverted. There was a corneal opacity with a central descematocele and iris adhesion to the endothelium. The child was admitted and started on intensive topical antibiotics. In spite of manual inversion of the upper lid as well as a moist chamber, the lid continued to re-evert. A lateral tarsorrhaphy and penetrating keratoplasty were performed under general anesthesia but postoperatively, the eyelid continued to evert (fig l). Because of the risk of exposure to the transplanted cornea, a full thickness skin graft to the right upper lid was performed (fig 2). Subsequently, the lid retained its normal position and the child could fix and follow adequately with the eye. The transplanted cornea was clear at the 10 week postoperative visit after which the patient was lost to follow up.

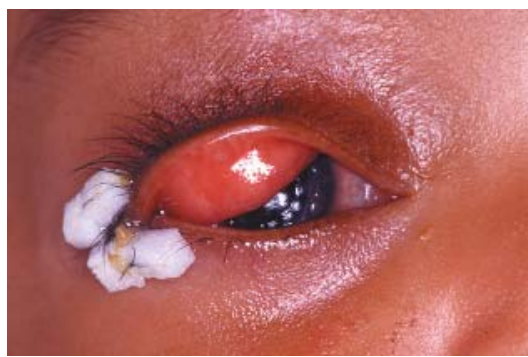

Figure 1 After the tectonic penetrating keratoplasty was performed, the lateral temporal tarsorrhaphy failed to retain the lid in an inverted position.

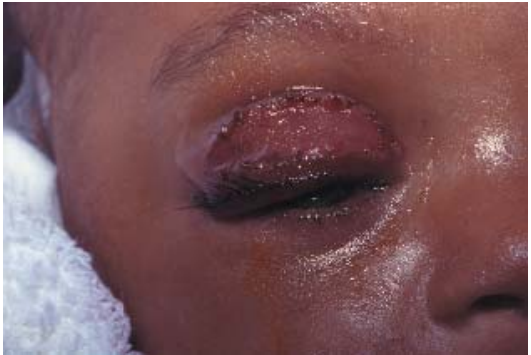

Figure 2 Full thickness skin graft to the upper lid successfully expanded the anterior lamella to permanently solve the problem.

\section{Comment}

Congenital eversion of the upper eyelid was first described in 1896 by Adams ${ }^{1}$ who called the condition "double congenital ectropion." The exact incidence of this condition is not known. Sellar reviewed the literature in 1992 and found 51 reported cases. Since then only two more case reports could be found in the literature. ${ }^{34}$ The eversion is usually present at birth, but late onset of total eversion of the upper eyelids has been described in infancy, ${ }^{5}$ and as late as 11 years of age.

The condition typically is bilateral and asymmetrical but unilateral cases have been described. The underlying pathophysiology is obscure and several possible mechanisms have been proposed and associations recognised. The incidence appears to be higher in black infants, ${ }^{6}$ infants with trisomy $21,{ }^{2}$ and in infants born with collodion skin disease. Abnormalities such as orbicularis hypotonia, ${ }^{8}$ birth trauma, vertical shortening of the anterior lamellar or vertical elongation of the posterior lamellar of the eyelid and failure of the orbital septum to fuse with the levator aponeurosis (with adipose tissue interposition), ${ }^{9}$ absence of effective lateral canthal ligament, and lateral elongation of the eyelid have all been implicated as possible pathophysiological factors. Once everted, orbicularis spasm $^{10}$ may act as a sphincter that leads to a vicious cycle of conjunctival strangulation and oedema secondary to venous stasis. This chemotic conjunctiva usually protects the cornea from exposure and, hence, corneal complications have thus far not been reported in infants. To our knowledge this is the first reported case of congenital eyelid eversion complicated by corneal exposure, descematocele, and perforation.

Congenital eyelid eversion either resolves spontaneously with conservative treatment or with surgical intervention such as a subconjunctival injection of hyaluronic acid, tarsorrhaphy with excision of redundant conjunctiva, fornix sutures, and full thickness skingraft to the upperlid. In this patient because of failure of all conservative measures, the chronicity ( 7 months) and severity (corneal ulcer/perforation), we elected to do anterior lamellar lengthening by means of a full thickness upper lid skin graft. Corneal perforation necessitating penetrating keratoplasty is a rare complication of congenital eyelid eversion, but in this case it was essential to maintain the integrity of the globe and to, hopefully, retain vision and prevent deep amblyopia.

The condition in this reported case of congenital eyelid eversion complicated by a corneal ulcer, large descematocele and perforation, is rare. Congenital upper lid eversion may present "once in a lifetime" to the ophthalmologist or newborn nursery paediatrician. It is nevertheless important to create awareness among healthcare professionals in obstetric and neonatal care of the existence of this potentially sight threatening congenital anomaly, as the condition is very amenable to early treatment.

H Al-Hussain, A A Al-Rajhi, S Al-Qahtani, D Meyer

University of Stellenbosch/King Khaled Eye Specialist Hospital, Faculty of Health Sciences, PO Box 19059, Tygerberg, South Africa

Correspondence to: Professor David Meyer, University of Stellenbosch, Faculty of Health Sciences, PO Box 19059, Tygerberg, South Africa; dm2@sun.ac.za doi: 10.1136/bjo. 2004.053348

Accepted for publication 20 October 2004

\section{References}

1 Adams AL. A case of double congenital ectropion. Med Fortnightly 1896;9:137-8.

2 Sellar PW, Bryars JH, Archer DB. Late presentation of congenital ectropion of the eyelids in a child with Down's syndrome: a case report and review of the literature. J Pediatr Ophthalmol Strabismus 1992;29:64-7.

3 Watts MT, Dapling RB. Congenital eversion of the upper eyelid: a case report. Ophthal Plast Reconstr Surg 1995;11:293-5.

4 Dawodu OA. Total eversion of the upper eyelids in a newborn. Niger Postgrad Med J $2001 ; 8: 145-7$

5 Siverstone B, Hirsch I, Sternberg MD, et al. Late onset of total eversion of upper eyelids. Ann Ophthalmol 1982;14:477-8.

6 Lu LW, Bansal RK, Katzman B. Primary congenital eversion of the upper lids. J Pediatr Ophthalmol Strabismus 1979:16:149-51.

7 Shapiro RD, Soentgen ML. Collodion skin disease and everted eyelids. Postgrad Med 1969;45:216-19.

8 Loeffler M, Hornblass A. Surgical management of congenital upper-eyelid eversion. Ophthalmic Surg 1990;21:736.

9 Blechman B, Isenberg S. An anatomical etiology of congenital eyelid eversion. Ophthalmic Surg 1984;15:111-13.

10 Raab EL, Saphir RL. Congenital eyelid eversion with orbicularis spasm. J Pediatr Ophthal Strabismus 1985;22:125-8.

\section{Homozygous mutation (L527R) of $T G F B I$ in an individual with lattice corneal dystrophy}

Lattice corneal dystrophy (LCD), an inherited form of amyloidosis, is characterised by the development of lattice lines and opacity in the cornea. LCD is classified clinically into four subtypes: I, II, III, and IIIA. Several distinct mutations of TGFBI have been associated with LCDIIIA: P501T, ${ }^{1}$ L527R, ${ }^{2}$ N544S, ${ }^{3}$ A546T, ${ }^{4}$ N622K (T1913G and T1913A), and V627S. ${ }^{5}$ All cases of LCD characterised at the molecular genetic level to date have been attributed to heterozygous point mutations of TGFBI. We now present the first example of a homozygous point mutation of TGFBI in an individual with LCD, a diagnosis supported by clinical, histological, and molecular genetic findings.

\section{Case report}

A 52 year old Japanese man visited our corneal clinic in July 1997 with a main complaint of gradual impairment of vision. His parents, who were related, were no longer alive and he had no children. He had two brothers and four sisters. His reporting 


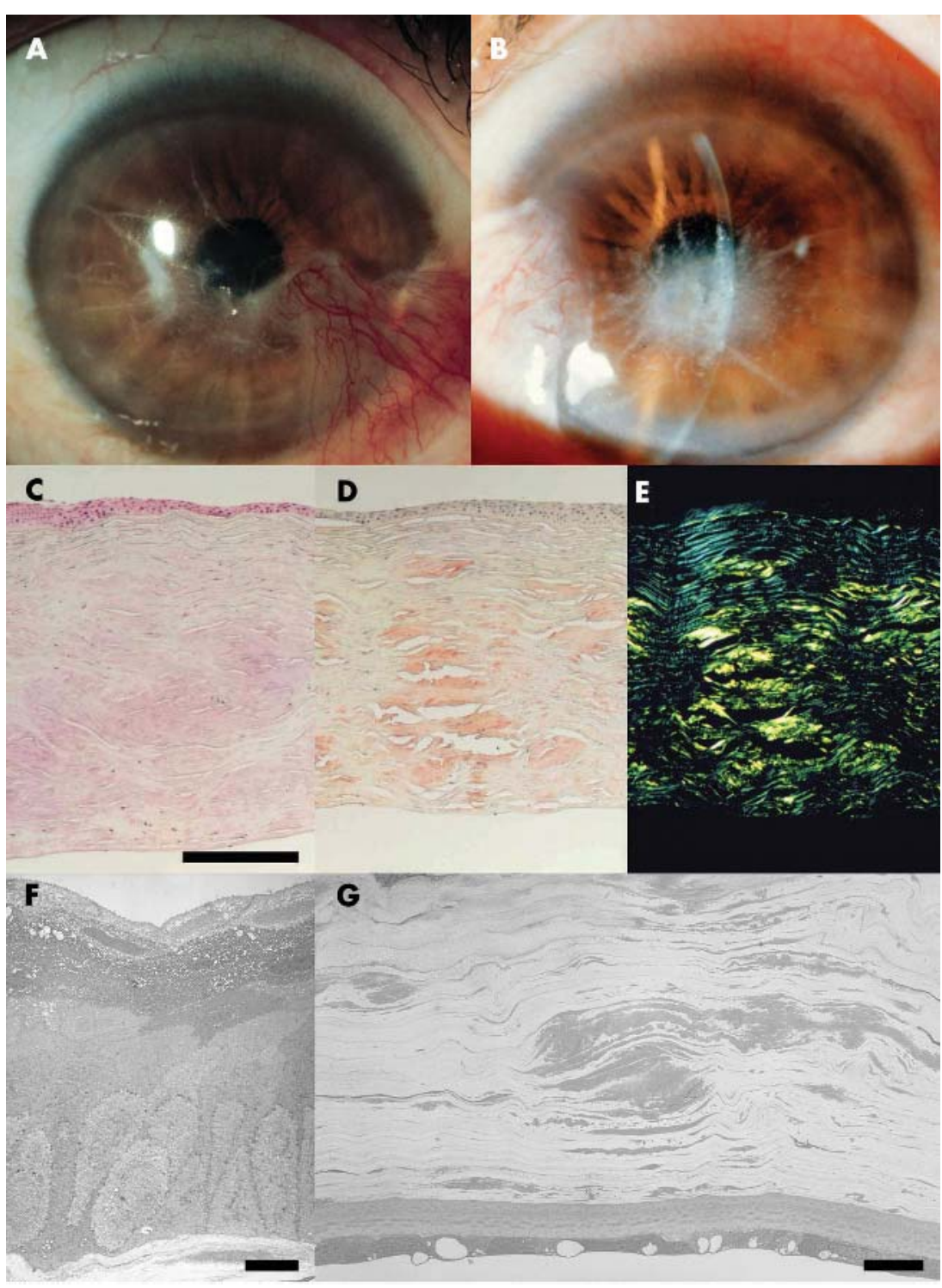

Figure 1 (A and B) Slit lamp photographs of the right and left eyes, respectively, of the patient. Light microscopic (C-E) and transmission electron microscopy (TEM) (F and G) analysis of the surgically removed corneal specimens of the patient. (C) Haematoxylin and eosin staining of the left cornea. (D) Congo red staining of the left cornea. (E) Polarised light microscopy of the left cornea. (F) TEM of the epithelial cell layer of the right cornea. (G) TEM of the stromal and endothelial cell layers of the right cornea. Scale bars: $250 \mu \mathrm{m}(\mathrm{C}-\mathrm{E})$ or $10 \mu \mathrm{m}$ (F and G).

suggested that his father had had LCD and that his younger sister had also developed this condition.

Slit lamp examination revealed a large region of opacity and thick lattice lines in the middle to deep portion of the corneal stroma in both eyes (fig IA and B). Given that his visual acuity in both eyes decreased to $20 / 40$, we performed penetrating keratoplasty on his left eye in December 2000 and on his right eye in May 2002. After the surgeries, his visual acuity improved to 20/25 in each eye and no recurrence has been observed to date.

Histological analysis revealed that the amorphous component of the middle to deep region of the stroma of both corneas stained with eosin (fig lC) and with Congo red (fig 1D). The detection of apple green dichroism by polarised light microscopy was also consistent with amyloid deposition in the middle to deep region of the corneal stroma (fig 1E). Although most of the epithelial cell layer, basement membrane, and hemidesmosomes of the right cornea appeared normal by transmission electron microscopy (TEM) (fig lF), a region was detected that seemed to be devoid of basal cells, basement membrane, and hemidesmosomes. TEM also revealed amyloid deposits in the middle to deep corneal stroma (fig lG), although Descemet's membrane and endothelial cells appeared normal.

After obtaining informed consent, we purified DNA from the white blood cells isolated from $10 \mathrm{ml}$ of the patient's blood. With appropriate primers, ${ }^{6}$ we amplified exons 4 and 12 of TGFBI by the polymerase chain reaction (PCR) and directly sequenced the products. We detected a homozygous point mutation, CTG $\rightarrow$ CGG (L527R), in codon 527 of TGFBI in the proband (fig 2). Point mutations were not detected in codons $124,518,544,546$, or 555 , mutations in

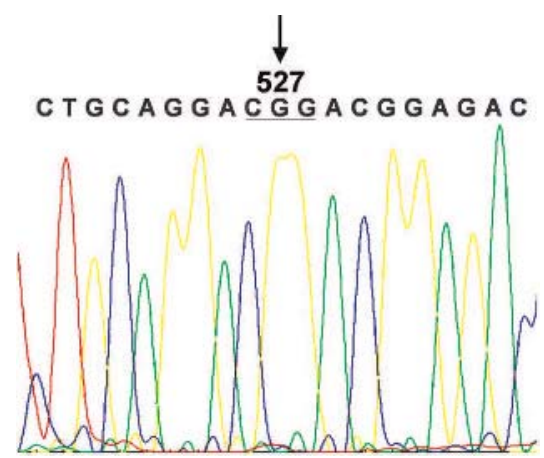

Figure 2 Molecular genetic analysis of TGFBI of the patient. Direct sequencing of PCR products corresponding to exon 12 of TGFBI. A homozygous $T \rightarrow G$ mutation (arrow) was detected at codon 527.

which have been associated with corneal dystrophies.

\section{Comment}

Fujiki $e$ e $a l^{2}$ reported that LCDIIIA in L527R heterozygotes is characterised by a late onset and mild clinical findings. Hirano et $a l^{7}$ reported that the condition caused by L527R heterozygosity was associated with amyloid deposition in the deep corneal stroma but not with corneal erosion. In contrast, LCD in our patient with a homozygous L527R mutation was characterised by onset in middle age, recurrent corneal erosion, and amyloid deposition in the middle to deep region of the stroma. The deposits in the proband showed right eye-left eye asymmetry in size and shape, as previously described for some L527R heterozygotes. ${ }^{2}$

In general, corneal dystrophies caused by homozygous point mutations in TGFBI are characterised by an earlier onset, more severe symptoms, and a higher frequency of recurrence after keratoplasty compared with those attributable to the corresponding heterozygous mutations. ${ }^{8-12}$ Our study and the two previous studies $^{27}$ of this mutation suggest that this is also the case for L527R. However, the difference in the findings of slit lamp examination between L527R heterozygotes and homozygotes with LCD appears to be less marked than that observed between R124H heterozygotes and homozygotes with Avellino corneal dystrophy. The reason for this discrepancy remains unclear at present.

\section{Acknowledgements}

This research was supported by grants from the Japan Society for the Promotion of Science (JSPS) and the Ministry of Education, Culture, Sports, Science, and Technology of Japan (to MI and TN).

\section{N Yamada, T-i Chikama, N Morishige,} R Yanai, T Nishida

Department of Biomolecular Recognition and Ophthalmology, Yamaguchi University School of Medicine, Ube City, Yamaguchi 755-8505, Japan

N Yamada, R Yanai, M Inui

Department of Pharmacology, Yamaguchi University School of Medicine, Ube City, Yamaguchi 755-8505, Japan

K Seki

Department of Ocular Pathophysiology, Yamaguchi University School of Medicine, Ube City, Yamaguchi 755-8505, Japan 
Correspondence to: Naoyuki Yamada, MD, PhD Department of Biomolecular Recognition and Ophthalmology, Yamaguchi University School of Medicine, 1-1-1 Minami Kogushi, Ube City, Yamaguchi 755-8505, Japan n.yamada@po.cc.yamaguchi-u.ac.jp

doi: $10.1136 /$ bjo. 2004.056168

Accepted for publication 1 November 2004

\section{References}

Yamamoto S, Okada M, Tsujikawa M, et al. A kerato-epithelin (betaig-h3) mutation in lattice corneal dystrophy type IIIA. Am J Hum Genet 1998:62:719-22.

2 Fujiki K, Hotta Y, Nakayasu K, et al. A new L527R mutation of the betalGH3 gene in patients with lattice corneal dystrophy with deep stromal opacities. Hum Genet 1998;103:286-9.

3 Mashima $Y$, Yamamoto $S$, Inove $Y$, et al. Association of autosomal dominantly inherited corneal dystrophies with BIGH3 gene mutations in Japan. Am J Ophthalmol 2000;130:516-17.

4 Dighiero $P$, Drunat $S$, Ellies $P$, et al. A new mutation (A 546T) of the betaig-h3 gene responsible for a French lattice corneal dystrophy type IIIA. Am J Ophthalmol 2000;129:248-51.

5 Munier FL, Frueh BE, Othenin-Girard $\mathrm{P}$, et al. $\mathrm{BIGH} 3$ mutation spectrum in corneal dystrophies. Invest Ophthalmol Vis Sci 2002;43:949-54.

6 Munier FL, Korvatska E, Djemai A, et al. Keratoepithelin mutations in four 5q31-linked corneal dystrophies. Nat Genet 1997;15:247-51.

7 Hirano K, Hotta Y, Nakamura M, et al. Late-onse form of lattice corneal dystrophy caused by Leu527Arg mutation of the TGFBI gene. Cornea 2001;20:525-9.

8 Mashima $Y$, Konishi M, Nakamura $Y$, et al. Severe form of juvenile corneal stromal dystrophy with homozygous $\mathrm{R} 124 \mathrm{H}$ mutation in the keratoepithelin gene in five Japanese patients. Br J Ophthalmol 1998;82:1280-4.

9 Okada M, Yamamoto S, Watanabe $\mathrm{H}$, et al. Granular corneal dystrophy with homozygous mutations in the kerato-epithelin gene. Am J Ophthalmol 1998;126:169-76.

10 Okada M, Yamamoto S, Inove Y, et al. Severe corneal dystrophy phenotype caused by homozygous R124H keratoepithelin mutations. Invest Ophthalmol Vis Sci 1998;39:1947-53.

11 Fujiki K, Hotta Y, Nakayasu K, et al. Homozygotic patient with betaig-h3 gene mutation in granular dystrophy. Cornea 1998;17:288-92.

12 Inove T, Watanabe H, Yamamoto S, et al. Different recurrence patterns after phototherapeutic keratectomy in the corneal dystrophy resulting from homozygous and heterozygous $\mathrm{R} 124 \mathrm{H}$ BIG-H3 mutation. Am J Ophthalmol 2001;132:255-7.

\section{Vitreous amyloidosis in alanine 71 transthyretin mutation}

Familial amyloid polyneuropathy (FAP) associated with mutations in the transthyretin (TTR) gene is the commonest form of hereditary amyloidosis. The incidence of vitreous opacities in FAP varies from 5.4\% to $35 \%,{ }^{12}$ but vitreous opacities as part of systemic amyloidosis are virtually pathognomonic of FAP. Hereditary non-neuropathic systemic amyloidosis is associated with mutations in the genes for lysosyme, apolipoprotein A-I, or fibrinogen A $\alpha$-chain. There are some 80 known mutations in TTR gene of which the methionine 30 variant is the most common. ${ }^{3}$ The rare alanine 71 (Ala 71) variant with vitreous opacities has been described in one family from France and another from Spain. ${ }^{45}$ We report a case of FAP Ala 71 without a family history of the disease who presented with a monocular inferior visual field defect and a corresponding vitreous opacity. Amyloid deposition was
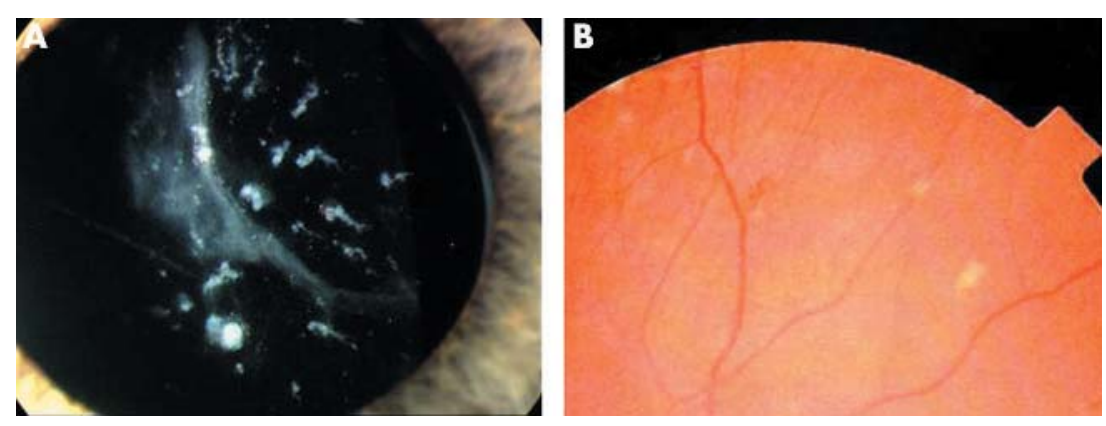

Figure 1 Dense nodular white deposits on the posterior capsule of the left eye were presumed to be of amyloid origin (A), and fundus periphery demonstrates intraretinal haemorrhages and perivascular white deposits (B)

subsequently diagnosed on vitreous and sural nerve biopsy.

\section{Case report}

A 46 year old woman presented with an inferior visual defect in her left eye. Ocular and systemic evaluation was normal including brain computed tomography scan. Eighteen months later, the patient developed bilateral floaters and visual loss in the left eye reducing her vision to 20/15 right and 20/30 left. Deposits of white "fluffy" material were noted on the posterior capsule of the left eye (fig lA) as well as bilateral "branching" vitreous opacities, peripheral retinal haemorrhages and perivascular sheathing (fig 1B). The left eye had a partial posterior vitreous detachment (PVD), a large vitreous floater and old inferior vitreous haemorrhage $(\mathrm{VH})$.

Vitreous opacification progressed and visual acuity was reduced to hand movement l year later. A full blood count, coagulation screen, and biochemical profile were normal. Creatinine clearance demonstrated a mild reduction in renal function and plasma cell dyscrasia was ruled out. Pars plana vitrectomy successfully cleared the vitreous debris, restoring vision to $20 / 20$ in the left eye. Eighteen months later the patient developed a right foot drop, progressive lower limb numbness, and numbness in both hands. A sural nerve biopsy established the diagnosis of amyloidosis and immunohistochemistry confirmed that TTR was the major protein constituent of the deposits. Cardiac involvement was demonstrated on echocardiography and renal involvement was confirmed by serum amyloid $\mathrm{P}$ (SAP) scintigraphy. Sequencing of her TTR gene confirmed that she was heterozygous for the amyloidogenic Ala 71 variant.

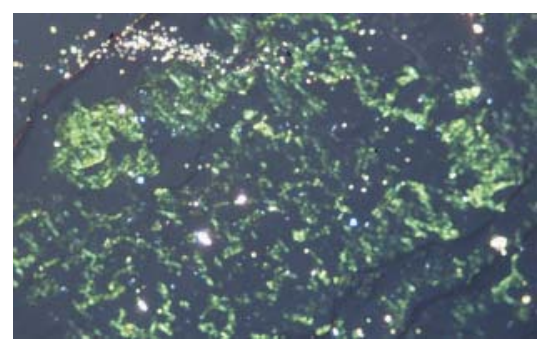

Figure 2 Apple-green birefringence with Congo red stain viewed with polarised microscopy of a vitreous biopsy confirming the presence of amyloid deposits.
The patient's right visual acuity deteriorated to $20 / 120$ because of increasing white vitreous opacities and nodular opacities on the anterior vitreous face. Right vitrectomy resulted in a return of visual acuity to 20/20. An undiluted right vitreous biopsy confirmed large amounts of amyloid of the TTR type (fig 2). The patient currently awaits orthoptic liver transplantation (OLT).

\section{Comment}

Vitreous opacification was initially attributed to old VH secondary to idiopathic retina vasculitis. Vitreous biopsy subsequently confirmed amyloid in the fellow eye. VH may occur secondary to vascular adventitial amyloid deposition or vitreous separation leading to a retinal tear, although vitreous opacities may be misinterpreted as Kantarjian and de Jong first reported vitreous amyloid in FAP. Amyloid of the vitreous body has been described as "glass wool, sheet-like veils or string of pearls white opacities," which differs from localised ocular amyloid in the orbit, lacrimal gland, conjunctiva, eyelids, sclera, and more specific forms in the cornea. Previous studies have reported abnormal conjunctival vessels, pupillary abnormalities, keratoconjunctivitis sicca, glaucoma, and vitreous opacities.

Almost all of the circulating TTR is produced in the liver and OLT can halt the progression of this disease and lead to clinical improvement. ${ }^{7}$ SAP scintigraphy is a method for identifying and quantitatively monitoring amyloid deposits in vivo, ${ }^{7}$ but this technique is not sensitive enough to monitor vitreous amyloid. Surprisingly, progressive vitreous amyloid deposition has been reported follow ing OLT, suggesting the TTR that forms vitreous amyloid may be produced locally. ${ }^{8}$ Previous reports have established that TTR has a widespread distribution in the eye but TTR mRNA has exclusively been located in the retinal pigment epithelium (RPE). ${ }^{9}$ Given that plasma TTR does not cross Bruch's membrane, it appears that ocular TTR is synthesised at least in part in the RPE, but the exact factors determining amyloid deposition are not understood.

Bilateral "branching" vitreous deposits of unknown aetiology should always raise the possibility of systemic amyloidosis. A relevan family history should be sought, but even in its absence, mutations of the TTR gene should be looked for.

H J Zambarakii, D G Charteris Moorfields Eye Hospital, Vitreoretinal Service London, UK 
W Ayliffe

Mayday University Hospital, Ophthalmology Department, London, UK

P J Luthert

Institute of Ophthalmology, Pathology Department London, UK

F Schon

Mayday University Hospital, Neurology Department London, UK

P N Hawkins

National Amyloidosis Centre, Department of Medicine, Royal Free and University College Medical School, London, UK

Correspondence to: $\mathrm{Dr} \mathrm{H}$ Zambarakji, Angiogenesis, Massachusetts Eye and Ear Infirmary, 325 Cambridge Street, Boston, MA 02114, USA hzambaraji@aol.com or

HZ@meei.harvard.edu

doi: 10.1136/bjo.2004.057554

Accepted for publication 20 October 2004

\section{References}

1 Ando E, Ando Y, Okamura R, et al. Ocular manifestations of familial amyloidotic polyneuropathy type I: long term follow up. Br J Ophthalmol 1997;81:295-8.

2 Koga T, Ando E, Hirata A, et al. Vitreous opacities and outcome of vitreous surgery in patients with familial amyloidotic polyneuropathy. Am J Ophthalmol 2003;135:188-93.

3 Connors LH, Richardson AM, Theberge R, et al. Tabulation of transthyretin (TTR) variants as of $1 /$ 1/2000. Amyloid 2000;7:54-69.

4 Benson MD, Turpin JC, Lucotte G, et al. A transthyretin variant (alanine 71) associated with familial amyloidotic polyneuropathy in a French family. J Med Genet 1993;30:120-2.

5 Almeida MR, Lopez-Andreu F, Munar-Ques M, et al. Transthyretin ALA 71: a new transthyretin variant in a Spanish family with familial amyloidotic polyneuropathy. Hum Mutat 1993:2:420-1.

6 Kantariian AD, de Jong RN. Familial primary amyloidosis with nervous system involvement. Neurology 1953;3:399-409.

7 Rydh A, Suhr O, Hietala SO, et al. Serum amyloid $\mathrm{P}$ component scintigraphy in familial amyloid polyneuropathy: regression of visceral amyloid following liver transplantation. Eur J Nuc Med 1998;25:709-13.

8 Munar-Ques M, Salva-Ladaria L, Mulet-Perera P, et al. Vitreous amyloidosis after liver transplantation in patients with familial amyloid polyneuropathy: ocular synthesis of mutant transthyretin. Amyloid 2000;7:266-9.

9 Cavallaro T, Martone RL, Dwork AJ, et al. The retinal pigment epithelium is the unique site of transthyretin synthesis in the rat eye. Invest Ophthalmol Vis Sci 1990;31:497-501.

\section{Multifocal electroretinogram demonstrated macular toxicity associated with ethambutol related optic neuropathy}

Ethambutol is an effective drug in the first line treatment for tuberculosis but its use may be associated with ocular toxicity. ${ }^{1}$ Toxic optic neuropathy is the most important ocular side effect and is related to the dose and duration of treatment. ${ }^{2}$ It is usually bilateral and both central and peripheral types of optic neuropathy have been described. The central type involves the papillomacular bundle and results in decreased visual acuity, caecocentral scotoma, and blue-yellow colour vision loss, whereas the peripheral type causes peripheral visual field loss, especially bitemporal defects with sparing of visual acuity and red-green colour vision impairment. ${ }^{3}$ In additional to the optic nerve toxicity, studies have also demonstrated that ethambutol may also be toxic at the retinal level. ${ }^{4-6}$ We report a patient with ethambutol related toxic optic neuropathy associated with bilateral macular toxicity as demonstrated by multifocal electroretinogram (mfERG). To our knowledge, evaluation of ethambutol related macular toxicity with mfERG has not been previously reported

\section{Case report}

A 45 year old man with pulmonary tuberculosis presented with a 3 week history of gradual bilateral visual loss. He has been on antituberculosis therapy with ethambutol $900 \mathrm{mg}$ (15 mg/kg/day), rifampicin $600 \mathrm{mg}$, isoniazid $300 \mathrm{mg}$, and pyrazinamide $2 \mathrm{~g}$ for 4 months. The baseline best corrected visual acuity (BCVA) before antituberculosis therapy was 20/30 in the right eye and 20/20 in the left eye. On presentation, the BCVA was 20/200 bilaterally without afferent pupillary defect. Colour vision testing showed red green dyschromatopsia. Dilated fundus examination revealed bilateral small splinter haemorrhages adjacent to the optic discs with mild left optic disc swelling (fig lA). Visual field examination showed bilateral central scotoma (fig lB). Magnetic resonance images (MRI) of the brain and orbits were normal. A diagnosis of toxic optic neuropathy due to ethambutol was made.

Since the optic discs and fundus changes were relatively subtle compared with the reduction in visual acuity and the central visual field defects and red-green dyschromatopsia were atypical, further investigations

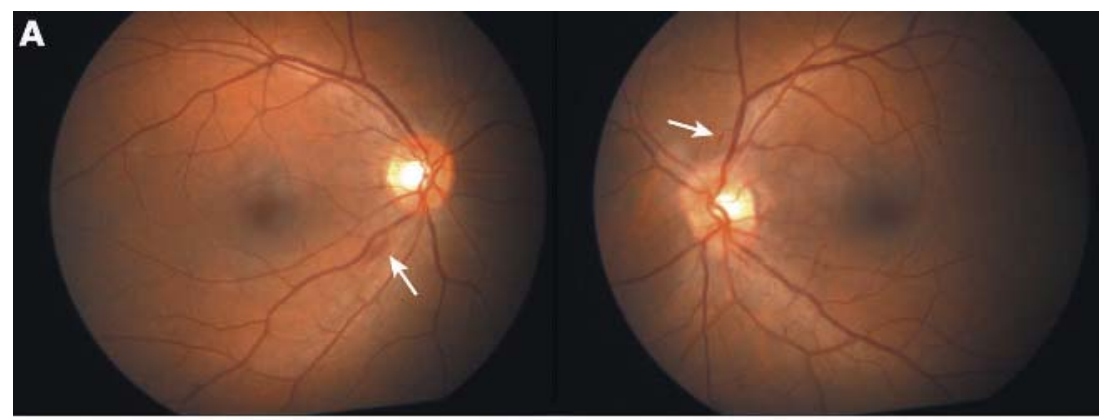

$\mathbf{B}$
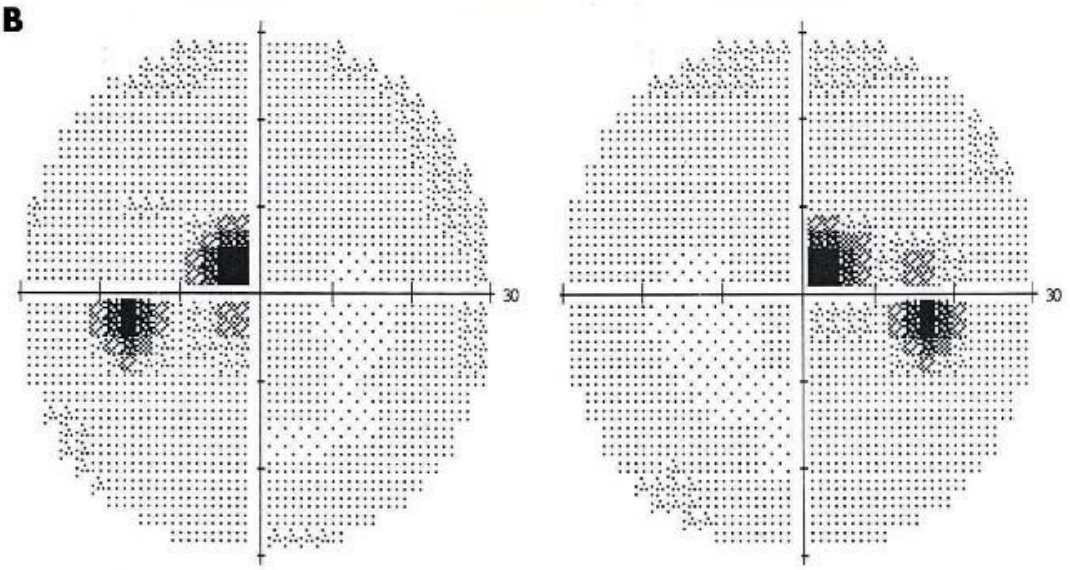

Figure 1 (A) Fundus photograph of the right (left) and left (right) eyes on presentation demonstrating bilateral splinter haemorrhages (arrows) with mild left optic disc swelling. (B) Automated visual field test showed central scotoma in the left (left) and right (right) eyes. were performed to evaluate potential associated retinal toxicity. These included electrooculogram (EOG), flash electroretinogram (ERG), pattern visual evoked potential (VEP), and mfERG. EOG and flash ERG of both eyes were normal. Pattern VEP showed and response for smaller checkers, with weak $1.8^{\circ}$. MfERG demonstrated bilateral general ised reduction in $\mathrm{Nl}$ and $\mathrm{Pl}$ retinal response amplitudes suggestive of toxic maculopathy (fig 2A).

In view of the optic neuropathy and maculopathy, all antituberculosis drugs were stopped after discussion with the physicians. The patient's BCVA gradually improved and 3 months later, his BCVA improved to 20/30 bilaterally. No abnormality was seen on fundus examination. Repeat mfERG recording showed recovery of the retinal responses in both eyes (fig 2B).

\section{Comment}

The exact mechanism of ethambutol induced toxic optic neuropathy is unclear but it may be due to retinal ganglion cells or bipolar cells toxicity at the retinal level. ${ }^{4-7}$ mfERG is a technique that allows objective assessment of (n) diseases ${ }^{8}$. In ERG which stimulates and measures the response of the entire retina, mfERG stimulates individual macular areas and measures responses from different retinal locations. It is useful in differentiating macular and optic nerve diseases as the mfERG in patients with optic nerve disease should be normal. ${ }^{9}{ }^{10}$ With the use of mfERG, we demonstrated that there was generalised reduction in MfERG responses at the macula and the areas of 

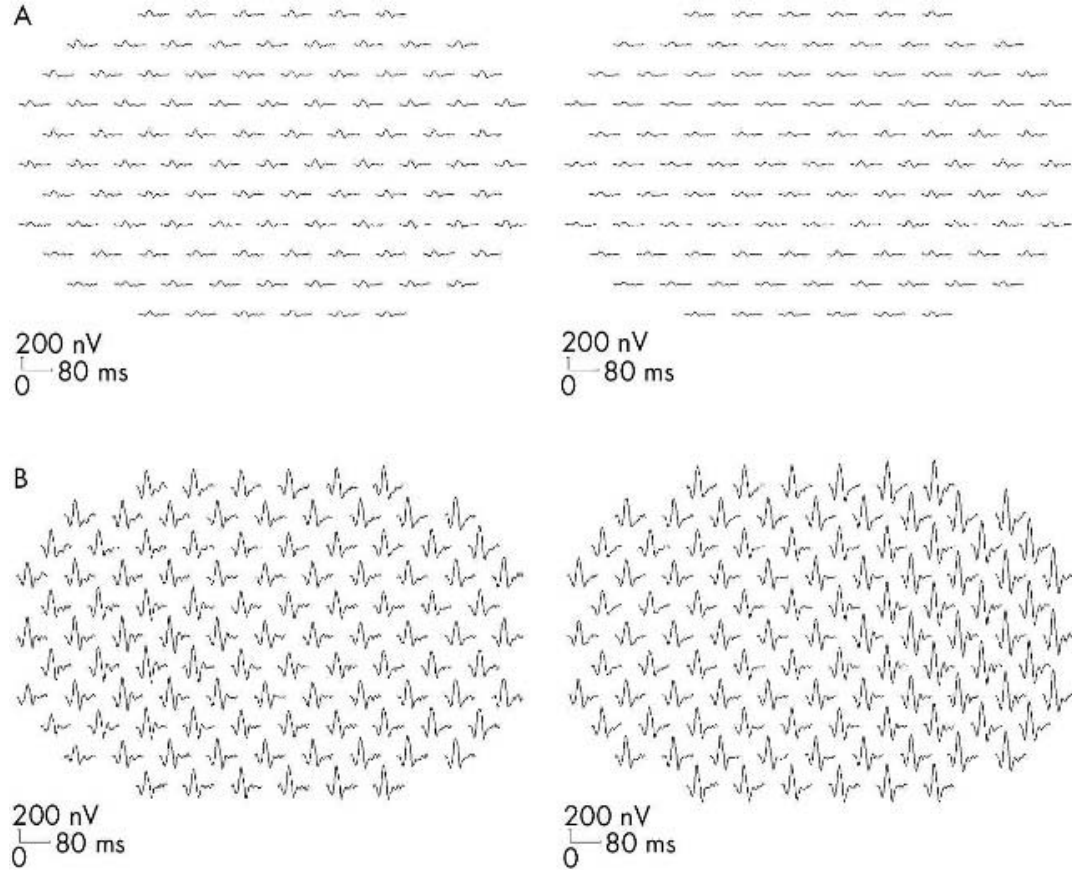

Figure 2 (A) Trace arrays of multifocal electroretinogram (mfERG) of the right (left) and left (right) eyes on presentation showing generalised reduction of retinal responses at the macula. (B) Trace arrays of mfERG of the right (left) and left (right) eyes 3 months after cessation of ethambutol demonstrating recovery and improvement of the $\mathrm{mfERG}$ responses.

abnormality were more extensive than the apparent localised visual field defects detected by automated perimetry. The abnormal mfERG suggests that in addition to toxic optic neuropathy, ethambutol may also cause macular toxicity at the neurosensory retinal level. The improvement in the patient's BCVA paralleled the improvement in mfERG responses and therefore mfERG may be a useful tool not only in diagnosis but in the serial assessments of ethambutol related ocular toxicity, particularly in patients with central visual loss.

T Y Y Lai, W-M Chan, D S C Lam Department of Ophthalmology and Visual Sciences, The Chinese University of Hong Kong, Hong Kong Eye Hospital, Hong Kong, People's Republic of China

E Lim

Department of Ophthalmology and Visual Sciences, The Chinese University of Hong Kong, Prince of Wales Hospital, Hong Kong, People's Republic of China

Correspondence to: Dr Wai-Man Chan, Department of Ophthalmology and Visual Sciences, The Chinese University of Hong Kong, 3/F, Hong Kong Eye Hospital, 147K Argyle Street, Kowloon, Hong Kong; cwm6373@netvigator.com

doi: 10.1136/bjo.2004.058099

Accepted for publication 20 October 2004

Financial support: nil.

Financial interest: nil.

\section{References}

1 Carr RE, Henkind P. Ocular manifestations of ethambutol. Toxic amblyopia after administration of an experimental antituberculous drug. Arch Ophthalmol 1962;67:566-71.

2 Leibold JE. The ocular toxicity of ethambutol and its relation to dose. Ann NY Acad Sci 1966; 135:904-9.
3 Schild H Fox. Raipd-onset reversible ocular toxicity from ethambutol therapy. Am J Med 1991;90:404-6.

4 Van Dijk BW, Spekreijse H. Ethambutol changes the color coding of carp retinal ganglion cells reversibly. Invest Ophthalmol Vis Sci 1983;24:128-33.

5 Kakisu Y, Adachi-Usami E, Mizota A. Pattern electroretinogram and visual evoked cortical potential in ethambutol optic neuropathy. Doc Ophthalmol 1987;67:327-34.

6 Nasemann J, Zrenner E, Riedel KG. Recovery after severe ethambutol intoxication: psychophysical and electrophysiological correlations. Doc Ophthalmol 1989:71:279-92.

7 Heng JE, Vorwerk CK, Lessell E, et al. Ethambutol is toxic to retinal ganglion cells via an excitotoxic pathway. Invest Ophthalmol Vis Sci 1999;40:190-6.

8 Sutter EE, Tran D. The field topography of ERG components in man: I. The photopic luminance response. Vis Res 1992;32:433-46.

9 Hood DC. Assessing retinal function with the multifocal technique. Prog Retin Eye Res 2000; 19:607-46.

10 Kretschmann U, Bock M, Gockeln R, et al. Clinical applications of multifocal electroretinography. Doc Ophthalmol 2000;100:99-113.

\section{Eye involvement mimicking scleritis in a patient with chronic lymphocytic leukaemia}

Acute leukaemia is known to affect the eye in a wide variety of ways and detailed postmortem examination will often reveal subclinical involvement. Ocular involvement by chronic leukaemia is much less common. ${ }^{1}$ We describe the clinical presentation, evaluation, and response to therapy of what is to our knowledge the first case of clinically significant scleral infiltration by chronic lymphocytic leukaemia mimicking scleritis.

\section{Case report}

An 87 year old man presented with a 2 week history of right eye redness, pain, and decreased vision. He complained of horizontal diplopia for 2 years, which had been corrected with prisms, but never investigated. Past medical history was notable only for chronic lymphocytic leukaemia (CLL), which was first diagnosed 17 years before presentation. He was treated intermittently with chlorambucil, but this was stopped as his white cell count had been stable at $30 \times$ $10^{9} / 1$ with minimal cervical and axillary lymphadenopathy. Vision was 6/18 right eye and 6/9 left eye. Intraocular pressure (IOP) was $18 \mathrm{~mm} \mathrm{Hg}$ in each eye. His vision was reduced by bilateral early cataracts and his right eye showed inferior scleral injection (fig 1A), a fibrinous anterior uveitis, and scattered posterior synechiae.

The patient was treated with topical corticosteroids and cycloplegics, but over the 3 weeks following presentation his vision dropped to 6/60 right eye because of choroidal effusions. He had restricted movement of the right eye, proptosis, and shallowing of the anterior chamber with an increase in IOP to $26 \mathrm{~mm} \mathrm{Hg}$. Ultrasound revealed thickening of the inferior sclera. A CT scan of the right orbit revealed a mass in the inferior fornix that was attached to the sclera (fig lB). Excision biopsy revealed patchy infiltration by foci of densely packed small lymphocytic cells, mainly around small blood vessels (fig 2A). Immunostaining showed a predominance of weakly stained CD20 positive B lymphocytes with scattered CD3 positive T cells consistent with CLL. Haematological analysis revealed a total white cell count of $49.1 \times 10^{9} / 1$. He received orbital radiotherapy $27.5 \mathrm{~Gy}$ in 11 fractions using $6 \mathrm{MV} x$ rays, completing the course 5 months after presentation. The inflammation, choroidal effusions, and orbital mass regressed completely but he was left with thinning of the inferior sclera (fig 2B). He was last seen 2 years after presentation with vision of 6/60 right eye but no evidence of active ocular involvement from his CLL. He refused cataract surgery.

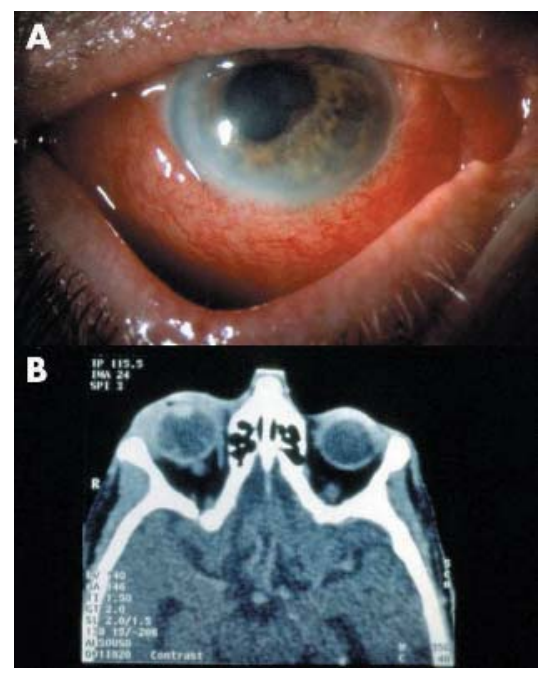

Figure 1 (A) Right eye showing diffuse injection of the inferior sclera. (B) Computed tomography (CT) scan of orbit showing a mass that was confluent with the sclera. 

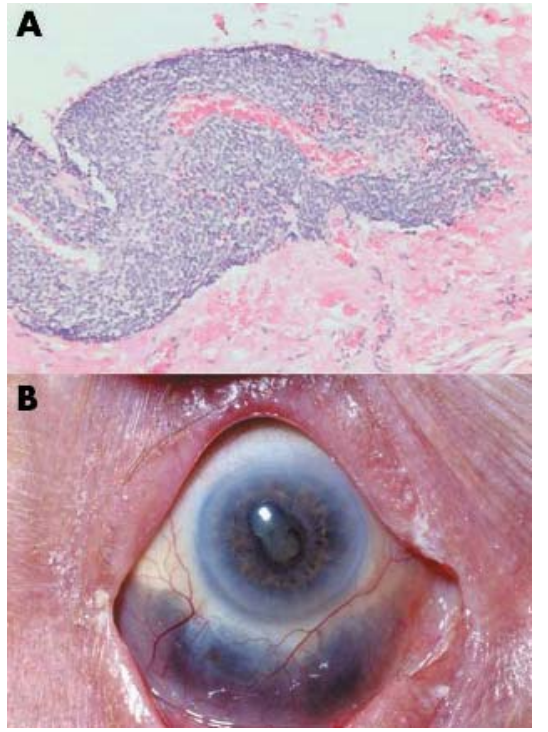

Figure 2 (A) Histopathology of scleral mass showing foci of densely packed small lymphocytic cells, mainly around small blood vessels (haematoxylin and eosin stain, original magnification $\times 200$ ). (B) Right eye following therapy showing thinning of the inferior sclera.

\section{Comment}

This patient presented with what at first appeared to be a scleritis with a secondary fibrinous anterior uveitis. He was 87 years old, which is an unusual age for a first presentation of either scleritis or anterior uveitis. His history of chronic diplopia and past medical history of CLL suggested a possible aetiology for these findings. It is uncommon for CLL to affect the eye in a clinically significant way ${ }^{2}$ although postmortem examination has shown relatively frequent subclinical involvement in between $30 \%$ and $90 \%$ of patients. ${ }^{3}$ Despite this, CLL has been described causing uveitis, glaucoma, iris infiltration, ${ }^{4}$ retinal detachments, conjunctival vascular changes, optic neuropathy, and orbital involvement as well as the more typical retinal haemorrhagic changes.

Episcleral and scleral infiltration is recognised in acute leukaemia and has been seen at necropsy in chronic leukaemia in $14 \%$ of cases in one series but this is rarely symptomatic.

In our patient the response to radiotherapy was encouraging and although his final acuity was not improved this was due in part to the progression of his cataracts. In summary, this is the first case we are aware of in which CLL had infiltrated the sclera of an eye to a sufficient degree that it simulated scleritis. The scleral mass regressed completely following radiotherapy although vision did not recover

B J L Burton Medical Retina Service, Moorfields Eye Hospital, City Road, London ECIV 2PD, UK

E T Cunningham Jr Department of Ophthalmology, New York University School of Medicine, New York, NY, USA

I A Cree Institute of Ophthalmology 11-43 Bath Street, London ECIV 9EL, UK
C E Pavesio

Medical Retina Service, Moorfields Eye Hospital, City Road London ECIV 2PD, UK

Correspondence to: $\mathrm{Mr} \mathrm{C}$ Pavesio, Moorfields Eye Hospital, City Road, London ECIV 2PD, UK; cpavesio@aol.com

doi: $10.1136 /$ bjo.2004.060152

Accepted for publication 16 November 2004

\section{References}

1 Allen RA Straatsma BR. Ocular involvement in leukemia and allied disorders. Arch Ophthalmol 1961;66:490-508

2 Buchan J, McKibbin M, Burton T. The prevalence of ocular disease in chronic lymphocytic leukaemia. Eye 2003;17:27-30.

3 Kincaid MC, Green WR. Ocular and orbital involvement in leukemia. Surv Ophthalmol 1983;27:211-32.

4 Martin B. Infiltration of the iris in chronic lymphatic leukaemia. Br J Ophthalmol 1968;52:781-5.

\section{Regression of choroidal metastases from breast carcinoma using aromatase inhibitors}

Breast cancer has become a leading health concern in the United States, accounting for $30 \%$ of all cancers among women. ${ }^{1}$ Oestrogen is an important hormone involved in the development and growth of breast tumours. ${ }^{2}$ It has been found that $60-70 \%$ of breast cancers have oestrogen receptors. ${ }^{2}$ Following tumour resection, receptor positive patients are commonly treated with hormone therapy such as tamoxifen. Tamoxifen is a competitive antagonist of oestrogen at its receptor site. It is most often used for postmenopausal patients with oestrogen receptor positive breast cancers. However, tamoxifen also has a partial oestrogen agonist effect which could be detrimental, because it can lead to increased risk for uterine cancer, thromboembolism, and treatment failure. ${ }^{3}$

The third generation aromatase inhibitors, including anostrozole, letrozole, and vorozole, have emerged as a new treatment for postmenopausal women with oestrogen receptor positive breast cancer. Their mechanism differs from that of tamoxifen as they minimise peripheral conversion of circulating androgen to oestrogen. ${ }^{3}$ We report one patient with choroidal metastasis from oestrogen receptor positive breast cancer who showed an excellent response to oral aromatase inhibitors.

\section{Case report}

A 66 year old woman presented in January 2003 with a 5 month history of flashing lights in the right eye. Her medical history revealed breast cancer, oestrogen receptor positive, treated with modified radical mastectomy and tamoxifen. In 1989, tamoxifen was stopped per the standard 5 year protocol. In October 2002, she developed metastases to the supraclavicular lymph nodes bone and was started on anastrozole.

On examination, visual acuity was 20/60 RE and 20/20 LE. Fundus examination revealed subretinal fluid overlying a solitary amelanotic choroidal metastasis measuring $12 \mathrm{~mm}$ in base and $3 \mathrm{~mm}$ in thickness (fig 1 ). The patient was continued on anastrozole. In
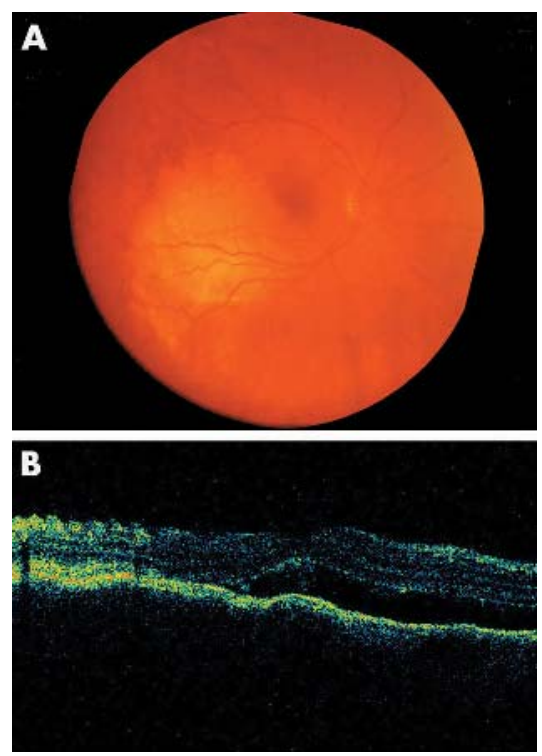

Figure 1 January 2003. (A) Active choroidal metastasis from breast carcinoma at first examination. (B) Optical coherence tomography showing subretinal fluid in the fovea.

September 2003, the choroidal metastasis completely regressed with resolution of subretinal fluid (fig 2). Her visual acuity remained 20/40 RE and 20/20 LE.

\section{Comment}

Uveal metastases are the most common intraocular malignancy. They typically affect the posterior choroids. ${ }^{4}$ The most common primary sites of cancer are from breast $(47 \%)$, lung $(21 \%)$, and gastrointestinal tract $(4 \%) .5$ Classically, choroidal metastases are yellow, plateau shaped, with secondary subretinal fluid. The treatment of choroidal metastases
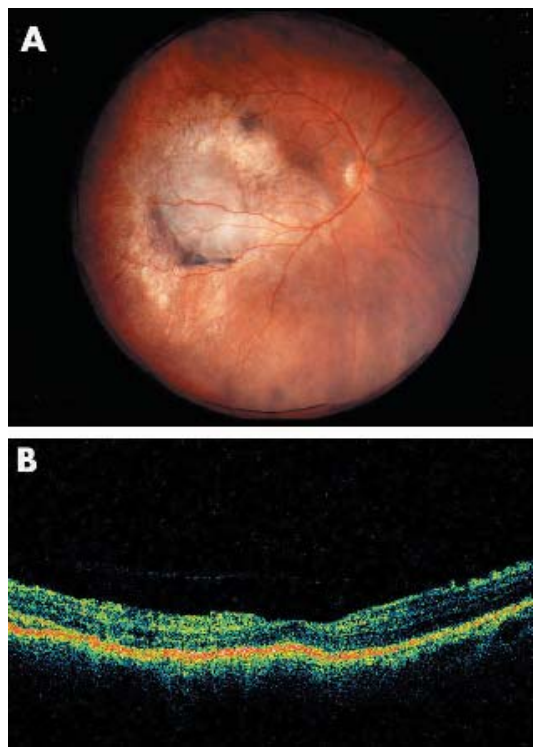

Figure 2 September 2003. (A) Following 9 months of anastrozole treatment, choroidal metastasis has regressed.(B) Optical coherence tomography following 9 months of anastrozole treatment showing resolution of subretinal fluid. 
depends on many factors including location, multiplicity, and activity of each tumour. ${ }^{6}$ Additionally, the projected visual outcome and underlying systemic control is important. In some cases, therapy is limited to the eye, especially if systemic metastases are absent or in remission. In these instances, external beam radiotherapy or plaque radiotherapy are employed. ${ }^{6}$ In most instances, however, the patient has systemically active metastatic disease so therapy is directed towards treatment of both the ocular and systemic disease using chemotherapy or hormone therapy. ${ }^{6}$

The most commonly employed hormonal treatment for breast cancer is tamoxifen. ${ }^{2}$ It is usually employed for postmenopausal patients with breast cancer who display oestrogen receptors. The effect of tamoxifen is due to its anti-oestrogenic activity, by competitive inhibition of oestrogen binding to oestrogen receptors. ${ }^{2}$ Tamoxifen inhibits the expression of oestrogen regulated genes including growth factors and angiogenic factors secreted by the tumour. Tamoxifen can also induce programmed cell death. Tamoxifen additionally has partial agonist effects, which could be beneficial because it prevents bone demineralisation in postmenopausal women. ${ }^{3}$ However, these oestrogenic effects are also associated with increased risks of uterine cancer, thromboembolism, and treatment failure. ${ }^{3}$

Tamoxifen is well tolerated by most patients with breast cancer and only $5 \%$ of patients note related menopausal symptoms, such as hot flashes and vaginal discharge. ${ }^{2}$ Retinopathy has been reported in women with high doses of tamoxifen, but not with conventional doses.

In postmenopausal women the main source of oestrogen is from peripheral conversion of adrenal androgen. New third generation aromatase inhibitors, including anastrozole (Arimidex, Zeneca) and letrozole (Femara, Novartis), act by preventing this conversion, thus lowering circulating oestrogen levels. Studies have shown that they are equal or superior to tamoxifen in clinically efficacy for metastatic breast carcinoma. ${ }^{3}$ They are well tolerated and have been shown to have important benefits over tamoxifen. Comparative trials indicate that anastrozole has similar adverse effects compared to tamoxifen. ${ }^{89}$ Our patient responded dramatically to aromatase inhibitors after failing to respond to tamoxifen. Based on this case and three other cases with a favourable response we anticipate that aromatase inhibitors could be a promising alternative for patients with choroidal metastases from oestrogen receptor positive breast carcinoma.

M E Manquez, C L Shields, E C Karatza, J A Shields

Oncology Service, Wills Eye Hospital, Thomas Jefferson University, Philadelphia, PA, USA

Correspondence to: Dr Maria Manquez, Oncology Service, Wills Eye Hospital, 840 Walnut Street, Philadelphia, PA 19107, USA memanquez@hotmail.com

doi: 10.1136/bjo.2004.061127

Accepted for publication 31 October 2004

Supported in part by the Pan American Ophthalmology Foundation and Retina Research Foundation (Dr ME Manquez), the Eye Tumor Research Foundation, Philadelphia, PA (Dr CL Shields) the Macula Foundation, New York, NY (Dr CL Shields), the Rosenthal Award of the Macula Sociely (Dr CL Shields), the Paul Kayser International Award of Merit in Retina Research, Houston, TX (Dr JA Shields).

\section{References}

1 Feig BW, Berger DH, Fuhrman GM. In: Breast cancer. The MD Anderson surgical oncology handbook. Philadelphia: Lippincott Williams and Wilkins, 2003:14

2 Osborne CK. Tamoxifen in the treatment of breast cancer. N Engl J Med 1998;339:1609-18.

3 Smith IE, Dowsett M. Aromatase inhibitors in breast cancer. N Engl J Med 2003;348:2431-42.

4 Shields JA, Shields CL. In: Metastatic tumor to the uvea and retina. Intraocular fumors. A text and atlas. Philadelphia: WB Saunders,

1992:278-320

5 Shields CL, Shields JA, Gross N, et al. Survey of 520 eyes with uveal metastases. Ophthalmology 1997:104:1265-76.

6 Shields CL. Plaque radiotherapy for the management of uveal metastasis. Curr Opin Ophthalmol 1998;9:31-7.

7 Demirci H, Shields CL, Chao AN, et al. Uveal metastasis from breast cancer in 264 patients. Ophthalmology 2003;136:264-71.

8 ATAC (Arimidex, Tamoxifen Alone or in Combination) Trialists' Group. Anastrozole alone or in combination with tamoxifen alone for adjuvant treatment of postmenopausal women with early breast cancer: first results of the ATAC randomized trial. Lancet 2002;359:2131-9.

9 Mouridsen H, Gershanovich M, Sun Y, et al. Superior efficacy of letrozole versus tamoxifen as first-line therapy for postmenopausal women with advanced breast cancer: results of a phase III study of the International Letrozole Breast Cancer Group. J Clin Oncol 2001;19:2596-606.

\section{"Fingertip" cryoprobe assisted orbital tumour extraction}

Cryoprobes are used to grasp, provide traction, and facilitate orbital tumour removal. ${ }^{1-4}$ Standard ophthalmic cryoprobe tips are typically rounded and offer small surface areas for cryo-adhesion. I describe a new spatulated cryotherapy probe with a large oval and uniform surface area for adhesion
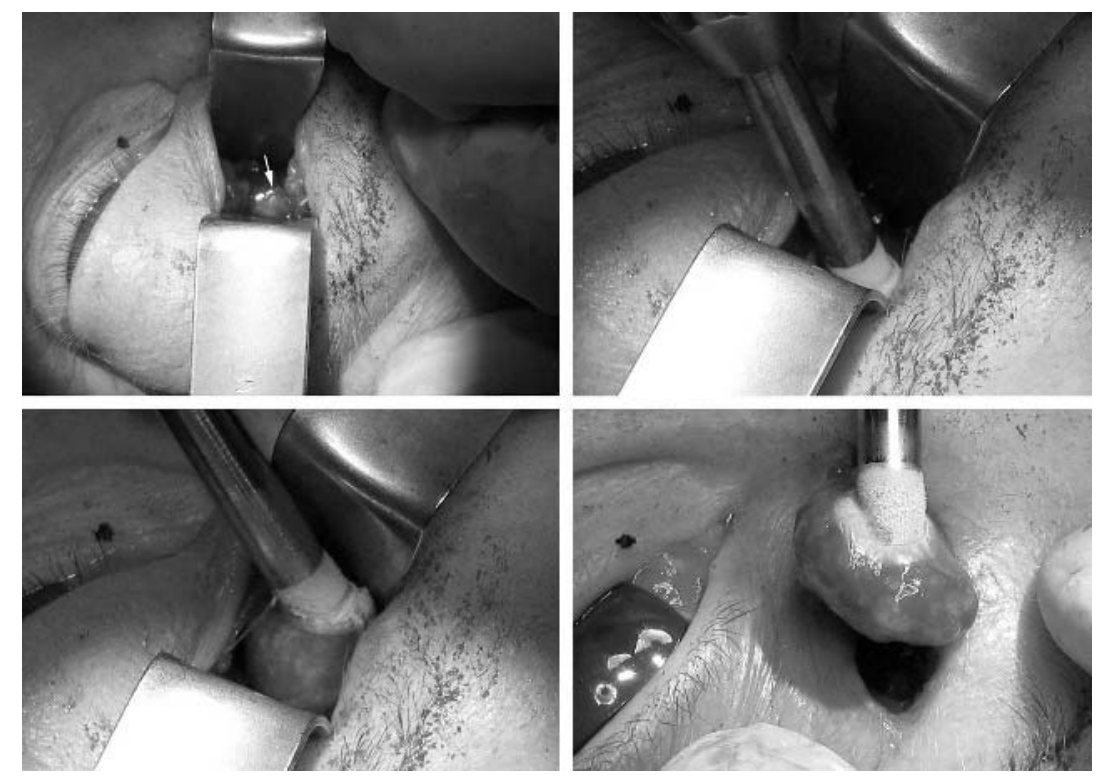

Figure 2 Top left, the tumour (arrow) can be seen deep within the superonasal orbit. Top right, the cryoprobe is introduced such that the spatulated tip is placed flush with the tumour and not touching adjacent orbital tissues. Bottom left, the tumour is delivered from the orbit utilising the cryoadhesion. Bottom right; the tumour is visualised outside the orbit. Note the freeze within the tissue with minimal ice formation on the shaft of the probe. 
portion of the frontal bone and optic nerve. Ultrasonography revealed intrinsic tumour vascularity (low flow). Cavernous haemangioma was suspected and an anterior orbitotomy performed. A combination of sharp and blunt dissection was required to expose the tumour. Then, a medium sized "Fingertip" cryoprobe was used to create an adhesion to the tumour's surface (fig 2). This relatively large cryo-adhesion allowed for easier handling of the tumour and improved traction (fig 2). The tumour remained adherent to the cryoprobe for 60 seconds after the nitrous oxide was discontinued. Freezing induced no change in the appearance of the tumour on gross examination. Histopathology revealed cavernous haemangioma. No clinical side effects (related to cryo-extraction) were noted.

\section{Comment}

Cryo-extraction works best with tumours and cysts that contain fluids (for example, blood, tears), as opposed to solid tumours and those containing lipid or keratin (for example, dermoid). Thus, cavernous haemangiomas are particularly good candidates because freezing occurs on both the tumour's surface (capsule) and within the tumour stroma. With a well formed fibrous capsule, the outer and inner ice balls provide a strong attachment to the probe.

The fingertip cryoprobes differ from previously available applicators in that they offer relatively large, spatulated tips. This allows for more homogeneous cryo-adhesion over a larger surface area. The larger surface area of application also increases tissue penetration, resulting in a stronger adhesion.

This study examines a new type of cryoprobe used to assist orbital surgery during tumour extraction. It was found capable of adhering to the tumour. Held by the surgeon, tumour traction could be induced with one hand, while the other hand wielded instruments used to sever residual tumour attachments. ${ }^{5} \quad$ Fingertip cryoprobes offer an alternative to current devices used for cryoextraction of orbital tumours.

Correspondence to: P T Finger, MD, The New York Eye Cancer Center, 115 East 61 st Street, New York City, NY 10021, USA; pfinger@eyecancer.com

doi: 10.1136/bjo.2004.055426

Accepted for publication 1 October 2004

The work is supported by The EyeCare Foundation, Inc and Research to Prevent. Blindness, New York, USA.

Dr Finger has no proprietary interest in the instrument described in this study.

\section{References}

1 Maroon JC, Onik G, Quigley MR, et al. Cryosurgery re-visited for the removal and destruction of brain, spinal and orbital tumours. Neurol Res 1992;14:294-302.

2 Henderson JW, Neault RW. The use of the cryoprobe in the removal of posterior orbital tumors. Ophthalmic Surg 1976;7:45-7.

3 Loewenstein A, Geyer O, Lazar M. Cavernous haemangioma of the orbit: treatment by transconjunctival cryoextraction. Eye 1993;7(Pt 4):597-8

4 Gdal-On M, Gelfand YA. Surgical outcome of transconjunctival cryosurgical extraction of orbital cavernous hemangioma. Ophthalmic Surg Lasers 1998;29:969-73.

5 Finger PT. "Finger-tip" cryoprobe assisted enucleation. Am J Ophthalmol, (in press).

\section{Congenital third nerve palsy, moyamoya disease and optic nerve head staphyloma}

The association of congenital optic nerve head anomalies, especially of the morning glory disc variety, with moyamoya disease is well recognised and has been described in a number of patients. ${ }^{1-4}$ To the best of our knowledge, the occurrence of a congenital third nerve palsy with moyamoya disease and a congenital optic nerve head anomaly has not been reported. We describe a patient who demonstrated the ipsilateral occurrence of these three congenital abnormalities, strengthening the association of congenital optic nerve and carotid abnormalities and suggesting a common underlying aetiology.

\section{Case report}

A 3 year old boy was examined because of left sided exotropia and blepharoptosis. His birth, developmental, and medical histories were unremarkable. Visual acuity was 20/25 right eye and 20/200 left eye. There was a near total ptosis of the left upper eyelid. There was a large exotropia and a small hypotropia of the left eye that he was unable to adduct, elevate, or infraduct. His right pupil reacted briskly to light, but the left pupil was dilated and sluggishly reactive to light. A left relative afferent pupillary defect was present. Fundus examination disclosed an anomalous left optic disc and peripapillary area, with what appeared to be an enlarged scleral opening and a staphylomatous defect around the optic papilla (fig 1). The rest of the retina was normal. The right fundus was normal.

A magnetic resonance imaging scan and magnetic resonance angiogram of the brain revealed an absence of the left intracranial carotid artery and its bifurcation into middle and anterior cerebral arteries. The lenticulostriate arteries were increased in size, consistent with moyamoya vessels (fig 2).

\section{Comment}

This patient has an optic nerve head malformation that appears to be most compatible with a peripapillary staphyloma or a variant of a morning glory disc anomaly (MGDA). MGDA is one of the cavitary optic disc malformations comprising a congenital, funnel-shaped excavation of the posterior peripapillary sclera that incorporates the optic disc. In addition to the anomalous retinal

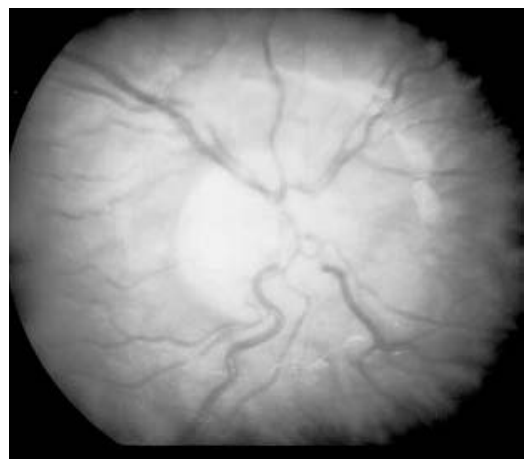

Figure 1 Enlarged optic nerve head scleral opening with radial exit of retinal blood vessels. Note staphylomatous appearance of peripapillary area.

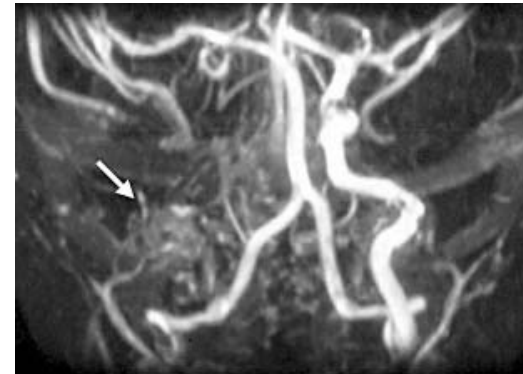

Figure 2 Cerebral angiogram shows total absence of left internal carotid artery (arrow) with increased size of lenticulostriate arteries compatible with moyamoya disease.

vasculature that distinguishes the MGDA from other excavated optic disc anomalies such as optic disc coloboma, the association of morning glory disc with carotid circulation anomalies, especially moyamoya disease has been reported. ${ }^{1-5}$ Moyamoya disease is a rare cerebrovascular disorder characterised by stenosis or occlusion of the distal internal carotid arteries. Progressive brain ischaemia triggers formation of a collateral vascular network in the basal ganglia region referred to as moyamoya (Japanese word for "cloud of smoke") vessels. The most common clinical features are transient ischaemic attacks and stroke in children, and intracranial haemorrhage in adults. Our patient appears to have an extreme form of this condition with total absence of the left internal carotid artery and collateral vessel formation typical of moyamoya disease.

Congenital third nerve palsy was once thought not to be associated with other neurological abnormalities. More recent studies have however revealed a high incidence of associated neurological deficits such as hemiparesis, seizures, hemianopia, and hydrocephalus. ${ }^{5}$ An association of congenital third nerve palsy with MGDA or peripapillary staphyloma has not been reported to the best of our knowledge. The third nerve palsy in the present case may be due to an ischaemic brainstem event or a developmental defect affecting the left third nerve nucleus.

The constellation of abnormalities in our patient confirms the association of moyamoya vessels with optic nerve head malformations such as MGDA or peripapillary staphyloma, and the possible neurological complications of this malformation complex, as evidenced by the third nerve palsy in this case. It is possible that an intracranial vascular dysgenesis may underlie some cases of the morning glory disc anomaly. We strongly recommend magnetic resonance angiography in conjunction with magnetic resonance imaging to identify carotid vascular anomalies in patients with MGDA or peripapillary staphylomas, especially in the presence of other neurological signs such as a congenital third nerve palsy.

K Sabti, B A Haii Al-Bahar Eye Center and the Department of Ophthalmology, University of Kuwait, Kuwait City, Kuwait

J-M Hwang

Department of Ophthalmology, Seoul Municipal Boramae Hospital, College of Medicine Seoul National University, Seoul, Korea 
J-M Hwang, E I Traboulsi

The Center for Genetic Eye Diseases, Cole Eye Institute, Cleveland, $\mathrm{OH}$, USA

J Reid

Department of Pediatric Radiology, The Cleveland Clinic Foundation, Cleveland, $\mathrm{OH}$, USA

Correspondence to: Elias I Traboulsi, MD, Center for Genetic Eye Disease, Cole Eye Institute, The Cleveland Clinic Foundation, i32, 9500 Euclid Avenue, Cleveland, OH 44195, USA; traboue@ccf.org

doi: 10.1136/bjo.2004.059246

Accepted for publication 1 November 2004

\section{References}

1 Hanson MR, Price RL, Rothner $A D$, et al. Developmental anomalies of the optic disc and carotid circulation. A new association. J Clin Neuroophthalmol 1985:5:3-8.

2 Massaro M, Thorarensen O, Liu GT, et al. Morning glory disc anomaly and moyamoya vessels. Arch Ophthalmol 1998;116:253-4.

3 Bakri SJ, Siker D, Masaryk T, et al. Ocular malformations, moyamoya disease, and midline cranial defects: a distinct syndrome. Am J Ophthalmol 1998;116:253-4.

4 Krishnan C, Roy A, Traboulsi E. Morning glory disk anomaly, choroidal coloboma, and congenital constrictive malformations of the internal carotid arteries (moyamoya disease). Ophthalmic Genet 2000;21:21-4

5 Tsaloumas MD, Willshaw HE. Congenital oculomotor palsy: associated neurological and ophthalmological findings. Eye 1997;11(P† 4):500-3

\section{Radial optic neurotomy for the treatment of acute functional impairment associated with optic nerve drusen}

Radial optic neurotomy was recently introduced as a treatment option in patients with central retinal vein occlusion. ${ }^{1}$ As described by Opremcak et al, ${ }^{1}$ central retinal vein occlusion might be related to increased pressure on the central retinal artery and vein as well as on optic nerve fibres in the confined space provided by the scleral ring. It was therefore suggested that a relaxation of the scleral outlet by a radial optic neurotomy might be an effective surgical treatment option. In the light of this information we hypothesised, that radial optic neurotomy may also be applicable in patients with visual field defects and deterioration of visual acuity associated with optic nerve drusen, where compression induced damage to optic nerve fibres is the underlying pathogenetic principle

\section{Case report}

A 27 year old female patient presented with extensive bilateral optic nerve drusen (fig lA). While visual acuity was light perception on the right eye over 4 years, she had experienced an acute and rapid deterioration of visual acuity from $20 / 32$ to $20 / 500$ and a progressive visual field loss within the past 6 weeks before she was seen in our institution (fig 1B). The progressive visual field defect had been documented carefully by the referring ophthalmologist. Besides the optic nerve drusen, there was no other ophthalmic pathology, no history of glaucoma, or any other relevant disease. With respect to the rapid functional deterioration we discussed radial optic neurotomy as a potential treatment option. Surgery was performed, after written informed consent, by one of the authors (AK) and consisted of standard three port pars plana vitrectomy and radial optic neurotomy. The incision was performed at the nasal edge of the optic disc in a radial fashion avoiding major retinal vessels. There were no intraoperative complications except a small haemorrhage at the incision site. The patient was then carefully followed postoperatively at 3 week intervals. Already at the first follow up visit a regression of the visual field defect and slight improvement of visual acuity was noted (fig 2A). At last presentation 10 weeks after surgery, the visual field defect was limited to the inferior nasal quadrant and visual acuity had improved to 20/32 (fig 2B). Funduscopy revealed a scar at the incision site, but no other pathologies (fig 2C).

\section{Comment}

Drusen of the optic nerve are the result of axonal degeneration of retinal ganglion cells and are composed of acellular concentric calcified laminations. ${ }^{3}$ While most cases are idiopathic, drusen have been described in association with several acquired conditions such as hypertensive retinopathy, vascular occlusion, optic atrophy, or chronic papilloedema. ${ }^{4}$ If drusen are located deep in the optic nerve, progressive enlargement may result in pressure induced atrophy of adjacent nerve fibres producing visual field constriction and anterior ischaemic optic neuropathy. ${ }^{2}$ Drusen have also been reported to occur predominantly in eyes with abnormally small optic discs. ${ }^{5}$ It had been previously hypothesised, ${ }^{1}$ that a radial incision at the nasal edge of the optic disc might result in a decompression of the scleral outlet and the associated neurovascular compression in patients with central retinal vein occlusion. Transferring this hypothesis to our patient, radial optic neurotomy seemed a reasonable therapeutic approach to us, as a relaxation or decompression of the scleral outlet might counteract the relevant pathogenetic principles of progressive visual field defect in optic nerve drusen. To our knowledge, radial optic neurotomy has not been used for the treat ment of progressive visual field defects in association with optic nerve drusen before We were very aware of the experimental character of this surgical intervention and carefully informed the patient before the operation. However, with respect to the rapid deterioration of visual acuity and progression of visual field constriction, there seemed to be no other treatment option available and the surgical intervention appeared justified.

The validity of the "scleral outlet compartment syndrome" concept in central retinal vein occlusion, as well as the effect of the radial neurotomy, has been questioned ${ }^{6}$ by Hayreh and is currently under discussion. However, the excellent functional outcome in this case may suggest that radial optic neurotomy potentially provides a relaxation and decompression of the optic nerve, allowing a recovery in conditions associated with pressure induced nerve fibre damage as in the case presented. In retinal vein occlusion, other additional mechanisms of action, such as the formation of chorioretinal shunts as a result of radial optic neurotomy, were described to contribute to visual recovery.

In summary, this case reports indicates that radial optic neurotomy might be considered in patients presenting with sudden visual loss and constriction of visual field in association with optic nerve drusen. We considered the surgical approach in this single case, as there was no other treatment option we could offer the patient that might restore vision.
A

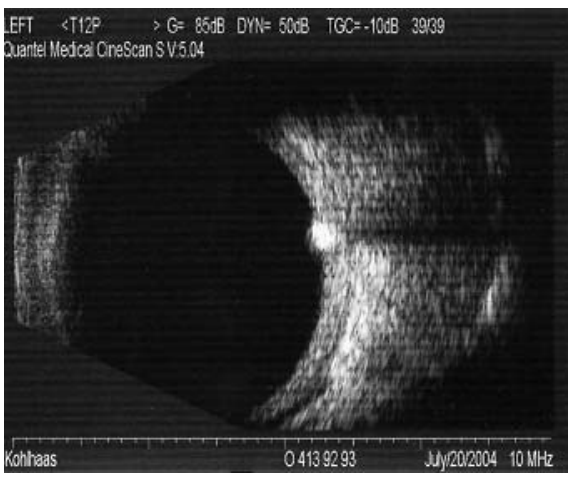

B

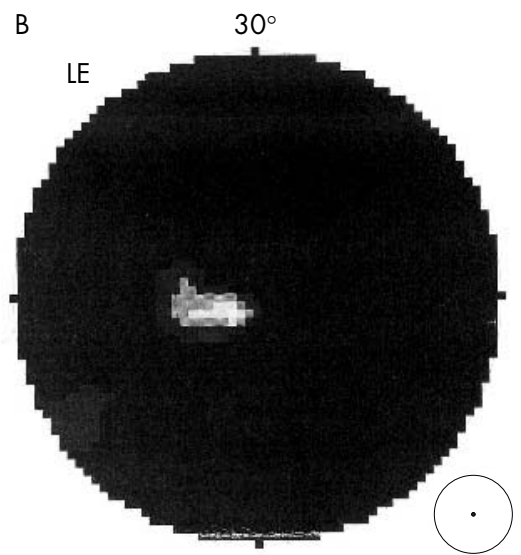

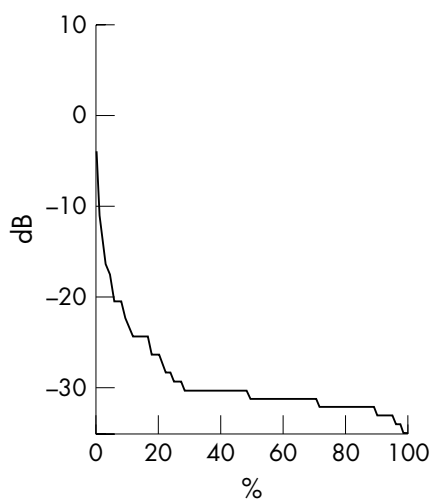

Figure 1 Highly reflective signal seen during B-scan echography at the optic nerve head of the left eye as typical for optic nerve drusen (A). The drusen were associated with a concentric visual field defect (B), visual acuity was 20/500. 


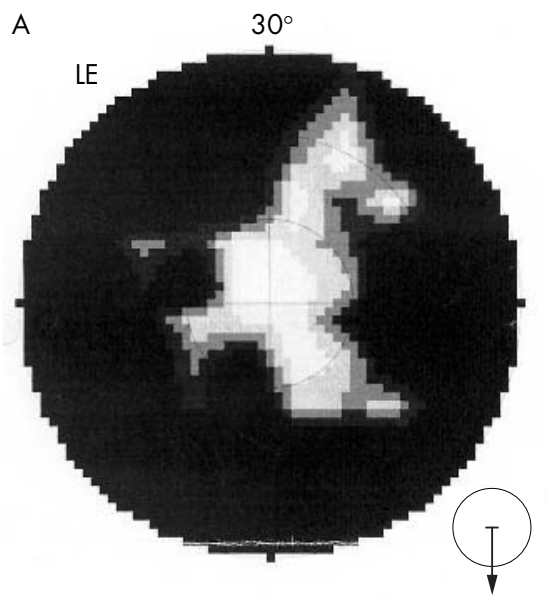

B

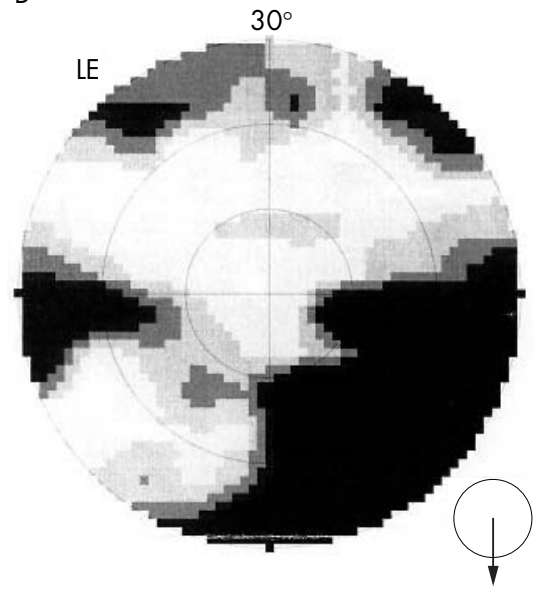

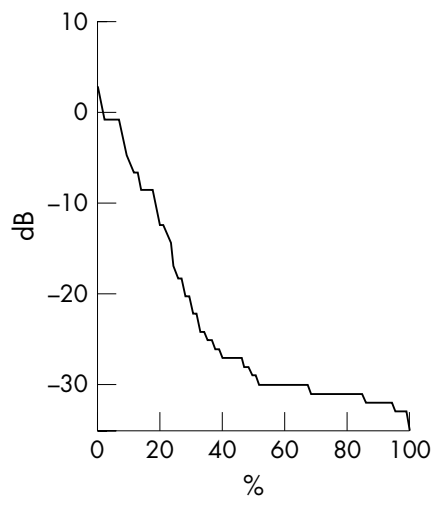

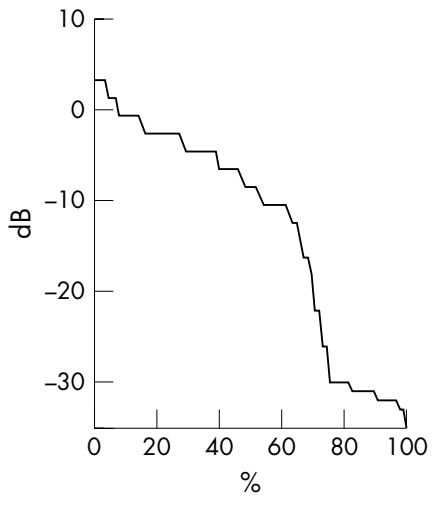

C

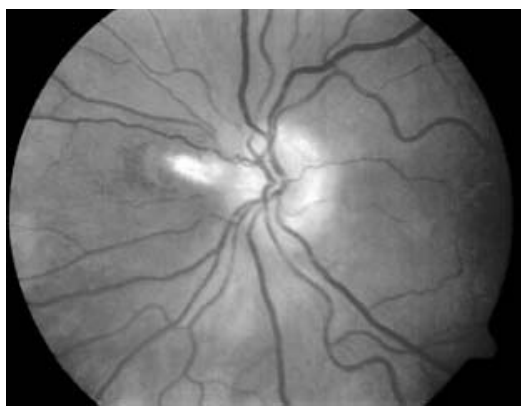

Figure 2 There was a regression of the visual field defect noted 3 weeks after radial optic neurotomy (A). Ten weeks postoperatively the visual field defect was predominantly limited to the lower nasal quadrant (B). Funduscopy revealed a scar at the incision site at the nasal rim of the optic disc (C).

\section{Haritoglou, S G Prieglinger, M Grueterich,} A Kampik Department of Ophthalmology, Ludwig-MaximiliansUniversity, Mathildenstrasse 8, 80336 Munich, Germany

\section{G K Kriegelstein}

Department of Ophthalmology, University of Cologne, Joseph-Stelzmann-Strasse 9, 50931 Cologne, Germany

Correspondence to: Christos Haritoglou, MD, Department of Ophthalmology, Ludwig-Maximilians-University, Mathildenstrasse 8, 80336 Munich, Germany Christos.Haritoglou@med.uni-muenchen.de

doi: $10.1136 /$ bjo. 2004.060335

Accepted for publication 2 November 2004

Financial interest: none.

\section{References}

1 Opremcak EM, Bruce RA, Lomeo MD, et al. Radial optic neurotomy for central retinal vein occlusion. A retrospective pilot study of 11 consecutive cases. Retina 2001 ;21:408-15.

2 Moody TA, Irvine AR, Cahn PH, et al. Sudden visual field constriction associated with optic disc drusen. J Clin Neuroophthalmol 1993;13:8-13.

3 Tso MOM. Pathology and pathogenesis of drusen of the optic nerve head. Ophthalmology 1981;88:1066-80.

4 Apple DJ, Rabb MF, Walsh PM. Congenital anomalies of the optic disc. Surv Ophthalmol 1982;27:3-41.
5 Jonas JB, Gusek G, Guggenmoos-Holzmann I, et al. Optic nerve head drusen associated with abnormally small optic discs. Int Ophthalmol 1987; 11:79-82

6 Hayreh SS. Radial optic neurotomy for central retinal vein occlusion. Retina 2002;22:374-7.

7 Garcia-Arumi J, Boixadera A, MartinezCastillo V, et al. Chorioretinal anastomosis after radial optic neurotomy for central retinal vein occlusion. Arch Ophthalmol 2003;121:1385-91.

\section{Early chorioretinal anastomosis in non-ischaemic CRVO: a randomised trial}

In non-ischaemic central retinal vein occlusion (niCRVO), the two principal determinants of final visual acuity are visual acuity and the presence of macular oedema at initial presentation. ${ }^{12}$ Data from the Central Vein Occlusion Study Group ${ }^{2}$ suggested that of patients with CRVO with an initial acuity better than 6/15, 65\% maintain this, whereas patients with presenting acuities between $6 / 15$ and $6 / 60,81 \%$ remain the same or get worse ( $19 \%$ improve to $6 / 12$ or better, $44 \%$ remain between $6 / 15$, and $6 / 60$ and $37 \%$ are worse than 6/60). Up to $34 \%$ of niCRVO may progress to the ischaemic variant with its attendant complications within 3 years. $^{2}$ However, in some studies $83 \%$ of indeterminate cases progress to ischaemic CRVO. The creation of a chorioretinal venous anastomosis (CRVA) improved visual acuity in some patients, decreased macular oedema, and reduced the incidence of progression to ischaemic CRVO. ${ }^{3-5}$ These important observations provide the basis for the study we carried out.

During the study period 11 patients (table 1) were enrolled according to the tria protocol (see appendix). All anastomoses were patent (on fluorescein angiography and if not repeated, see appendix). Mean patient age and mean pretreatment (T0) visual acuities, retinal thickness (by optical coherence tomography, OCT), and cyst height (OCT) did not differ between the two groups (table 1, fig 1). Factorial analysis of variance (ANOVA, Genstat) found that all three measures (acuity, retinal thickness, and cyst height, fig l) decreases significantly between T0 and 6 months, but only in the case of cyst height was there a significant difference between the laser treated and control groups $(F(1,9)=5.85, \mathrm{p}<0.05)$. The changes in retinal thickness and visual acuity were also greater in the treatment group but did not achieve significance $(F(1,9)=2.51$ and 1.15 , respectively, both $\mathrm{p}>0.1)$. Larger group sizes may have shown a significant effect.

The principal limitation of this trial is the small number of patients, because of the limited numbers of suitable patients within the study period. However, patients were randomised and well matched. The tria showed a trend towards better vision over 6 months in the laser treated group, although this failed to reach statistical significance. 

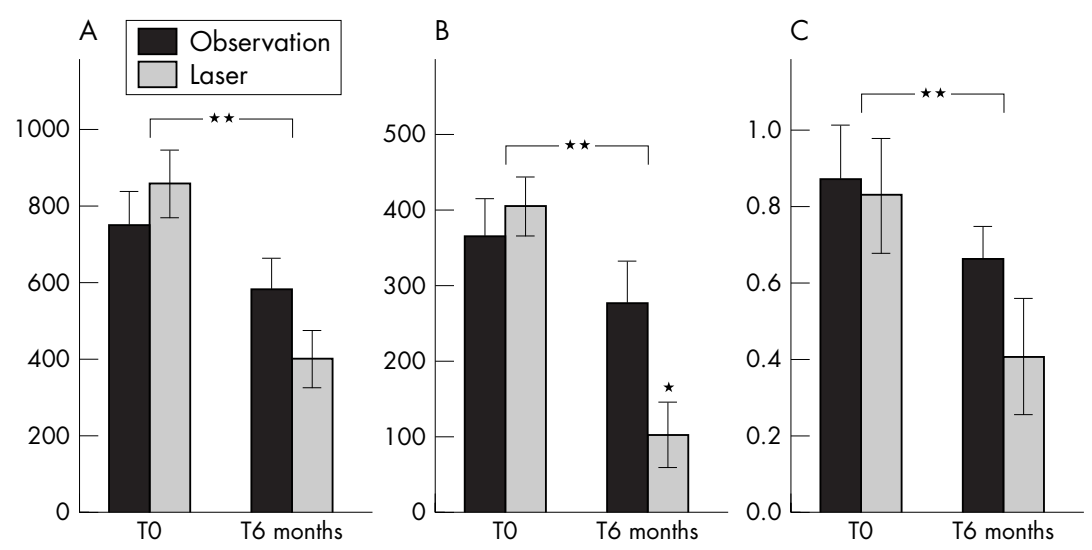

Figure 1 Data of retinal thickness (A), cyst height (B), and visual acuity (C) for patients randomised to laser chorioretinal venous anastomosis or observation. Visual acuity is measured on the ETDRS scale. Retinal thickness and cyst height were measured in pixels (1 pixel $=4 \mu \mathrm{m})$. Error bars show standard errors of means, obtained from factorial analysis of variance. Significant effects are identified as follows: ${ }^{*} \mathrm{p}<0.05 ;{ }^{* *} \mathrm{p}<0.01$.

It remains possible that a larger group of randomised patients would have produced statistically significant numbers. A multicentre trial would be required to achieve this. Another question arises regarding the longer term outcome in these patients. Again this should probably be considered in further studies. Cyst height might predict later visual acuity as there is evidence that it correlates with visual acuity outcome measures. ${ }^{67}$ It is therefore possible that longer follow up may have found a significant improvement in acuity. Retinal thickness predicts and precedes changes in visual acuity in diabetic retinopathy. ${ }^{8}$

If the size and duration of this trial had been increased, it is conceivable that results may have proved more informative. The fact that there was a significant effect on cyst height on OCT is, nevertheless, important and suggests that laser CRVA may reduce macular oedema in CRVO. The finding that improvements in visual acuity are recorded later than reduction in macular oedema ${ }^{8}$ in other studies implies that a significant effect on visual acuity might have been expected in these patients at a later time. We therefore recommend that this be taken into account in future studies.

\section{Acknowledgements}

We gratefully acknowledge the help of Professor Stephen Dunnett (Cardiff University) with statistical analysis of the data.

\section{R J Antcliff, E J Mayer, T H Williamson,} J S Shilling

Department of Ophthalmology, St Thomas's Hospital, Lambeth Palace Road, London El 7EH, UK

Correspondence to: Eric Mayer, University of Bristol, Clinical Sciences, Bristol Eye Hospital, Lower Maudlin Street, Bristol BS1 2LX, UK; e.mayer@bristol.ac.uk

doi: $10.1136 /$ bjo.2004.062539

Accepted for publication 14 January 2005

RJA was supported by the Lady Allerton Fund.

Competing or financial interests: none.

\section{Appendix}

The principal study end point was visual acuity at 6 months and resolution of macular oedema, the secondary end point was the identification of subgroups on optical coherence tomography (OCT 2000, Humphrey Instruments, USA) with a more favourable outcome including reduction of consecutive ischaemia. For entry into the study patients were required to: (1) be within 1 month of symptom onset; (2) have visual acuity of 6/24 or worse; (3) have good retinal perfusion (less than 10 disc diameters of non-perfusion); (4) have no relative afferent pupillary defect or neovascularisation; (5) have macular oedema confirmed by optical coherence tomography and fluorescein

Table 1 Comparison of randomised groups for early laser chorioretinal venous anastomosis versus observation in non-ischaemic CRVO (patient data are illustrated, visual acuities are given as ETDRS measures)

\begin{tabular}{llll}
\hline Parameter & Control & Laser & $t$ test \\
\hline Number of patients & 5 & 6 & \\
Female patients & 2 & 2 & \\
Left eyes & 5 & 3 & \\
Mean age & 76.4 & 68.8 & $1.278, p=0.117, \mathrm{NS}$ \\
Mean pretreatment acuity & 0.872 & 0.827 & $0.216, p=0.417, \mathrm{NS}$ \\
Mean 6 month acuity & 0.664 & 0.41 & $1.388, p=0.099, \mathrm{NS}$ \\
Mean change in acuity over 6 months & 0.208 & 0.417 & $-1.074, p=0.155, \mathrm{NS}$ \\
Mean change in retinal thickness on & 172.8 & 459.3. & $-1.583, p=0.074, \mathrm{NS}$ \\
OCT over 6 months & 87.6 & 302.6. & $-2.418, p=0.019^{*}$ \\
$\begin{array}{l}\text { Mean change in cyst height on } \\
\text { OCT over 6 months }\end{array}$ & & & \\
\hline
\end{tabular}

OCT, optical coherence tomography. fundus angiography (FFA). The following exclusion criteria were applied: diabetes with macular oedema in the fellow eye; inability to give informed consent; pregnancy; sensitivity to fluorescein; cloudy media sufficient to preclude adequate fundal photography; age less than 40 years.

Upon entry into the trial the following were performed: best corrected visual acuity (Snellen and ETDRS); fundus photography, and fluorescein angiography; OCT assessment of the macula. Patients were then randomised (envelope) to laser or observation groups. Laser anastomosis was performed with a $50 \mu \mathrm{m}$ spot size for 0.1 second with 1.5-2.5 W (maximum power possible) with an argon laser. The first shot was fired at the edge of the vein to rupture Bruch's membrane and the second shot at the edge of the vein to rupture the vein. A third shot with a YAG laser was applied $(3-5 \mathrm{~mJ})$ if required. Anastomotic sites were attempted in the following locations in order of preference-inferonasal, inferotemporal, or superonasal. All anastomoses were made at least 3 disc diameters from the optic disc. At 3-4 weeks repeat FFA was performed to determine anastomotic success: where no anastomosis was present this was repeated, until functioning.

Patients were reassessed at 1, 2, 3, 6, and 12 months and acuities and OCT recorded.

It was expected that approximately 40 patients would be required to show a $50 \%$ benefit.

Because of laser surgery it was not possible to mask the patient or observer as to which treatment had been carried out.

No adverse events were reported or noted in this series.

\section{References}

1 The Central Vein Occlusion Study. Baseline and early natural history report. Arch Ophthalmol 1993:111:1087-95.

2 The Central Vein Occlusion Study Group. Natural history and clinical management of central retinal vein occlusion. Arch Ophthalmol 1997:115:486-91.

3 McAllister IL, Constable IJ. Laser-induced chorioretinal venous anastomosis for treatment of nonischemic central retinal vein occlusion. Arch Ophthalmol 1995; 113:456-62.

4 McAllister IL, Douglas JP, Constable IJ, et al. Laser-induced chorioretinal venous anastomosis for nonischemic central retinal vein occlusion: evaluation of the complications and their risk factors. Am J Ophthalmol 1998;126 219-29.

5 McAllister IL, Vijayasekaran S, Yu DY, et al. Chorioretinal venous anastomoses: effect of different laser methods and energy in human eyes without vein occlusion. Graefes Arch Clin Exp Ophthalmol 1998;236: 174-81.

6 Antcliff RJ, Spalton DJ, Stanford MR, et al. Intravitreal triamcinolone for uveitic cystoid macular edema: an optical coherence tomography study. Ophthalmology 2001;108:765-72.

7 Beausencourt $\mathbf{E}$, Remky A, Elsner $A E$, et al. Infrared scanning laser tomography of macular cysts. Ophthalmology 2000; 107:375-85

8 Terasaki H, Kojima T, Niwa H, et al. Changes in focal macular electroretinograms and foveal thickness after vitrectomy for diabetic macular edema. Invest Ophthalmol Vis Sci 2003;44:4465-72 


\section{Informed consent and medical devices: the case of the contact lens}

It is estimated that 1.65 million people in the United Kingdom wear contact lenses, $97 \%$ of whom do so for refractive and cosmetic reasons. ${ }^{1}$ Contact lens wearers are at risk of sight threatening adverse events such as corneal ulceration, which may lead to scarring and visual loss. The risk of corneal ulceration is approximately 1 in 3000, 1 in 2000, and 1 in 500 patients per year for hard, soft daily wear, and soft continuous wear lenses, respectively. ${ }^{2-4}$

Informing individuals of the risks associated with contact lens wear, is important both for the wearer and person prescribing the contact lens. For consent to be legally valid it must be informed. This means that patients need information about the nature of an intervention, possible alternatives and, specifically, its benefits and risks. There is no reason why the principle of informed consent should not apply to the prescribing of a medical device such as a contact lens. We sought to determine the degree to which contact lens wearers have been informed of the risks associated with contact lens wear.

\section{Methods}

Following permission from the respective medical school deans, students at UK medical schools were contacted through electronic mail by their respective university faculties and asked if they wore or had worn contact lenses in the past year, to follow a link to a questionnaire (fig l) sited on the world wide web.

\section{Results}

In all, 560 replies were received (table 1); $21 \%$ of students had received some verbal information regarding the risk of corneal ulceration and $1 \%$ had received this in written form. Only $2 \%$ had been told of all of the four stated risks; $12-14 \%$ of respondents were aware of the risk of corneal ulceration associated with soft daily, continuous, and hard contact lens wear. Of those who knew of this risk $41 \%, 37 \%$, and $58 \%$ underestimated the respective morbidity. Only $23 \%$ of those who think that they had been given sufficient advice knew of the risk of corneal ulceration. Thirteen per cent of students reported the development of one or more complications associated with their contact lens wear. Eight per cent of students stated that had they been aware of the risks stated in the questionnaire they would not have chosen to wear contact lenses. $87 \%$ of students would still have chosen to wear a contact lens after reading the associated risks.

\section{Comment}

Contact lenses are worn by millions of people worldwide. Although the risk of a complication is small, it constitutes a significant public health problem when applied to a large population. Individually it is necessary to balance this risk against the benefits offered by contact lens wear. This can only be achieved with the provision of appropriate information.

Although there are limitations with this type of survey, it would appear that the majority of contact lens wearers, approximately $80 \%$ (98\% for all four of the stated
Q1 For how long have you been wearing contact lenses?

1-5 years

5-10 years

$>10$ years

Q2 Has your contact lens practitioner ever told you of the following risks which are associated with contact lens wear?

Corneal ulcers with loss of vision

Blood vessel formation on the cornea

Corneal scarring with loss of vision

Drooping of the upper eye lid (ptosis)

Yes/No

Yes/No

Yes/No

Yes/No

Q3 Has your contact lens practitioner ever given you written information on the following risks which are associated with contact lens wear? Corneal ulcers with loss of vision Blood vessel formation on the cornea

Yes/No Corneal scarring with loss of vision Drooping of the upper eye lid (ptosis)

Yes/No

Yes/No

Q4 Were you offered a choice of contact lens type by your contact lens practitioner?

Yes/No

Q5 Do you receive your contact lenses from hospital (Yes/No) or high street (Yes/No) contact lens practitioner?

Q6 Do you feel that the information given to you by your contact lens practitioner was sufficient?

Yes/No/Unsure

Q7 On a scale from one to five, with one being very well and five being not at all, how well do you think you understood the information you were given by your contact lens practitioner?

Q8 Have you ever developed any of the following problems? Corneal ulcers

Blood vessel formation on the cornea

Corneal scarring

Drooping of the upper eye lid (ptosis)

1-5

Yes/No

Yes/No

Yes/No

Yes/No

Q9 The estimated risk of corneal ulceration associated with contact lens wear is stated below. Please indicate if you were aware of these given risks. If the answer is yes please select whether you thought the risk was greater than stated, less than stated or the same as stated.

$\begin{array}{llllll} & \text { Risk } & \begin{array}{l}\text { Aware of } \\ \text { risk? }\end{array} & \begin{array}{l}\text { Greater } \\ \text { than }\end{array} & \text { Less than } & \text { Same as } \\ \text { Soft daily wear lenses } & 1 / 2000 & \begin{array}{l}\text { Yes/No } \\ \text { Yes/No }\end{array} & \begin{array}{l}\text { Yes/No } \\ \text { Yes/No }\end{array} \\ \begin{array}{lll}\text { Soft continuous wear lenses } \\ \text { Hard lenses }\end{array} & 1 / 500 & \begin{array}{l}\text { Yes/No } \\ \text { Yes/No }\end{array} & \begin{array}{l}\text { Yes/No } \\ \text { Yes/No }\end{array} \\ \text { Hes/No } & \text { Yes/No } / 3000 & \begin{array}{l}\text { Yes/No } / \text { Yes/No } \\ \text { Yes }\end{array}\end{array}$

Q10 If you were not aware of these risks, would you still have worn contact lenses had you be given the above information?

Yes/No

Figure 1 Questionnaire.

risks) of students surveyed, were unaware of the risks to which they were exposed.

It is important that potential contact lens wearers are fully informed to allow the best choice of refractive correction for them. Consumer comparison between the potential adverse effects of various contact lens types and wear patterns is important in this decision. This is emphasised here as $8 \%$ of those involved in the study would not have chosen to wear contact lenses had they known of the associated risks.

Although there is no legal stipulation regarding the provision of information when prescribing a contact lens, providing information and obtaining informed consent forms part of good clinical practice. Although written consent is not evidence that informed discussion has taken place, written agreements can be used to provide a basis for the process.

We would recommend that informed consent be obtained when prescribing contact lenses and that this should be done with the aid of written information. Concern over the associated risks should not deter people from the benefits of contact lens wear but they are an important consideration and consumers have a right to be informed.
A Roberts, A E Kaye, R A Kaye, K Tu, S B Kaye St Paul's Eye Unit, $8 Z$ Link, Royal Liverpool University Hospital, Prescot Street, Liverpool L7 8XP, UK

Correspondence to: Stephen B Kaye, St Paul's Eye Unit, $8 Z$ Link, Royal Liverpool University Hospital, Prescot Street, Liverpool L7 8XP, UK; Stephen.kaye@ rlbuht.nhs.uk

doi: 10.1136/bjo.2004.062315

Accepted for publication 16 November 2004

\section{References}

1 Schein OD, Glynn RJ, Poggio EC, et al. The relative risk of ulcerative keratitis among users of daily wear and extended wear contact lenses. N Engl J Med 1989:321:773-8.

2 MacRae S, Herman C, Stulting RD. Corneal ulcer and adverse reaction rates in premarket contact lens studies. Am J Ophthalmo $1991 ; 111: 457-65$.

3 Poggio EC, Glynn RJ, Schien OD, et al. The incidence of ulcerative keratitis among users of daily wear and extended wear soft contact lenses. N Engl J Med 1989;321:779-83.

4 Cheng $\mathrm{KH}$, Leung SL, Hoekman HW, et al. Incidence of contact lens associated microbial keratitis and its related morbidity. Lancet 1999;354:181-5. 
Table 1 Survey of 560 medical students who are contact lens wearers. All results are in percentages

\begin{tabular}{|c|c|c|c|}
\hline \multicolumn{4}{|c|}{ Provision of information versus reported complications (\%) } \\
\hline Risk & Verbal & Written & Complications* \\
\hline Ulcer & 18 & 4 & 3 \\
\hline BV & 22 & 4 & 8 \\
\hline Scar & 21 & 4 & 4 \\
\hline Ptosis & 4 & 3 & 1 \\
\hline \multicolumn{4}{|c|}{ Reported awareness of risk of corneal ulceration } \\
\hline SDL & Aware & Unaware & \\
\hline Greater & 18 & 17 & \\
\hline Same & 41 & 18 & \\
\hline Less & 41 & 65 & \\
\hline SCL & Aware & Unaware & \\
\hline Greater & 23 & 21 & \\
\hline Same & 40 & 7 & \\
\hline Less & 37 & 72 & \\
\hline HGP & Aware & Unaware & \\
\hline Greater & 40 & 29 & \\
\hline Same & 2 & 0 & \\
\hline Less & 58 & 71 & \\
\hline \multicolumn{4}{|c|}{ Knowledge of risk of corneal ulcer versus adequacy of advice } \\
\hline & All & Adequate advice & Inadequate advice \\
\hline SDL & 14 & 23 & 21 \\
\hline $\mathrm{SCL}$ & 14 & 24 & 22 \\
\hline & 12 & 23 & 17 \\
\hline \multicolumn{4}{|c|}{ Percentage (\%) who would wear contact lens with knowledge of risks } \\
\hline Yes & 87 & & \\
\hline No & 8 & & \\
\hline
\end{tabular}

*Actual complications reported by students. Blood vessel formation on the cornea (BV). Soft disposable (SDL), soft contact lens (SCL), rigid gas permeable (RGP), or hard contact lens (HGP).

\section{Multiple use of single use solutions: a dangerous practice}

I am writing to raise an issue for routine ophthalmic and optometry practice that contravenes the principles of clinical governance and generates an avoidable infection risk to patients

My concern relates to single use preservative-free ophthalmic solutions, particularly those used for diagnostic purposes in hospital eye clinics and optometry practices. The regulatory guidelines ${ }^{1}$ and the updated guidance note from the Royal Pharmaceutical Society (www.rpsgb.org.uk/pdfs/ophprepguid. pdf) specify that products designed and licensed for single use must be discarded immediately after use.

In practice, however, a recent study ${ }^{2}$ has documented the routine misuse of single use solutions (Minims), generating an unnecessary risk of infection transferral between patients. Single use solutions used multiple times were shown to have a significantly higher rate of contamination than those that were used once then discarded $(45 \%$ versus $4 \%$ ). An associated audit (Qureshi MA, personal communication, 2004) of hospitals in the London region found that $86 \%$ of the ophthalmologists surveyed admitted to multiple uses of single use solutions between different patients.

The study demonstrated the principle that single use products could act as a vehicle for transmission of micro-organisms present on the practitioner's hands or in the lid flora, if shared between patients. We know that gloves are typically not worn in outpatient ophthalmic practice, and that hands are not always washed between each and every patient. We note that even though the eye drop unit itself does not touch the patient's eye or surrounding area, the practitioner often does touch the patient with his/her hands. Furthermore, the potential exists for infection transfer without physical contact. Aside from the risk of infecting the patient, the efficacy of the medication can be compromised in the event of contamination.

My own experience of ophthalmologists and hospital pharmacists indicates that in some cases the interpretation of the term "immediately" has been stretched beyond the simple instruction to "use once and discard." For example, some published papers (Marchese et $a l^{3}$ ) refer to a, now outdated, EMEA instruction to use such products within three hours of opening. The EMEA has since replaced this with an updated guideline $e^{4}$ indicating that the usage time after opening is dependent on the nature of the product, yet this still encourages the user to retain the product for multiple use after first opening.

Clinical governance requires that risks and hazards to patients be reduced to as low a level as possible. Single use products are available for this purpose and should be used as intended and as licensed for one patient only. Repeated use in the same patient over a limited period up to 24 hours after first opening the product may be possible, depending on the product, and at the discretion of the clinician who must ensure that the product is not used on any other patient. I urge your readers to take action as necessary to ensure compliance.

Correspondence to: D V Seal, MD, Applied Vision Research Centre, City University, Northampton Square, London ECIV OHB, UK; davidseal@blueyonder.co.uk

doi: 10.1136/bjo.2004.064535

Accepted for publication 4 January 2005

\section{References}

1 Health Service Circular HSC (IS) 122. London: DHSS, 1975.

2 Wong R, Qureshi MA, Rowe C, et al. Dangers of multiple use of Minims preservative free eye drops in applanation tonometry. 5th International Symposium on Ocular Pharmacology and Therapeutics (ISOPT), 2004.
3 Marchese A, Bozzolasco M, Gualco L, et al. Evaluation of spontaneous contamination of ocular medications. Chemotherapy 2001;47:304-8.

4 EMEA. Maximum shelf life for sterile products for human use after first opening or following reconstitution. CPMP/QWP/159/96corr, 1998 (www.emea.eu.int/pdfs/human/qwp/ 015996en.pdf).

\section{MAILBOX}

\section{Drug induced autoenucleation with resultant chiasmal damage}

I read with interest the report of Tuwir et al ${ }^{1}$ of a case of autoenucleation with chiasmal damage. They note the rarity of this dramatic self mutilating event, and state that there have been only two other reports of contralateral field defect from chiasmal injury in instances of autoenucleation.

There have, however, been a number of other reports of temporal field defect in the remaining eye following either self enucleation or traumatic enucleation by an assailant. The consequences are the same regardless of who removes the eye. One of the two reports Tuwir et al cite, Parmar et al, ${ }^{2}$ was of a young man whose eye was digitally enucleated by an assailant.

In their 1984 review article on autoenucleation, Krauss $e t$ al $^{3}$ reported a 29 year old woman who had enucleated her right globe causing a complete temporal hemianopia in her remaining eye. They also reviewed the medical literature and found 19 cases of bilateral self enucleation (or of self mutilation so severe as to require enucleation) and 31 cases of unilateral self enucleation. Of the 18 eyes where the length of the attached optic nerve was noted, six occurred at or near the chiasm. They also found seven cases of traumatic avulsion of one eye resulting in a temporal hemianopia in the remaining eye.

In 1996, Arkin et al ${ }^{4}$ reported a 25 year old man with a temporal defect in his right eye after his left eye was pulled out by an assailant. A magnetic resonance image confirmed damage to the chiasm. And in 2002, Dilly and Imes $^{5}$ reported a 54 year old schizophrenic man who removed his blind left eye causing a complete temporal hemianopia in his remaining right eye. His MRI showed avulsion of the lateral portion of his chiasm.

It appears that even though acts of traumatic enucleation are rare, associated chiasmal injury is common.

Correspondence to: Richard K Imes, CPMC, USA; rimes56418@aol.com

doi: 10.1136/bjo.2005.068502

Accepted for publication 8 February 2005

\section{References}

1 Tuwir I, Chako E, Brosnahan D, et al. Drug induced autoenucleation with resultant chiasmal damage. Br J Ophthalmol 2005;89:121.

2 Parmar B, Edmunds B, Plant G. Traumatic enucleation with chiasmal damage: magnetic resonance image findings and response to steroids. Br J Ophthalmol 2002;86:1317-18.

3 Krauss H, Yee R, Foos R. Autoenucleation. Surv Ophthalmol 1984;29:179-87.

4 Arkin M, Rubin P, Bilyk J, et al. Anterior chiasmal optic nerve avulsion. AJNR 1996;17:1777-81.

5 Dilly J, Imes R. Autoenucleation of a blind eye. J Neuro-ophthalmol 2001;21:30-1. 


\section{P-glycoprotein expression in retinoblastoma}

We have read with interest the paper by Krishnakumar et al. ${ }^{1}$ We have studied 18 children with retinoblastoma using immunohistochemical detection of P-glycoprotein by the mouse monoclonal antibody HYB-24l in frozen section tumour samples. Like Krishnakumar et al, we found limited expression of P-glycoprotein in retinoblastoma cells since only four of the 18 samples were positive. However, six of 18 samples had Pglycoprotein positivity in tumour associated endothelial cells. Contrary to Krishnakumar et al, we had the opportunity of evaluating children who received previous therapy with drugs that can be affected by P-glycoprotein and no obvious difference in its expression was evident. Only one of the three tumour samples coming from patients who had been exposed to P-glycoprotein mediated chemotherapeutic agents (vincristine and/or etoposide) were positive in retinoblastoma cells. In addition, of the six cases in which Pglycoprotein was detected in tumour associated endothelial cells, only one of them came from a patient who had been exposed to P-glycoprotein mediated chemotherapeutic agents (etoposide). Also, one patient whose tumour and tumour associated endothelium were negative had endothelial cells in the optic nerve that were positive. The patient had not been exposed to P-glycoprotein mediated chemotherapy.

There is little information regarding its expression in normal eye blood vessels, but Pglycoprotein expression has been detected in the normal human retinal pigment epithelium and post-laminar optic nerve, ${ }^{2}$ and it is absent in the permeable microvessels of the choroid and the ciliary process. ${ }^{3}$ P-glycoprotein expression in endothelial cells of newly formed capillaries induced by tumours has also been described. ${ }^{4}$ Its prognostic relevance in retinoblastoma is unknown, and it cannot be determined from our data, since all of these eyes were already enucleated and no patient had an extraocular relapse.

The potential role of P-glycoprotein in tumour associated endothelium creating a blood-tumour barrier is uncertain. Our results suggest that if cyclosporine is indeed improving the efficacy of chemotherapy, it may be acting by improving its delivery through P-glycoprotein expressing tumour associated endothelial cells or through altering pharmacokinetics of chemotherapeutic drugs, rather than exclusively acting to reverse drug resistance at the level of the tumour cell.

Our data do not support the hypothesis that cyclosporine will be a useful adjunct to chemotherapy for the treatment of retinoblastoma via competitive inhibition of $\mathrm{P}$ glycoprotein in tumour cells.

I J Dunkel, D H Abramson, C Cordon-Cardo Memorial Sloan Kettering Cancer Center, Box 185, 1275 York Avenue New York, NY 10021, USA

G L Chantada, M T G de Davila, A C Fandino Hospital JP Garrahan, Combate de los Pozos 1881, C1245AAL, Buenos Aires, Argentina

Correspondence to: Dr Ira J Dunkel, Memorial Sloan Kettering Cancer Center, Box 185, 1275 York Avenue, New York, NY 10021, USA; dunkeli@mskcc.org
Disclosure: There is no financial interest of the authors with any of the drugs and devices mentioned. doi: $10.1136 /$ bjo.2005.068536

Accepted 8 February 2005

\section{References}

1 Krishnakumar S, Mallikarjuna K, Desai N, et al. Multidrug resistant proteins: P-glycoprotein and lung resistance protein expression in retinoblastoma. Br J Ophthalmol 2004;88:1521-6.

2 Kennedy B, Mangini N. P Glycoprotein expression in human retinal pigment epithelium. $\mathrm{Mol}$ Vis 2002;8:422-30.

3 Hofman P, Hoyng P, vander Werf F, et al. Lack of blood-brain barrier properties in microvessels of the prelaminar optic nerve head. Invest Ophthalmol Vis Sci 2001;42:895-901.

4 Camassei FD, Arancia G, Cianfriglia M, et al. Nephroblastoma: multidrug resistance $P$ glycoprotein expression in tumor cells and intratumoral capillary endothelial cells. Am J Clin Pathol 2002;117:484-90.

\section{Who is the author?}

We read with great interest Schwab's answer' to "Who is Ivan Schwab?" "We thank him for his gracious response to our original letter, ${ }^{3}$ which suggested that readers would benefit if the $B J O$ consistently published authors' qualifications. Although Schwab and the editors understood this to be a question of credentials to write about a subject, we maintain that this issue has more to do with giving the reader perspective. An excellent example of the need to understand the authors' perspective comes from Schwab's reply itself.

Schwab made the presumption that we were both ophthalmologists, which is, in fact, not correct. One of us (DP) is a medical graduate and currently a postgraduate research student. Given that the BJO does not publish authors' qualifications, it is clear how this occurred. We propose that it is common for readers to make inferences about the authors, as Schwab did. It is reasonable and logical to consider not only the information, but its source as well. When authors' qualifications are not explicitly stated, as in the $B J O$, readers can easily make erroneous presumptions about the source. Does the BJO expect its readers to make no presumptions about the backgrounds of the authors? This would seem a difficult task for the inquisitive mind. Does it matter that readers may make erroneous presumptions about the authors? We will leave this question to the reader.

It is interesting to note that, on occasion, the $B J O$ does take care to give the reader more background on an author. For example, in an article on informed consent, ${ }^{4}$ the authors are given the designations of "solicitor" and "ophthalmologist," respectively. Similarly, the author of an article on professional regulation, ${ }^{5}$ is given the designation "Chairman of Committee on Professional Performance, General Medical Council, Portex Professor of Anaesthesia, Institute of Child Health, London." This supports our view that it is important for the reader to understand the perspective of the author. Thus, the $B J O^{\prime}$ s approach does not appear consistent.

In answer to Schwab: indeed, we are "reading his essays, asking questions" and will "stay tuned." In fact, we hope that in due course, the $B J O$ will publish his series as a collection. We understand that the need to conserve space limits references. However, rather than for verification, readers who have been stimulated by his writing would benefit from more references-perhaps online-to further their understanding. For example, in August 2003, Schwab referred to Land and Nilsson's book Animal Eyes (Oxford: Oxford University Press, 2002). This is an excellent resource to improve the reader's understanding and appreciation of his essays, particularly those describing invertebrate eyes.

Finally, we note that other BJO readers have not commented on the subject of authors' qualifications. Perhaps, ironically, other readers think that they do not have the credentials to comment.

D Papalkar, I C Francis

Department of Ophthalmology, Prince of Wales Hospital, Randwick, NSW, Australia and the University of NSW, Sydney, NSW, Australia

Correspondence to: Dr lan C Francis, Suite 12, Chatswood Grove, 12-14 Malvern Avenue, Chatswood 2067, Australia; if@student.unsw.edu.au

doi: 10.1136/bjo.2005.069104

Accepted for publication 15 February 2005

\section{References}

1 Schwab IR. In answer to "Who is Ivan Schwab?" Br J Ophthalmol, 2005;89:249.

2 Hoyt CS, Dick A, Bhisitkul B. Who is Ivan Schwab? Br J Ophthalmol 2004;88:1 106.

3 Papalkar D, Francis IC. Authors' qualifications and the BJO. Br J Ophthalmol 2004;88:1227.

4 Teuten B, Taylor D. "Don't worry my good man you won't understand our medical talk": consent to treatment today. Br J Ophthalmol 2001;85:894-6

5 Hatch D. Professionally led regulation in medicine. Br J Ophthalmol 2001;85:513-15.

\section{Vitrectomy with and without scleral buckle for inferior retinal detachment}

In the article presented by Wickham and associates, ${ }^{1}$ the authors compared vitrectomy and gas for treating inferior break retinal detachments with vitrectomy, gas and scleral buckle. The study showed no significant difference in the final outcome between the two groups. While vitrectomy and gas for inferior break retinal detachments appears promising, there are several issues that we would like to raise.

Firstly, the surgery was performed by a registrar, fellow, or consultant. These surgeons may have varying degrees of experience and the inconsistency may affect the rate of successful surgical outcome. Secondly, additional tears were treated with cryotherapy or laser. As shown by Bonnet et al, ${ }^{2}$ the postoperative proliferative vitreoretinopathy (PVR) rate could be as high as $25.8 \%$ in patients treated with cryotherapy compared to $2.2 \%$ in the laser group. It is unclear what the relative distribution of patients who underwent cryotherapy in the two groups was and this may have been a confounding factor in the study. Thirdly, patients underwent an air/gas exchange with either $\mathrm{SF}_{6}$ or $\mathrm{C}_{3} \mathrm{~F}_{8}$. As $\mathrm{C}_{3} \mathrm{~F}_{8}$ had a much longer duration of tamponade than $\mathrm{SF}_{6}$, the use of one agent over another may have led to a difference in the success rate.

The study excluded patients with PVR grade C. However, for those with grade A or $\mathrm{B}$, a scleral buckle was planned before the operation. This could lead to a selection bias where potentially more difficult cases were 
scheduled into the scleral buckle group. This may be a contributing factor for a higher rate of postoperative PVR (20\%) and epiretinal membrane formation in this group, compared to a rate of $5-10 \%$ reported previously. ${ }^{34}$ The underlying vitreoretinal pathology rather than the placement of the scleral buckle may have been a major reason behind the high PVR rate noted in this group.

The authors stated that the main reasons for performing vitrectomy and gas without scleral buckle was to avoid the possible complications of scleral buckle-namely, longer operating time, ${ }^{5}$ exposure, refractive change, diplopia, and anterior segment ischaemia. ${ }^{6-10}$ Perhaps, in the interest of readers, the authors can provide us with the information if any of these complications developed during the study.

The high rate of final reattachment reported in the study is encouraging. We believe that vitrectomy and gas alone is an effective method to treat selected cases of retinal detachments with inferior retinal breaks. A controlled, randomised, prospective study, comparing the outcome in properly matched groups and with meticulous attention to surgical methods ${ }^{11}$ will help address some of the above issues and help elucidate further if the procedure without the use of scleral buckle will benefit patients with inferior break retinal detachment.

We would like to commend the authors for conducting this very nice study on an important topic that may provide a better alternative treatment. We wish that the issues that we raise will help broaden the discussion on the topic.

Y Y Y Kwong, C W Tsang, W W Lai, D S C Lam Hong Kong, People's Republic of China

Correspondence to: Professor Dennis Lam, 3/F, Hong Kong Eye Hospital, 147K Argyle Street, Kowloon, Hong Kong; dennislam_pub@cuhk.edu.hk

doi: 10.1136/bjo.2005.068528

Accepted for publication 8 February 2005

\section{References}

1 Wickham L, Connor M, Aylward GW. Vitrectomy and gas for inferior break retinal detachments: are the results comparable to vitrectomy, gas, and scleral buckle? $\mathrm{Br} J$ Ophthalmol 2004;88:1376-9.

2 Bonnet M, Guenoun S. Surgical risk factors for severe postoperative proliferative vitreoretinopathy in retinal detachment with grade B PVR. Graefes Arch Clin Exp Ophthalmol 1995;233:789-91.

3 Charteris DG, Sethi CS, Lewis GP, et al. Proliferative vitreoretinopathy. $V$ Developments in adjunctive treatment and retinal pathology. Eye 2002; 16:369-74.

4 The Retina Society Terminology Committee. The classification of retinal detachment with proliferative vitreoretinopathy. Ophthalmology 1983;90:121-5.

5 Hakin KN, Lavin MJ, Leaver PK. Primary vitrectomy for rhegmatogenous retinal detachment. Graefes Arch Clin Exp Ophthalmol 1993;231:344-6.

6 Findall RJ, Norton EW, Curtin, et al. Reduction of extrusion and infection following episcleral silicone implants and cryopexy in retinal detachment surgery. Am J Ophthalmol 1971;71:835-7.

7 Hayashi H, Hayashi K, Nakao F, et al. Corneal shape changes after scleral buckling surgery. Ophthalmology 1997;104:831-7.

8 Domniz Y, Cahana M, Avni I. Corneal surface changes after pars plana vitrectomy and scleral buckling surgery. J Cataract Refract Surg 2001;27:868-72.
9 Fison PN, Chignell AH. Diplopia after retinal detachment surgery. Br J Ophthalmol 1987;71:521-5

10 Kwartz J, Charles S, Mc Cormack P, et al. Anterior segment ischaemia following segmental scleral buckling. Br J Ophthalmol 1994;78:409-10.

11 Leaver P. Expanding the role of vitrectomy in retinal reattachment surgery. $\mathrm{Br} J$ Ophthalmol 1993;77:197.

\section{Charles Bonnet syndrome in patients with glaucoma and good acuity}

We read with interest the paper by Tan et al on Charles Bonnet syndrome (CBS) in Asian patients. Their finding of a lower CBS prevalence than European or North American surveys demands further investigation, although this may reflect the stringent criteria of hallucination complexity they used in making the diagnosis (thus excluding the commonest CBS hallucinations of coloured blobs and grid-like "tesselloptic" patterns ${ }^{2}{ }^{3}$ ) and, as pointed out in the accompanying editorial comment, the relatively low prevalence of macular disease in their cohort. However, it is not this aspect of the report we found most intriguing-it was the observation that CBS occurred with good acuity. In fact, three of the four CBS patients described had a degree of impairment which placed them at risk for CBS (best eye acuity 0.3 or worse $^{4}$ ). It is the remaining patient (patient three, a 72 year old man) who is of particular importance as his relative preservation of acuity bilaterally (20/30 RE, 20/40 LE) challenges the view that significant acuity loss is a prerequisite for "ophthalmological" visual hallucinations. This case mirrors four patients we have recently studied with CBS secondary to glaucoma and bilaterally good acuity. We describe the cases below and offer a pathophysiological mechanism for the association.

In one sense, the finding that CBS occurs with preserved acuity is hardly novel. As cited by Tan et al, ${ }^{1}$ several previous reports have found such an association. However, all is not as it seems, the term CBS being used in different ways by different authors. Some use the term to describe visual hallucinations with insight, irrespective of the presence of eye disease, age or clinical context. ${ }^{5}$ Others use the term to describe the association of visual hallucinations with age and intact cognition, without reference to eye disease or hallucination phenomenology. ${ }^{78}$ Under these definitions it is hardly surprising that a patient with "CBS" has preserved acuity, the patients in these studies having a diverse range of conditions from delirium to Parkinson's disease and beyond. In contrast, ophthalmologists and neurologists have used CBS to emphasise eye or visual pathway disease, with the phenomenology of the hallucinations and age being of secondary importance. ${ }^{910}$ Although each definition of CBS has its merits, the ophthalmological definition reminds us best of Bonnet's original description and helps characterise a distinctive subgroup of visually hallucinating patients with predicable prognosis and specific pathophysiology. ${ }^{211}$ However, even CBS as defined ophthalmologically carries with it an inherent ambiguity: is it eye disease itself or the loss of acuity that is the important factor? The consistent finding of acuity loss as a risk factor ${ }^{42}{ }^{13}$ suggests the latter, or at least that the central retina has a key role in the underlying pathophysiological mechanism.

As part of a larger study into the visual phenomenology of CBS, we have recruited four patients with advanced glaucoma (three POAG and one chronic narrow angle) but preserved visual acuity. The age range of the patients was 81-91 years, three men and one woman. Their visual acuities ranged from $6 / 6$ to $6 / 12$ monocularly with all patients having $6 / 9$ or better in their better eye. All had extensive field defects bilaterally and cup to disc ratios of 0.8 or greater in both eyes. Two patients had bilateral trabeculectomies now off treatment, one was on bimatoprost and Trusopt to both eyes and one on timolol $0.25 \%$ to both eyes. Two patients were bilaterally pseudophakic. The patient with chronic narrow angle glaucoma had previous surgical iridectomies. The duration of their hallucinations ranged from 6 months to 6 years. Three patients hallucinated in colour and one in black and white. The most common hallucination was of tessellopsia ${ }^{2}$ experienced by all the patients, with two patients seeing, in addition, formed buildings and two patients, letter-like shapes. There were also single reports of hallucinations of groups of people, animals, branching shapes $\left(\right.$ dendropsia $\left.^{2}\right)$ and one patient described visual allesthesia. ${ }^{14}$ In three of the patients the hallucinations encompassed the entire visual field, in the fourth they were restricted to the visual field defect. None had hallucinations in other sensory modalities and all had insight into the nature of the experiences. The phenomenology of the hallucinations and the relative frequency of the different hallucination categories are consistent with previous descriptions of ophthalmologically defined CBS. ${ }^{23}$ Nonophthalmological causes of visual hallucinations ${ }^{23}$ were excluded. As far as we are aware this is the largest case series of patients with visual hallucinations secondary to eye disease and bilaterally preserved visual acuity yet to be reported.

Current aetiological theories of CBS emphasise the importance of deafferentation $^{15}$ (both "physiological" through ganglion cell loss and "functional," for example, related to blindfolding or cataract), the loss of visual input resulting in a change in cortical excitability. ${ }^{2}$ Although it has been assumed that deafferentation of sufficient severity to precipitate CBS implies a consequent loss of acuity, our cases and that of Tan et $a l^{1}$ suggest otherwise. Patients with advanced glaucoma can have a significant degree of ganglion cell loss and consequent physiological deafferentation without a loss of acuity, placing them at risk for CBS. This contrasts with age related macular disease where the loss of central retinal ganglion cells leads, indirectly, to an association of CBS with acuity loss. We conclude that reduced acuity is not a necessary prerequisite for ophthalmologically defined CBS and that ophthalmologists should be aware that patients with preserved acuity but significant deafferenting ocular disease are at risk of the syndrome.

S A Madill, D H Ffytche

Eye Department, King's College Hospital, Denmark Hill, London SE5 9RS, UK

Correspondence to: Mr Stephen Madill, Eye

Department, King's College Hospital, Denmark Hill, London SE5 9RS, UK; samadill@hotmail.com

doi: 10.1136/bjo.2005.066027 
Accepted for publication 4 January 2005

\section{References}

1 Tan CSH, Lim VSY, Ho DYM, et al. Charles Bonnet syndrome in Asian patients in a tertiary ophthalmic centre. Br J Ophthalmol 2004;88: 1325-9.

2 ffytche DH, Howard RJ. The perceptual consequences of visual loss: positive pathologies of vision. Brain 1999; 122:1247-60.

3 Santhouse AM, Howard RJ, ffytche DH. Visual hallucinatory syndromes and the anatomy of the visual brain. Brain 2000;123:2055-64.

4 Teunisse RJ, Cruysberg JR, Verbeek AL, et al. The Charles Bonnet syndrome: a large prospective study in the Netherlands. Br J Psychiatry 1995; 166:254-7.

5 Damas-Mora J, Skelton-Robinson M, Jenner FA. The Charles Bonnet syndrome in perspective. Psychol Med 1982;12:251-61.

6 Gold K, Rabins PV. Isolated visual hallucinations and the Charles Bonnet syndrome: a review of the literature and presentation of six cases. Compr Psychiatry 1989;30:90-8.

7 Podoll K, Osterheider M, Noth J. Das Charles Bonnet-Syndrom. Fortschr. Neurol Psychiat 1989;57:43-60.

8 De Morsier G. Le syndrome de Charles Bonnet: hallucinations visuelles des vieillards sans deficience mentale. Annales MedicoPsychologiques 1967;125:677-702.

9 Manford M, Andermann F. Complex visual hallucinations. Clinical and neurobiological insights. Brain 1998;121:1819-40.

10 Menon GJ, Rahman I, Menon SJ, et al. Complex visual hallucinations in the visually impaired: the Charles Bonnet syndrome. Surv Ophthalmol 2003;48:58-72.

11 ffytche DH. Visual hallucination and illusion disorders: a clinical guide. Adv Clin Neurosci Rehab 2004;4:16-18.

12 Holroyd S, Rabins PV, Finkelstein D, et al. Visual hallucinations in patients with macular degeneration. Am J Psychiatry 1992;149:1701-6.

13 Scott IU, Schein OD, Feuer WJ, et al. Visual hallucinations in patients with retinal disease. Am J Ophthalmol 2001;131:590-8.

14 Girkin CA, Miller NR. Central disorders of vision in humans. Surv Ophthalmol 2001;45:379-405.

15 Burke W. The neural basis of Charles Bonnet hallucinations: a hypothesis. J Neurol Neurosurg Psychiatry 2002;73:535-41.

\section{Author's reply}

I thank Madill and Ffytche for their interesting comments on our paper ${ }^{1}$ and am grateful for the opportunity to respond to some of the issues raised. The first relates to possible variations in the prevalence rate of Charles Bonnet syndrome (CBS) in different populations, on which there is currently very little substantive data. We agree that additional population based studies are necessary to determine whether the differences found between ethnic groups are consistent. We wish to highlight that our findings ${ }^{1}$ are supported by another recently published study of 1000 Japanese patients by Shiraishi et $\mathrm{al}^{2}$ who reported a prevalence rate of $0.5 \%$ compared to $0.4 \%$ in our study population. Since the methodology and diagnostic criteria used in that study are very similar to ours, the comparable prevalence rates reported in both studies may be a true reflection of a lower prevalence of CBS in Asians compared to Western populations.

We would like to point out that the large variation in prevalence rates of CBS in the various cross sectional studies may be explained by the characteristics of the different populations being screened, especially the degree of visual impairment. When patients were drawn from either a general ophthalmology $^{1-3}$ or general medical ${ }^{4}$ clinic, presumably comprising patients with relatively good visual acuity, the prevalence rates ranged from $0.4-2 \%$. In patients with poorer visual acuity ${ }^{35-8}$ the prevalence rates varied from $11-12.3 \%$. This observation suggests that poor visual acuity may be a factor that increases the risk of developing CBS. Indeed, Teunisse et al showed that the risk of CBS was higher in patients with a logMAR visual acuity of worse than $0.3^{3}$ and Holroyd et al also found an association with bilateral visual acuity of 20/60 or less. ${ }^{9}$

Nevertheless, even if reduced visual acuity is indeed a risk factor for the development of CBS, we agree that a reduction in visual acuity is not essential and that CBS can occur in patients with relatively good visual acuity. Gold et al thought that reduced visual acuity is not a prerequisite for the diagnosis of $\mathrm{CBS}^{10}$ and Holroyd et al suggested that it may be the presence of visual impairment and not the severity of visual loss that predisposes to CBS. ${ }^{4}$ Schultz and Melzack, in an excellent review of the cases reported in the literature, found that the visual acuities of patients diagnosed with CBS ranged from normal to no light perception. ${ }^{11}$ It is becoming apparent that CBS can develop in patients with visual field defects but otherwise good central acuity. The patients described by Madill and Ffytche and our own patient (patient 3) had glaucomatous visual field defects. A recent paper by Freiman et $a l^{12}$ documented the presence of hallucinations manifesting within visual field defects that occurred following neurosurgery. Two interesting points can be noted from Freiman et al's series: firstly, the hallucinations were confined to the area of visual loss; secondly, they began soon after the surgery and resolved within days to 6 months after their onset. ${ }^{4}$ In contrast, in three of four patients reported by Madill and Ffytche as well as our own patient, the hallucinations were not restricted to the visual field defect. The relation of the onset of CBS symptoms to the development of visual field defects as well as the localisation of hallucinations within visual fields are issues that warrant further study as they may provide additional insight into the pathophysiology of CBS.

Correspondence to: Colin S H Tan, The Eye Institute, National Healthcare Group, Singapore; colintan_eye@yahoo.com.sg

doi: $10.1136 /$ bjo.2005.068510

Accepted for publication 8 February 2005

\section{References}

1 Tan CSH, Lim VSY, Ho DYM, et al. Charles Bonnet syndrome in Asian patients in a tertiary ophthalmic centre. Br J Ophthalmol 2004:88:1325-9.

2 Shiraishi Y, Terao T, lbi K, et al. The rarity of Charles Bonnet syndrome. J Psychiatr Res 2004;38:207-13.

3 Teunisse RJ, Cruysberg JR, Verbeek AL, et al. The Charles Bonnet syndrome: a large prospective study in the Netherlands. Br J Psychiatry 1995; 166:254-7.

4 Holroyd S, Rabins PV, Finkelstein D, et al. Visual hallucinations in patients from an ophthalmology clinic and medical clinic population. J Nerv Ment Dis 1994; 182:273-6.

5 Brown GC, Murphy RP. Visual symptoms associated with choroidal neovascularization. Photopsias and the Charles Bonnet syndrome. Arch Ophthalmol 1992;110:1251-6.
6 Olbrich HM, Engelmeier MP, Pauleikhoff D, et al. Visual hallucinations in ophthalmology. Graefes Arch Clin Exp Ophthalmol 1987;225:217-20.

7 Teunisse RJ, Cruysberg JR, Hoefnagels WH, et al. Visual hallucinations in psychologically normal people: Charles Bonnet's syndrome. Lancet 1996;347:794-7.

8 Nesher R, Nesher G, Epstein E, et al. Charles Bonnet syndrome in glaucoma patients with low vision. J Glaucoma 2001;10:396-400.

9 Holroyd S, Rabins PV, Finkelstein D, et al. Visual hallucinations in patients with macular degeneration. Am J Psychiatry 1992;149:1701-6.

10 Gold K, Rabins PV. Isolated visual hallucinations and the Charles Bonnet syndrome: a review of the literature and presentation of six cases. Compr Psychiatry 1989;30:90-8.

11 Schultz G, Melzack R. The Charles Bonnet syndrome: 'phantom visual images'. Perception 1991;20:809-25.

12 Freiman TM, Surges R, Vougioukas VI, et al. Complex visual hallucinations (Charles Bonnet syndrome) in visual field defects following cerebral surgery. Report of four cases. J Neurosurg 2004;101:846-53.

\section{Tight necktie, intraocular pressure, and intracranial pressure}

I would like to congratulate Theelen et al for their recent article on impact factors on intraocular pressure measurements in healthy subjects, ${ }^{1}$ and I would like to add a thought. As Theelen and colleagues point out with reference to the literature, ${ }^{23}$ increased pressure in the jugular vein leads to increased brain pressure, and by an increase in the episcleral venous pressure, to an elevation of intraocular pressure. Correspondingly, in a previous study by Teng and associates, it was discussed that a tight necktie may increase intraocular pressure by an increased jugular vein pressure and could affect the diagnosis and management of glaucoma. ${ }^{4}$ It may be taken into account, however, that the brain pressure and pressure in the cerebrospinal fluid space surrounding the retrobulbar part of the optic nerve are the counter-pressure against the intraocular pressure across the lamina cribrosa. ${ }^{5}$ If the cerebrospinal fluid space pressure is elevated (as a result of increased jugular vein pressure), the intraocular pressure may also be allowed to be elevated so that the trans-lamina cribrosa pressure difference may remain constant. Independently of the question of whether a tight necktie may or may not increase intraocular pressure, one may assume that if the intraocular pressure gets higher because of an increased jugular vein pressure, it may, at least partially, be balanced by an increase in brain pressure, without increasing the risk for glaucoma.

Correspondence to: J B Jonas, Department of Ophthalmology, Theodor-Kutzer-Ufer 1-3 Mannheim, 68167, Germany; jost.jonas@augen.ma.uni-heidelberg.de doi: $10.1136 /$ bjo.2005.068544 Accepted for publication 8 February 2005

\section{References}

1 Theelen T, Meulendijks CFM, Geurts DEM, et al Impact factors on intraocular pressure measurements in healthy subjects. Br J Ophthalmol 2004;88:1510-1.

2 Mavrocordatos P, Bissonnette B, Ravussin P. Effects of neck position and head elevation on intracranial pressure in anaesthetized 
neurosurgical patients - preliminary results. J Neurosurg Anesthesiol 2000;12:10-4.

3 Toole JF. Effects of change of head limb and body position on cephalic circulation. N Engl J Med 1968:279.307-11.

4 Teng C, Gurses-Ozden R, Liebmann JM, et al. Effect of a tight necktie on intraocular pressure. Br J Ophthalmology 2003:87:946-94.

5 Jonas JB, Berenshtein E, Holbach L. Lamina cribrosa thickness and spatial relationships between intraocular space and cerebrospinal fluid space in highly myopic eyes. Invest Ophthalmol Vis Sci 2004:45:2660-5.

\section{Authors' reply}

We thank Dr Jonas for his interest in our work and his important supplements about pathophysiological aspects regarding neck circulation and intraocular pressure. As the optic nerve head is in close contact with both intraocular and subarachnoidal space, one may imagine a disc protecting balance in chronically elevated jugular pressure. ${ }^{1}$ Short term fluctuations of cervical circulation, as present in our recent study, may not facilitate this guarding benefit. ${ }^{2}$ Thus, repeated huge variations of jugular pressure can presumably be a risk factor for glaucomatous optic nerve head damage. Obviously, this is not the case in slit lamp adapted measurement of intraocular pressure. Nevertheless, the patient's neck position during tonometry should be taken into consideration to improve the interpretation of intraocular pressure measurements.

T Theelen, C F M Meulendijks Radboud University Nijmegen Medical Centre, Philips van Leijdenlaan 15 Nijmegen, 6525 EX, Netherlands

Correspondence to: T Theelen, Radboud University Niimegen Medical Centre, Philips van Leijdenlaan 15 Nijmegen, 6525 EX, Netherlands t.theelen@ohk.umcn.nl doi: 10.1136/bjo.2005.069757

Accepted for publication 23 February 2005

\section{References}

1 Jonas JB, Berenshtein E, Holbach L. Lamina cribrosa thickness and spatial relationships between intraocular space and cerebrospinal fluid space in highly myopic eyes. Invest Ophthalmol Vis Sci 2004;45:2660-5.

2 Theelen T, Meulendijks CFM, Geurts DEM, et al. Impact factors on intraocular pressure measurements in healthy subjects. $\mathrm{Br} J$ Ophthalmol 2004;88:1510-11

\section{NOTICES}

Worldwide clinical trials for new technique for early detection of eye disease

A unique new non-invasive technique for high resolution optical imaging of the eye is receiving global acclaim. By combining two high-resolution imaging technologies, the new technique provides doctors with 3-D images of the retina, macula and the optic nerve.

For more information, contact the Media Office on $01227823581 / 823100$ or email MediaOffice@kent.ac.uk News releases can also be found at: http://www.kent.ac.uk/news

\section{Trachoma control}

The latest issue of Community Eye Health (No 52) discusses new developments in the control of trachoma. For further information please contact: Journal of Community Eye Health, International Resource Centre, International Centre for Eye Health, Department of Infectious and Tropical Diseases, London School of Hygiene and Tropical Medicine,
Keppel Street, London WCIE 7HT, UK (tel: +44 (0)207612 7964; email: Anita.Shah@ Ishtm.ac.uk; online edition: www.jceh.co. uk). Annual subscription (4 issues) UK $£ 28$ / US\$45. Free to developing country applicants.

\section{EVER 2005 meeting}

This will take place on 5-8 October 2005 in Vilamoura, Portugal. For further details please contact: Christy Lacroix, EVER Secretary, Kapucijnenover 33, B-3000 Leuven, Belgium (tel: +32 (0) 16233 849; fax +32 (0)16 234 097; email:ever@ skynet.be).

\section{World Ophthalmology Congress 2006 - Brazil}

The World Ophthalmology Congress (which is replacing the International Congress of Ophthalmology) is meeting in February 2006 in San Paulo, Brazil.

For further information on the congress and committees, scientific program and coordinators of different areas are available at the congress website www.ophthalmology2006. com.br

\section{CORRECTION}

doi: 10.1136/bjo.2005.48777corr l

In the paper titled Prevalence and causes of blindness and low vision in leprosy villages of north eastern Nigeria ( $\mathrm{Br} J$ Ophthalmol 2005;89:417-9) the author has notified us of an error. The second-last sentence in the second column on page 417 should read, 'Definitions of blindness and visual impairment were: VA $\geqslant 6 / 18$, normal; $6 / 60 \leqslant \mathrm{VA}<$ $6 / 18$, visual impairment; $3 / 60 \leqslant \mathrm{VA}<6 / 60$, severe visual impairment...'. 


\section{PostScript}

\section{LETTERS}

If you have a burning desire to respond to a paper published in $B J O$, why not make use of our "rapid response" option?

Log onto our website (www.bjophthalmol. com), find the paper that interests you, and send your response via email by clicking on the "eletters" option in the box at the top right hand corner.

Providing it isn't libellous or obscene, it will be posted within seven days. You can retrieve it by clicking on "read eletters" on our homepage.

The editors will decide as before whether to also publish it in a futher paper issue.

\section{Subconjunctival corticosteroids for benign lymphoid hyperplasia}

Appropriate treatment of subconjunctival benign lymphoid hyperplasia (BLH) has been unclear. Most have noted poor response to oral or topical corticosteroids. Many recommend observation. Radiotherapy has been used, but there are risks of vision loss.

In this case, we found dramatic response to local subconjunctival injection of long acting corticosteroids, which may represent a therapeutic option for subconjunctival benign lymphoid hyperplasia.

\section{Case report}

A 72 year old woman noticed an enlarging mass on the nasal conjunctiva over the
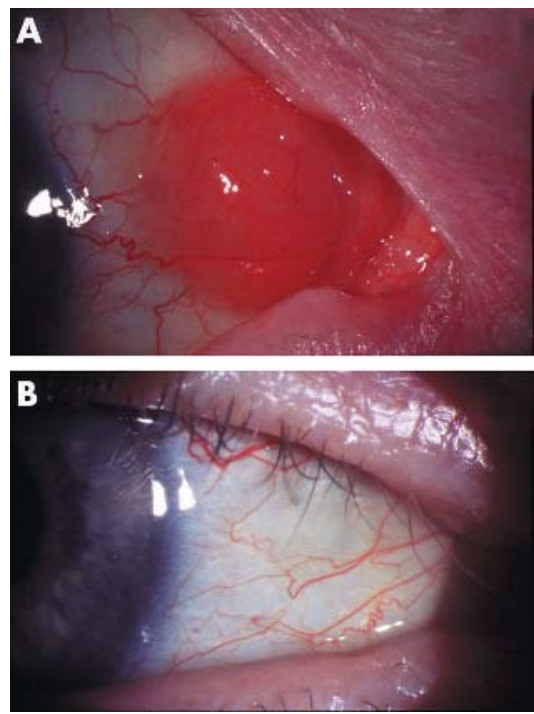

Figure 1 (A) Slit lamp photograph of the patient's right eye on initial presentation. Note the size, salmon colour and raised appearance of the lesion. (B) Slit lamp photograph of the same lesion 2 months later following subconjunctival injection of triamcinolone. The subconjunctival lesion appeared to have completely resolved with no obvious remnants seen on the sclera or conjunctiva. (Reproduced with permission.)

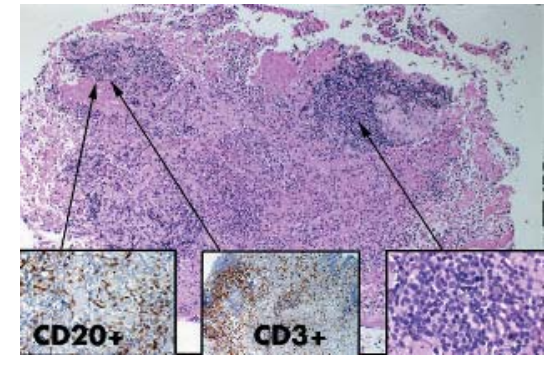

Figure 2 Haematoxylin and eosin staining, $10 \times$ magnification of the lesion biopsied in figure 1A. Note the abundance of lymphocytes seen more clearly in the magnified section in the lower right part of the figure. Also note the predominance of $\mathrm{T}$ cells (CD $3+$ ) with a less numerous population of $B$ cells (CD20+) typical of benign lymphoid hyperplasia.

previous year. She denied any discomfort, diplopia, or visual changes. She was pseudophakic in both eyes, and did not have any past ocular trauma, infection, or eye disease. She had a history of hypothyroidism, multiple myeloma, tuberculosis, and pulmonary sarcoidosis. Two months before presentation she was found to have colon carcinoma treated with colectomy and chemotherapy. She was reportedly free of any tumour metastasis.

Her visual acuity was 20/30 in the right eye and 20/40 in the left, and extraocular motility was full in both eyes. Examination revealed a salmon coloured, raised, and moderately firm patch on the nasal conjunctiva of the right eye without significant neovascularisation (fig 1A). Dilated fundus examination was unremarkable. A head MRI scan showed moderate enlargement of the medial rectus muscle without involvement of the muscle tendon. A simple biopsy $(2 \mathrm{~mm} \times 2 \mathrm{~mm} \times$ $1 \mathrm{~mm}$ ) of the lesion was performed.

Pathological examination revealed benign lymphohistiocytic infiltrates (fig 2). The lymphoid reaction showed a predominance of $\mathrm{T}$ cells $(\mathrm{CD} 3+)$, numerous histiocytes, and a smaller number of B cells (CD20+). There was no evidence of neoplastic plasma cells, metastatic carcinoma, or well defined sarcoid granulomas.

The patient elected to have surgical treatment over other options including observation. The patient received a $20 \mathrm{mg} / 0.5 \mathrm{ml}$ subconjunctival injection of triamcinolone (in $0.5 \mathrm{ml}$ in the nasal bulbar conjunctiva) just superior to the mass. On follow up examination 2 months later, the patient continued to deny any discomfort or visual changes and was very pleased about her response to the treatment. Remarkably, the lesion completely resolved being no longer visible or palpable (fig 1B). This patient unfortunately died 9 months later from complications secondary to a fall, but during this time there was no recurrence of the conjunctival lesion.

\section{Comment}

A patient presenting with a slow growing salmon coloured subconjunctival mass should always raise suspicion of neoplastic causes. Patients with ocular BLH and lymphoma will often have the similar presenting symptoms and demographic profiles. In addition they appear very similar radiologically, ${ }^{1}$ and thus definitive diagnosis requires tissue biopsy. A pathological diagnosis of BLH traditionally requires reactive follicles, polyclonality, and the absence of cytological atypia. $^{2}$ Lymphoproliferative lesions can occur throughout the ocular adnexa, and some studies suggest a more benign course for conjunctival BLH compared to those in the orbit. ${ }^{3}$ Coupland et al found that of 112 cases, $32(29 \%)$ were in the conjunctiva, 52 $(46 \%)$ in the orbit and the remainder in the eyelid, lacrimal gland, and caruncle. ${ }^{4}$ The optimal treatment for BLH is uncertain. Many recommend frequent observation. Others have tried focal radiotherapy with some success, ${ }^{5}$ but there is a significant risk of vision loss. ${ }^{6}$ In a recent review of 117 cases of conjunctival lymphoproliferative lesions, $17 \%$ were BLH, 22\% were atypical lymphoid hyperplasia, and 56\% were lymphoma. ${ }^{7}$ In these cases 9\% were observed, $42 \%$ had complete excisional biopsy, $4 \%$ had biopsy and cryotherapy, $44 \%$ had biopsy and external beam irradiation, and 6\% had biopsy and chemotherapy. $^{7}$

In this case report, we found a dramatic response to local subconjunctival injection of a long acting corticosteroid. The corticosteroid near the reactive follicle must have been sufficient to suppress lymphocyte proliferation. This response may represent a therapeutic option for BLH.

D G Telander, T Z Lee, S E Pambuccian, A J W Huang

Department of Ophthalmology, University of Minnesota, Minneapolis, MN, USA

S E Pambuccian

Department of Pathology, University of Minnesota, Minneapolis, MN, USA

D G Telander

UCLA/Jules Stein Eye Institute, Los Angeles, CA, USA

T Z Lee

Apple Hill Eye Center, 25 Monument Road, Suite 297, York, PA 17403, USÁ

Correspondence to: Andrew J W Huang, MD, Department of Ophthalmology, University of Minnesota, 516 Delaware Street SE, 9th Floor, Minneapolis, MN 55455, USA; huang088@umn.edu'

doi: 10.1136/bjo.2004.051342

Accepted for publication 1 October 2004

\section{References}

1 Knowles DM, Jakobeic FA. Malignant lymphoma and lymphoid hyperplasia occurring in the ocular adnexa. In: Knowles DA, ed. Neoplastic hematology. Baltimore: Williams \& Wilkins, 1992:1009-46.

2 Cockerham GC, Jakobiek FA. Lymphoproliferative disorders of the ocular adnexa. Int Ophthalmol Clin 1997;37:39-59.

3 Knowles DM, Jakobiek FA, McNally L, et al. Lymphoid lyperplasia and malignant lymphoma occurring in the ocular adnexa (orbit, conjunctiva, and eyelids): a prospective multiparametric analysis of 108 cases during 1977 to 1987 . Hum Pathol 1990;21:959-73. 
4 Coupland SE, Krause L, Delecluse H-J, et al. Lymphoproliferative lesions of the ocular adenexa. Analysis of 112 cases. Ophthalmology 1998:105:1430-41.

5 Keleti D, Flickinger JC, Hobson SR, et al. Radiotherapy of lymphoproliferative diseases of the orbit. Surveillance of 65 cases. Am J Clin Oncol 1992;15:422-7

6 Bessel EM, Henk JM, Whitelocke RAF, et al. Ocular morbidity after radiotherapy of orbital and conjunctival lymphoma. Eye 1987;1:90-96.

7 Shields CL, Shields JA, Carvalho C, et al. Conjunctival lymphoid tumors. clinical analysis of 117 cases and relationship to systemic lymphoma. Ophthalmology 2001;108:979-84.

\section{Congenital upper eyelid eversion complicated by corneal perforation}

Congenital upper eyelid eversion is a rare condition more frequently seen in black infants and in Down's syndrome. If recognised early, the condition can be managed conservatively without recourse to surgery. We highlight a case that presented late with severe sight threatening complications. Surgical intervention was consequently the only appropriate way to manage the patient.

\section{Case report}

A 7 month old black female was referred to the emergency room of the King Khaled Eye Specialist Hospital in Riyadh, Saudi Arabia. She had a history of a white spot in the right cornea, and a right upper lid that had flipped up since birth.

She was born by breech delivery and was subsequently diagnosed as having Down's syndrome. On examination she had complete eversion of the right upper lid with mild conjunctival chemosis. The lid could be repositioned with ease but when the child cried it reverted. There was a corneal opacity with a central descematocele and iris adhesion to the endothelium. The child was admitted and started on intensive topical antibiotics. In spite of manual inversion of the upper lid as well as a moist chamber, the lid continued to re-evert. A lateral tarsorrhaphy and penetrating keratoplasty were performed under general anesthesia but postoperatively, the eyelid continued to evert (fig l). Because of the risk of exposure to the transplanted cornea, a full thickness skin graft to the right upper lid was performed (fig 2). Subsequently, the lid retained its normal position and the child could fix and follow adequately with the eye. The transplanted cornea was clear at the 10 week postoperative visit after which the patient was lost to follow up.

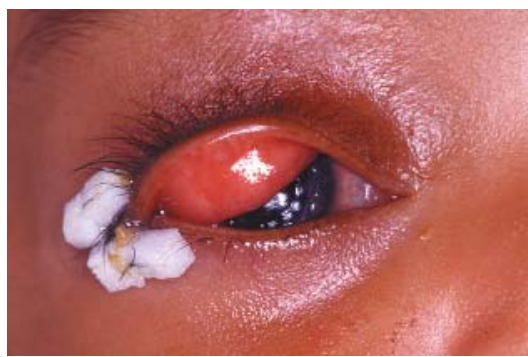

Figure 1 After the tectonic penetrating keratoplasty was performed, the lateral temporal tarsorrhaphy failed to retain the lid in an inverted position.

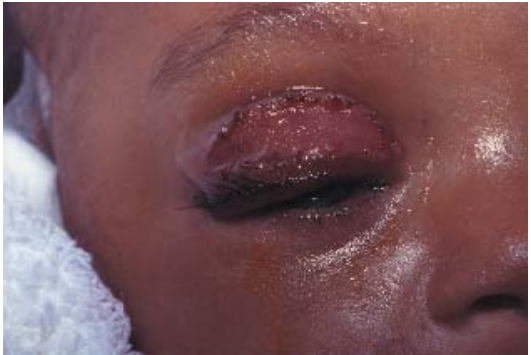

Figure 2 Full thickness skin graft to the upper lid successfully expanded the anterior lamella to permanently solve the problem.

\section{Comment}

Congenital eversion of the upper eyelid was first described in 1896 by Adams ${ }^{1}$ who called the condition "double congenital ectropion." The exact incidence of this condition is not known. Sellar reviewed the literature in 1992 and found 51 reported cases. Since then only two more case reports could be found in the literature. ${ }^{34}$ The eversion is usually present at birth, but late onset of total eversion of the upper eyelids has been described in infancy, ${ }^{5}$ and as late as 11 years of age.

The condition typically is bilateral and asymmetrical but unilateral cases have been described. The underlying pathophysiology is obscure and several possible mechanisms have been proposed and associations recognised. The incidence appears to be higher in black infants, ${ }^{6}$ infants with trisomy $21,{ }^{2}$ and in infants born with collodion skin disease. Abnormalities such as orbicularis hypotonia, ${ }^{8}$ birth trauma, vertical shortening of the anterior lamellar or vertical elongation of the posterior lamellar of the eyelid and failure of the orbital septum to fuse with the levator aponeurosis (with adipose tissue interposition), ${ }^{9}$ absence of effective lateral canthal ligament, and lateral elongation of the eyelid have all been implicated as possible pathophysiological factors. Once everted, orbicularis spasm $^{10}$ may act as a sphincter that leads to a vicious cycle of conjunctival strangulation and oedema secondary to venous stasis. This chemotic conjunctiva usually protects the cornea from exposure and, hence, corneal complications have thus far not been reported in infants. To our knowledge this is the first reported case of congenital eyelid eversion complicated by corneal exposure, descematocele, and perforation.

Congenital eyelid eversion either resolves spontaneously with conservative treatment or with surgical intervention such as a subconjunctival injection of hyaluronic acid, tarsorrhaphy with excision of redundant conjunctiva, fornix sutures, and full thickness skingraft to the upperlid. In this patient because of failure of all conservative measures, the chronicity ( 7 months) and severity (corneal ulcer/perforation), we elected to do anterior lamellar lengthening by means of a full thickness upper lid skin graft. Corneal perforation necessitating penetrating keratoplasty is a rare complication of congenital eyelid eversion, but in this case it was essential to maintain the integrity of the globe and to, hopefully, retain vision and prevent deep amblyopia.

The condition in this reported case of congenital eyelid eversion complicated by a corneal ulcer, large descematocele and perforation, is rare. Congenital upper lid eversion may present "once in a lifetime" to the ophthalmologist or newborn nursery paediatrician. It is nevertheless important to create awareness among healthcare professionals in obstetric and neonatal care of the existence of this potentially sight threatening congenital anomaly, as the condition is very amenable to early treatment.

H Al-Hussain, A A Al-Rajhi, S Al-Qahtani, D Meyer

University of Stellenbosch/King Khaled Eye Specialist Hospital, Faculty of Health Sciences, PO Box 19059, Tygerberg, South Africa

Correspondence to: Professor David Meyer, University of Stellenbosch, Faculty of Health Sciences, PO Box 19059, Tygerberg, South Africa; dm2@sun.ac.za doi: 10.1136/bjo. 2004.053348

Accepted for publication 20 October 2004

\section{References}

1 Adams AL. A case of double congenital ectropion. Med Fortnightly 1896;9:137-8.

2 Sellar PW, Bryars JH, Archer DB. Late presentation of congenital ectropion of the eyelids in a child with Down's syndrome: a case report and review of the literature. J Pediatr Ophthalmol Strabismus 1992;29:64-7.

3 Watts MT, Dapling RB. Congenital eversion of the upper eyelid: a case report. Ophthal Plast Reconstr Surg 1995;11:293-5.

4 Dawodu OA. Total eversion of the upper eyelids in a newborn. Niger Postgrad Med J $2001 ; 8: 145-7$

5 Siverstone B, Hirsch I, Sternberg MD, et al. Late onset of total eversion of upper eyelids. Ann Ophthalmol 1982;14:477-8.

6 Lu LW, Bansal RK, Katzman B. Primary congenital eversion of the upper lids. J Pediatr Ophthalmol Strabismus 1979:16:149-51.

7 Shapiro RD, Soentgen ML. Collodion skin disease and everted eyelids. Postgrad Med 1969;45:216-19.

8 Loeffler M, Hornblass A. Surgical management of congenital upper-eyelid eversion. Ophthalmic Surg 1990;21:736.

9 Blechman B, Isenberg S. An anatomical etiology of congenital eyelid eversion. Ophthalmic Surg 1984;15:111-13.

10 Raab EL, Saphir RL. Congenital eyelid eversion with orbicularis spasm. J Pediatr Ophthal Strabismus 1985;22:125-8.

\section{Homozygous mutation (L527R) of $T G F B I$ in an individual with lattice corneal dystrophy}

Lattice corneal dystrophy (LCD), an inherited form of amyloidosis, is characterised by the development of lattice lines and opacity in the cornea. LCD is classified clinically into four subtypes: I, II, III, and IIIA. Several distinct mutations of TGFBI have been associated with LCDIIIA: P501T, ${ }^{1}$ L527R, ${ }^{2}$ N544S, ${ }^{3}$ A546T, ${ }^{4}$ N622K (T1913G and T1913A), and V627S. ${ }^{5}$ All cases of LCD characterised at the molecular genetic level to date have been attributed to heterozygous point mutations of TGFBI. We now present the first example of a homozygous point mutation of TGFBI in an individual with LCD, a diagnosis supported by clinical, histological, and molecular genetic findings.

\section{Case report}

A 52 year old Japanese man visited our corneal clinic in July 1997 with a main complaint of gradual impairment of vision. His parents, who were related, were no longer alive and he had no children. He had two brothers and four sisters. His reporting 


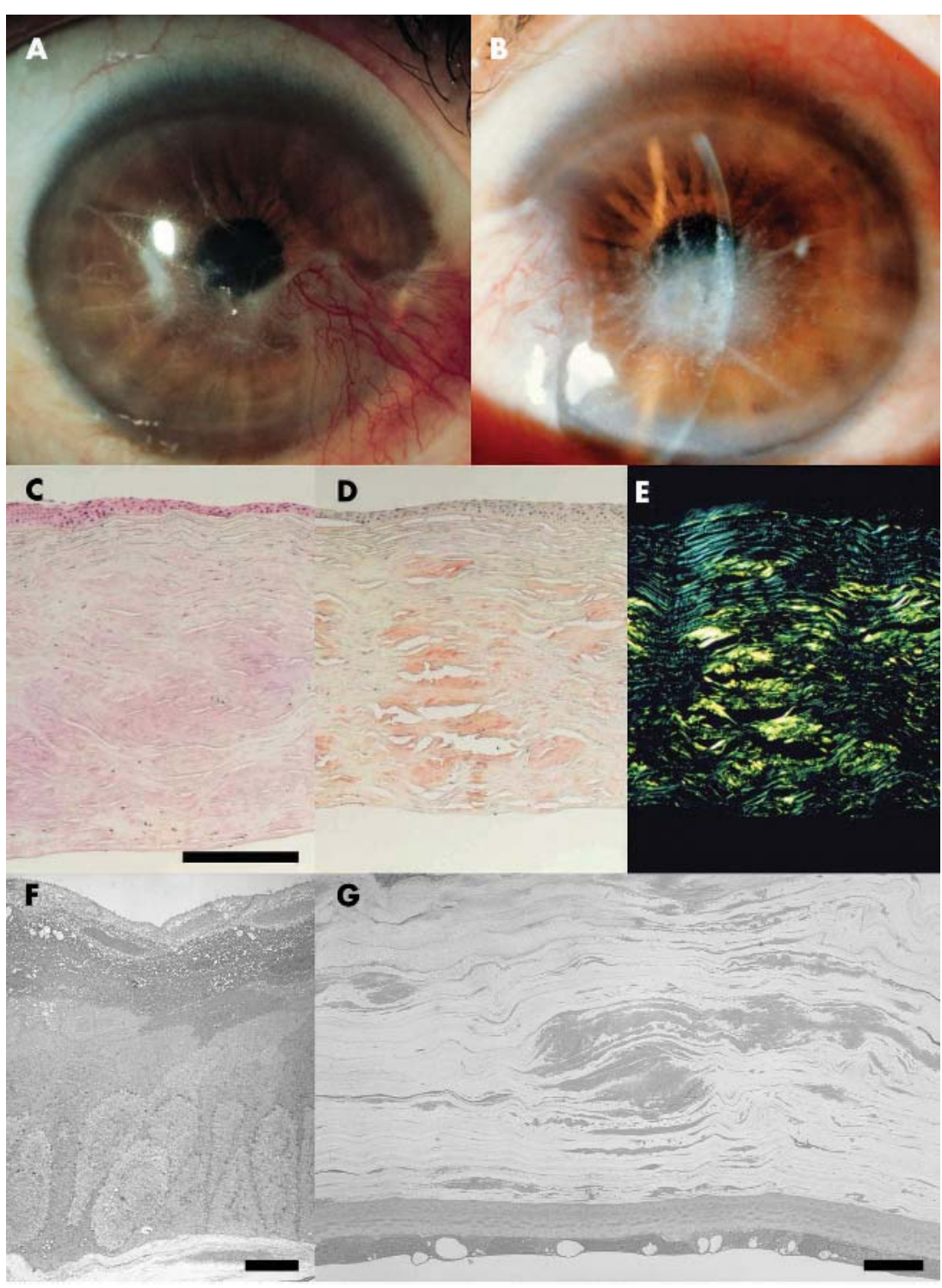

Figure 1 (A and B) Slit lamp photographs of the right and left eyes, respectively, of the patient. Light microscopic (C-E) and transmission electron microscopy (TEM) (F and G) analysis of the surgically removed corneal specimens of the patient. (C) Haematoxylin and eosin staining of the left cornea. (D) Congo red staining of the left cornea. (E) Polarised light microscopy of the left cornea. (F) TEM of the epithelial cell layer of the right cornea. (G) TEM of the stromal and endothelial cell layers of the right cornea. Scale bars: $250 \mu \mathrm{m}(\mathrm{C}-\mathrm{E})$ or $10 \mu \mathrm{m}$ (F and G).

suggested that his father had had LCD and that his younger sister had also developed this condition.

Slit lamp examination revealed a large region of opacity and thick lattice lines in the middle to deep portion of the corneal stroma in both eyes (fig IA and B). Given that his visual acuity in both eyes decreased to $20 / 40$, we performed penetrating keratoplasty on his left eye in December 2000 and on his right eye in May 2002. After the surgeries, his visual acuity improved to 20/25 in each eye and no recurrence has been observed to date.

Histological analysis revealed that the amorphous component of the middle to deep region of the stroma of both corneas stained with eosin (fig lC) and with Congo red (fig 1D). The detection of apple green dichroism by polarised light microscopy was also consistent with amyloid deposition in the middle to deep region of the corneal stroma (fig 1E). Although most of the epithelial cell layer, basement membrane, and hemidesmosomes of the right cornea appeared normal by transmission electron microscopy (TEM) (fig lF), a region was detected that seemed to be devoid of basal cells, basement membrane, and hemidesmosomes. TEM also revealed amyloid deposits in the middle to deep corneal stroma (fig lG), although Descemet's membrane and endothelial cells appeared normal.

After obtaining informed consent, we purified DNA from the white blood cells isolated from $10 \mathrm{ml}$ of the patient's blood. With appropriate primers, ${ }^{6}$ we amplified exons 4 and 12 of TGFBI by the polymerase chain reaction (PCR) and directly sequenced the products. We detected a homozygous point mutation, CTG $\rightarrow$ CGG (L527R), in codon 527 of TGFBI in the proband (fig 2). Point mutations were not detected in codons $124,518,544,546$, or 555 , mutations in

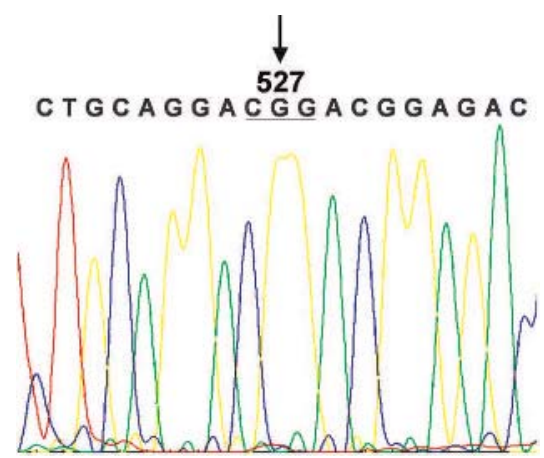

Figure 2 Molecular genetic analysis of TGFBI of the patient. Direct sequencing of PCR products corresponding to exon 12 of TGFBI. A homozygous $T \rightarrow G$ mutation (arrow) was detected at codon 527.

which have been associated with corneal dystrophies.

\section{Comment}

Fujiki $e$ e $a l^{2}$ reported that LCDIIIA in L527R heterozygotes is characterised by a late onset and mild clinical findings. Hirano et $a l^{7}$ reported that the condition caused by L527R heterozygosity was associated with amyloid deposition in the deep corneal stroma but not with corneal erosion. In contrast, LCD in our patient with a homozygous L527R mutation was characterised by onset in middle age, recurrent corneal erosion, and amyloid deposition in the middle to deep region of the stroma. The deposits in the proband showed right eye-left eye asymmetry in size and shape, as previously described for some L527R heterozygotes. ${ }^{2}$

In general, corneal dystrophies caused by homozygous point mutations in TGFBI are characterised by an earlier onset, more severe symptoms, and a higher frequency of recurrence after keratoplasty compared with those attributable to the corresponding heterozygous mutations. ${ }^{8-12}$ Our study and the two previous studies $^{27}$ of this mutation suggest that this is also the case for L527R. However, the difference in the findings of slit lamp examination between L527R heterozygotes and homozygotes with LCD appears to be less marked than that observed between R124H heterozygotes and homozygotes with Avellino corneal dystrophy. The reason for this discrepancy remains unclear at present.

\section{Acknowledgements}

This research was supported by grants from the Japan Society for the Promotion of Science (JSPS) and the Ministry of Education, Culture, Sports, Science, and Technology of Japan (to MI and TN).

\section{N Yamada, T-i Chikama, N Morishige,} R Yanai, T Nishida

Department of Biomolecular Recognition and Ophthalmology, Yamaguchi University School of Medicine, Ube City, Yamaguchi 755-8505, Japan

N Yamada, R Yanai, M Inui

Department of Pharmacology, Yamaguchi University School of Medicine, Ube City, Yamaguchi 755-8505, Japan

K Seki

Department of Ocular Pathophysiology, Yamaguchi University School of Medicine, Ube City, Yamaguchi 755-8505, Japan 
Correspondence to: Naoyuki Yamada, MD, PhD Department of Biomolecular Recognition and Ophthalmology, Yamaguchi University School of Medicine, 1-1-1 Minami Kogushi, Ube City, Yamaguchi 755-8505, Japan n.yamada@po.cc.yamaguchi-u.ac.jp

doi: $10.1136 /$ bjo. 2004.056168

Accepted for publication 1 November 2004

\section{References}

Yamamoto S, Okada M, Tsujikawa M, et al. A kerato-epithelin (betaig-h3) mutation in lattice corneal dystrophy type IIIA. Am J Hum Genet 1998:62:719-22.

2 Fujiki K, Hotta Y, Nakayasu K, et al. A new L527R mutation of the betalGH3 gene in patients with lattice corneal dystrophy with deep stromal opacities. Hum Genet 1998;103:286-9.

3 Mashima $Y$, Yamamoto $S$, Inove $Y$, et al. Association of autosomal dominantly inherited corneal dystrophies with BIGH3 gene mutations in Japan. Am J Ophthalmol 2000;130:516-17.

4 Dighiero $P$, Drunat $S$, Ellies $P$, et al. A new mutation (A 546T) of the betaig-h3 gene responsible for a French lattice corneal dystrophy type IIIA. Am J Ophthalmol 2000;129:248-51.

5 Munier FL, Frueh BE, Othenin-Girard $\mathrm{P}$, et al. $\mathrm{BIGH} 3$ mutation spectrum in corneal dystrophies. Invest Ophthalmol Vis Sci 2002;43:949-54.

6 Munier FL, Korvatska E, Djemai A, et al. Keratoepithelin mutations in four 5q31-linked corneal dystrophies. Nat Genet 1997;15:247-51.

7 Hirano K, Hotta Y, Nakamura M, et al. Late-onse form of lattice corneal dystrophy caused by Leu527Arg mutation of the TGFBI gene. Cornea 2001;20:525-9.

8 Mashima $Y$, Konishi M, Nakamura $Y$, et al. Severe form of juvenile corneal stromal dystrophy with homozygous $\mathrm{R} 124 \mathrm{H}$ mutation in the keratoepithelin gene in five Japanese patients. Br J Ophthalmol 1998;82:1280-4.

9 Okada M, Yamamoto S, Watanabe $\mathrm{H}$, et al. Granular corneal dystrophy with homozygous mutations in the kerato-epithelin gene. Am J Ophthalmol 1998;126:169-76.

10 Okada M, Yamamoto S, Inove Y, et al. Severe corneal dystrophy phenotype caused by homozygous R124H keratoepithelin mutations. Invest Ophthalmol Vis Sci 1998;39:1947-53.

11 Fujiki K, Hotta Y, Nakayasu K, et al. Homozygotic patient with betaig-h3 gene mutation in granular dystrophy. Cornea 1998;17:288-92.

12 Inove T, Watanabe H, Yamamoto S, et al. Different recurrence patterns after phototherapeutic keratectomy in the corneal dystrophy resulting from homozygous and heterozygous $\mathrm{R} 124 \mathrm{H}$ BIG-H3 mutation. Am J Ophthalmol 2001;132:255-7.

\section{Vitreous amyloidosis in alanine 71 transthyretin mutation}

Familial amyloid polyneuropathy (FAP) associated with mutations in the transthyretin (TTR) gene is the commonest form of hereditary amyloidosis. The incidence of vitreous opacities in FAP varies from 5.4\% to $35 \%,{ }^{12}$ but vitreous opacities as part of systemic amyloidosis are virtually pathognomonic of FAP. Hereditary non-neuropathic systemic amyloidosis is associated with mutations in the genes for lysosyme, apolipoprotein A-I, or fibrinogen A $\alpha$-chain. There are some 80 known mutations in TTR gene of which the methionine 30 variant is the most common. ${ }^{3}$ The rare alanine 71 (Ala 71) variant with vitreous opacities has been described in one family from France and another from Spain. ${ }^{45}$ We report a case of FAP Ala 71 without a family history of the disease who presented with a monocular inferior visual field defect and a corresponding vitreous opacity. Amyloid deposition was
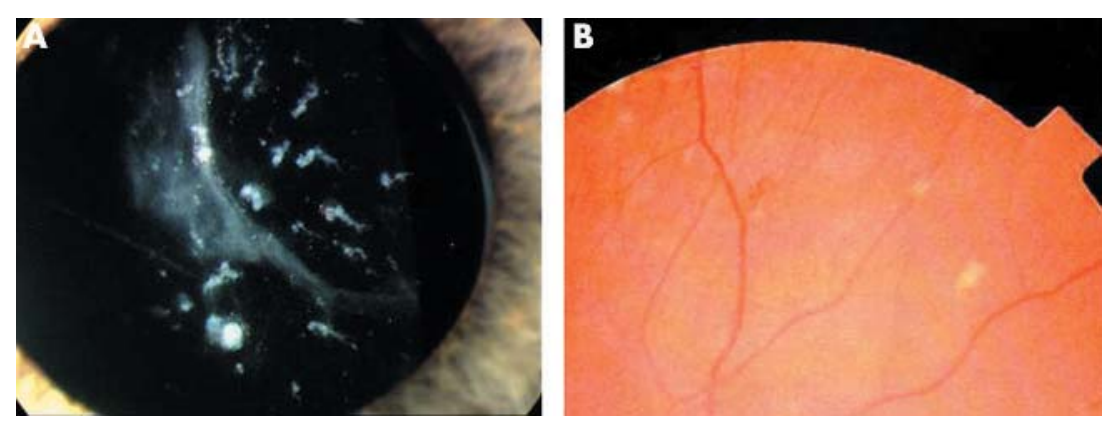

Figure 1 Dense nodular white deposits on the posterior capsule of the left eye were presumed to be of amyloid origin (A), and fundus periphery demonstrates intraretinal haemorrhages and perivascular white deposits (B)

subsequently diagnosed on vitreous and sural nerve biopsy.

\section{Case report}

A 46 year old woman presented with an inferior visual defect in her left eye. Ocular and systemic evaluation was normal including brain computed tomography scan. Eighteen months later, the patient developed bilateral floaters and visual loss in the left eye reducing her vision to 20/15 right and 20/30 left. Deposits of white "fluffy" material were noted on the posterior capsule of the left eye (fig lA) as well as bilateral "branching" vitreous opacities, peripheral retinal haemorrhages and perivascular sheathing (fig 1B). The left eye had a partial posterior vitreous detachment (PVD), a large vitreous floater and old inferior vitreous haemorrhage $(\mathrm{VH})$.

Vitreous opacification progressed and visual acuity was reduced to hand movement l year later. A full blood count, coagulation screen, and biochemical profile were normal. Creatinine clearance demonstrated a mild reduction in renal function and plasma cell dyscrasia was ruled out. Pars plana vitrectomy successfully cleared the vitreous debris, restoring vision to $20 / 20$ in the left eye. Eighteen months later the patient developed a right foot drop, progressive lower limb numbness, and numbness in both hands. A sural nerve biopsy established the diagnosis of amyloidosis and immunohistochemistry confirmed that TTR was the major protein constituent of the deposits. Cardiac involvement was demonstrated on echocardiography and renal involvement was confirmed by serum amyloid $\mathrm{P}$ (SAP) scintigraphy. Sequencing of her TTR gene confirmed that she was heterozygous for the amyloidogenic Ala 71 variant.

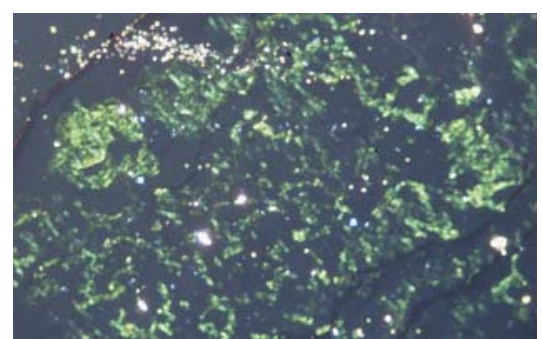

Figure 2 Apple-green birefringence with Congo red stain viewed with polarised microscopy of a vitreous biopsy confirming the presence of amyloid deposits.
The patient's right visual acuity deteriorated to $20 / 120$ because of increasing white vitreous opacities and nodular opacities on the anterior vitreous face. Right vitrectomy resulted in a return of visual acuity to 20/20. An undiluted right vitreous biopsy confirmed large amounts of amyloid of the TTR type (fig 2). The patient currently awaits orthoptic liver transplantation (OLT).

\section{Comment}

Vitreous opacification was initially attributed to old VH secondary to idiopathic retina vasculitis. Vitreous biopsy subsequently confirmed amyloid in the fellow eye. VH may occur secondary to vascular adventitial amyloid deposition or vitreous separation leading to a retinal tear, although vitreous opacities may be misinterpreted as Kantarjian and de Jong first reported vitreous amyloid in FAP. Amyloid of the vitreous body has been described as "glass wool, sheet-like veils or string of pearls white opacities," which differs from localised ocular amyloid in the orbit, lacrimal gland, conjunctiva, eyelids, sclera, and more specific forms in the cornea. Previous studies have reported abnormal conjunctival vessels, pupillary abnormalities, keratoconjunctivitis sicca, glaucoma, and vitreous opacities.

Almost all of the circulating TTR is produced in the liver and OLT can halt the progression of this disease and lead to clinical improvement. ${ }^{7}$ SAP scintigraphy is a method for identifying and quantitatively monitoring amyloid deposits in vivo, ${ }^{7}$ but this technique is not sensitive enough to monitor vitreous amyloid. Surprisingly, progressive vitreous amyloid deposition has been reported follow ing OLT, suggesting the TTR that forms vitreous amyloid may be produced locally. ${ }^{8}$ Previous reports have established that TTR has a widespread distribution in the eye but TTR mRNA has exclusively been located in the retinal pigment epithelium (RPE). ${ }^{9}$ Given that plasma TTR does not cross Bruch's membrane, it appears that ocular TTR is synthesised at least in part in the RPE, but the exact factors determining amyloid deposition are not understood.

Bilateral "branching" vitreous deposits of unknown aetiology should always raise the possibility of systemic amyloidosis. A relevan family history should be sought, but even in its absence, mutations of the TTR gene should be looked for.

H J Zambarakii, D G Charteris Moorfields Eye Hospital, Vitreoretinal Service London, UK 
W Ayliffe

Mayday University Hospital, Ophthalmology Department, London, UK

P J Luthert

Institute of Ophthalmology, Pathology Department London, UK

F Schon

Mayday University Hospital, Neurology Department London, UK

P N Hawkins

National Amyloidosis Centre, Department of Medicine, Royal Free and University College Medical School, London, UK

Correspondence to: $\mathrm{Dr} \mathrm{H}$ Zambarakji, Angiogenesis, Massachusetts Eye and Ear Infirmary, 325 Cambridge Street, Boston, MA 02114, USA hzambaraji@aol.com or

HZ@meei.harvard.edu

doi: 10.1136/bjo.2004.057554

Accepted for publication 20 October 2004

\section{References}

1 Ando E, Ando Y, Okamura R, et al. Ocular manifestations of familial amyloidotic polyneuropathy type I: long term follow up. Br J Ophthalmol 1997;81:295-8.

2 Koga T, Ando E, Hirata A, et al. Vitreous opacities and outcome of vitreous surgery in patients with familial amyloidotic polyneuropathy. Am J Ophthalmol 2003;135:188-93.

3 Connors LH, Richardson AM, Theberge R, et al. Tabulation of transthyretin (TTR) variants as of $1 /$ 1/2000. Amyloid 2000;7:54-69.

4 Benson MD, Turpin JC, Lucotte G, et al. A transthyretin variant (alanine 71) associated with familial amyloidotic polyneuropathy in a French family. J Med Genet 1993;30:120-2.

5 Almeida MR, Lopez-Andreu F, Munar-Ques M, et al. Transthyretin ALA 71: a new transthyretin variant in a Spanish family with familial amyloidotic polyneuropathy. Hum Mutat 1993:2:420-1.

6 Kantariian AD, de Jong RN. Familial primary amyloidosis with nervous system involvement. Neurology 1953;3:399-409.

7 Rydh A, Suhr O, Hietala SO, et al. Serum amyloid $\mathrm{P}$ component scintigraphy in familial amyloid polyneuropathy: regression of visceral amyloid following liver transplantation. Eur J Nuc Med 1998;25:709-13.

8 Munar-Ques M, Salva-Ladaria L, Mulet-Perera P, et al. Vitreous amyloidosis after liver transplantation in patients with familial amyloid polyneuropathy: ocular synthesis of mutant transthyretin. Amyloid 2000;7:266-9.

9 Cavallaro T, Martone RL, Dwork AJ, et al. The retinal pigment epithelium is the unique site of transthyretin synthesis in the rat eye. Invest Ophthalmol Vis Sci 1990;31:497-501.

\section{Multifocal electroretinogram demonstrated macular toxicity associated with ethambutol related optic neuropathy}

Ethambutol is an effective drug in the first line treatment for tuberculosis but its use may be associated with ocular toxicity. ${ }^{1}$ Toxic optic neuropathy is the most important ocular side effect and is related to the dose and duration of treatment. ${ }^{2}$ It is usually bilateral and both central and peripheral types of optic neuropathy have been described. The central type involves the papillomacular bundle and results in decreased visual acuity, caecocentral scotoma, and blue-yellow colour vision loss, whereas the peripheral type causes peripheral visual field loss, especially bitemporal defects with sparing of visual acuity and red-green colour vision impairment. ${ }^{3}$ In additional to the optic nerve toxicity, studies have also demonstrated that ethambutol may also be toxic at the retinal level. ${ }^{4-6}$ We report a patient with ethambutol related toxic optic neuropathy associated with bilateral macular toxicity as demonstrated by multifocal electroretinogram (mfERG). To our knowledge, evaluation of ethambutol related macular toxicity with mfERG has not been previously reported

\section{Case report}

A 45 year old man with pulmonary tuberculosis presented with a 3 week history of gradual bilateral visual loss. He has been on antituberculosis therapy with ethambutol $900 \mathrm{mg}$ (15 mg/kg/day), rifampicin $600 \mathrm{mg}$, isoniazid $300 \mathrm{mg}$, and pyrazinamide $2 \mathrm{~g}$ for 4 months. The baseline best corrected visual acuity (BCVA) before antituberculosis therapy was 20/30 in the right eye and 20/20 in the left eye. On presentation, the BCVA was 20/200 bilaterally without afferent pupillary defect. Colour vision testing showed red green dyschromatopsia. Dilated fundus examination revealed bilateral small splinter haemorrhages adjacent to the optic discs with mild left optic disc swelling (fig lA). Visual field examination showed bilateral central scotoma (fig lB). Magnetic resonance images (MRI) of the brain and orbits were normal. A diagnosis of toxic optic neuropathy due to ethambutol was made.

Since the optic discs and fundus changes were relatively subtle compared with the reduction in visual acuity and the central visual field defects and red-green dyschromatopsia were atypical, further investigations

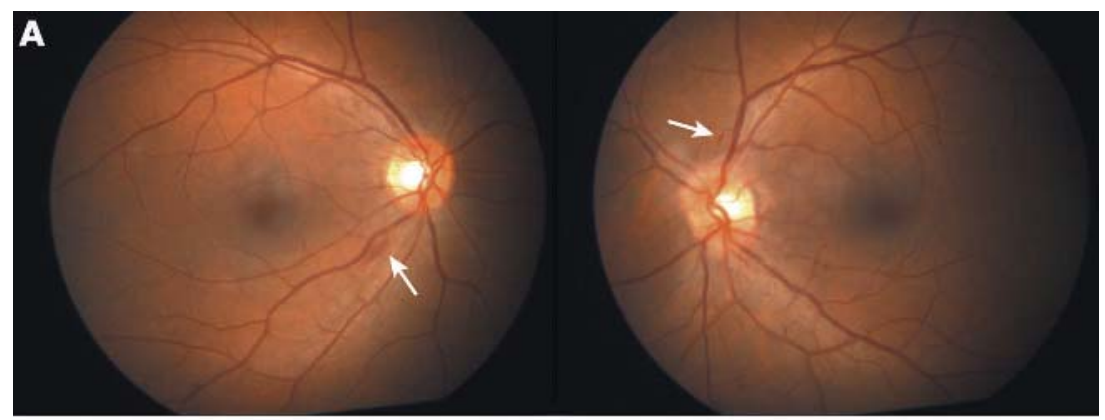

$\mathbf{B}$
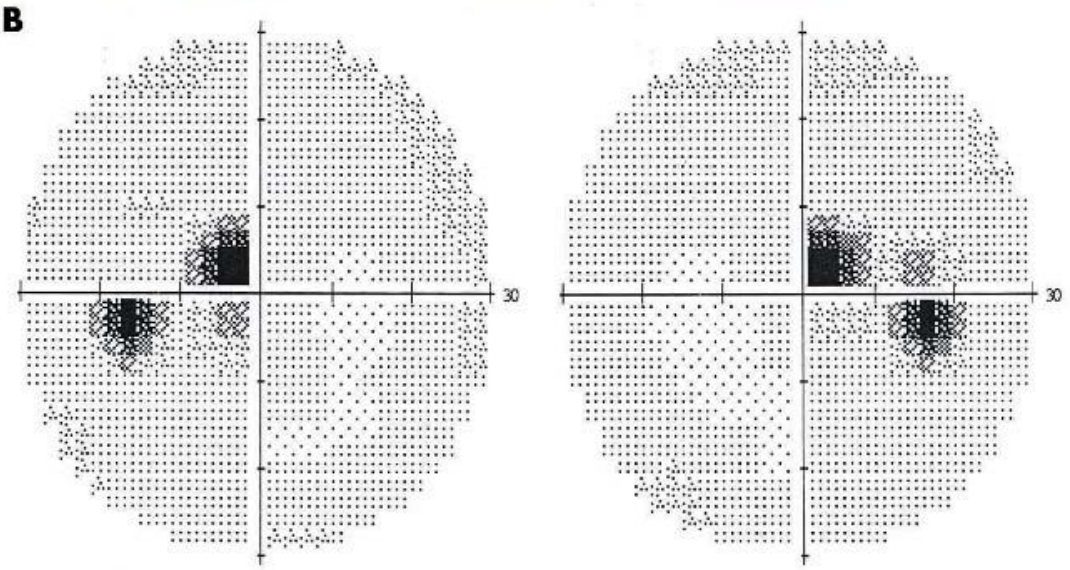

Figure 1 (A) Fundus photograph of the right (left) and left (right) eyes on presentation demonstrating bilateral splinter haemorrhages (arrows) with mild left optic disc swelling. (B) Automated visual field test showed central scotoma in the left (left) and right (right) eyes. were performed to evaluate potential associated retinal toxicity. These included electrooculogram (EOG), flash electroretinogram (ERG), pattern visual evoked potential (VEP), and mfERG. EOG and flash ERG of both eyes were normal. Pattern VEP showed and response for smaller checkers, with weak $1.8^{\circ}$. MfERG demonstrated bilateral general ised reduction in $\mathrm{Nl}$ and $\mathrm{Pl}$ retinal response amplitudes suggestive of toxic maculopathy (fig 2A).

In view of the optic neuropathy and maculopathy, all antituberculosis drugs were stopped after discussion with the physicians. The patient's BCVA gradually improved and 3 months later, his BCVA improved to 20/30 bilaterally. No abnormality was seen on fundus examination. Repeat mfERG recording showed recovery of the retinal responses in both eyes (fig 2B).

\section{Comment}

The exact mechanism of ethambutol induced toxic optic neuropathy is unclear but it may be due to retinal ganglion cells or bipolar cells toxicity at the retinal level. ${ }^{4-7}$ mfERG is a technique that allows objective assessment of (n) diseases ${ }^{8}$. In ERG which stimulates and measures the response of the entire retina, mfERG stimulates individual macular areas and measures responses from different retinal locations. It is useful in differentiating macular and optic nerve diseases as the mfERG in patients with optic nerve disease should be normal. ${ }^{9}{ }^{10}$ With the use of mfERG, we demonstrated that there was generalised reduction in MfERG responses at the macula and the areas of 

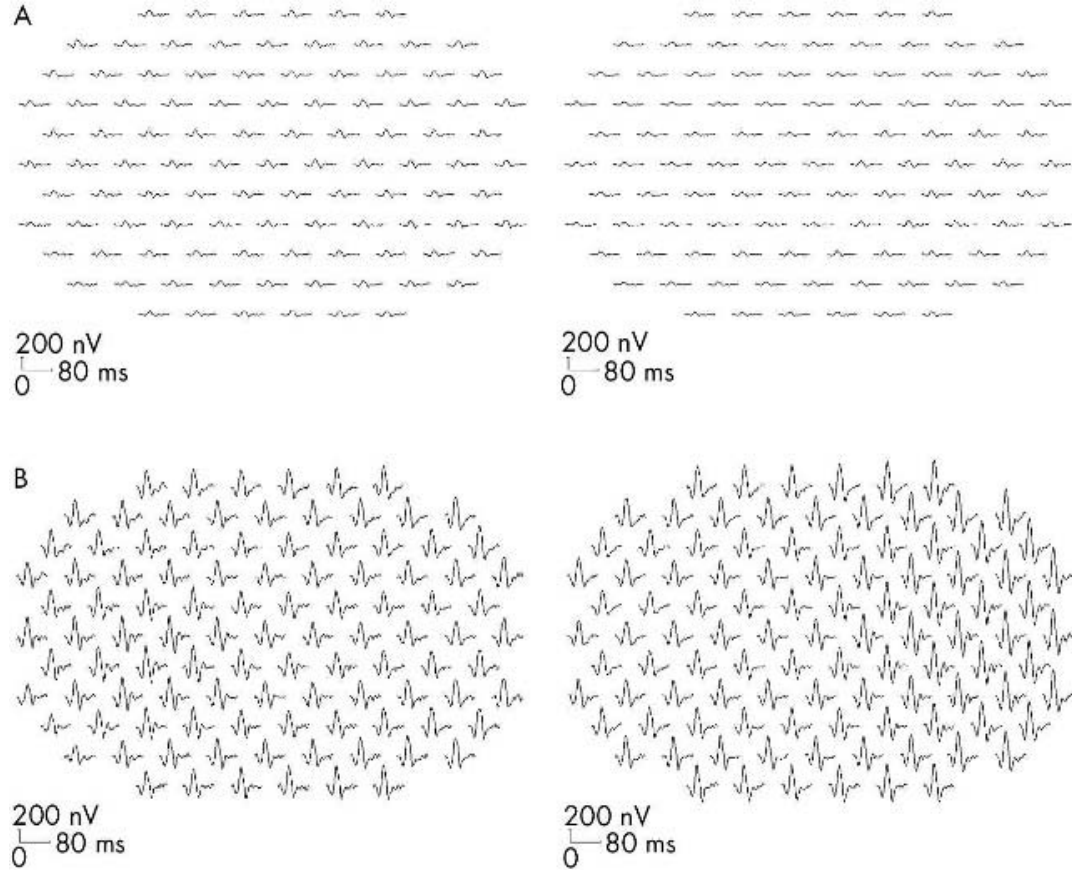

Figure 2 (A) Trace arrays of multifocal electroretinogram (mfERG) of the right (left) and left (right) eyes on presentation showing generalised reduction of retinal responses at the macula. (B) Trace arrays of mfERG of the right (left) and left (right) eyes 3 months after cessation of ethambutol demonstrating recovery and improvement of the $\mathrm{mfERG}$ responses.

abnormality were more extensive than the apparent localised visual field defects detected by automated perimetry. The abnormal mfERG suggests that in addition to toxic optic neuropathy, ethambutol may also cause macular toxicity at the neurosensory retinal level. The improvement in the patient's BCVA paralleled the improvement in mfERG responses and therefore mfERG may be a useful tool not only in diagnosis but in the serial assessments of ethambutol related ocular toxicity, particularly in patients with central visual loss.

T Y Y Lai, W-M Chan, D S C Lam Department of Ophthalmology and Visual Sciences, The Chinese University of Hong Kong, Hong Kong Eye Hospital, Hong Kong, People's Republic of China

E Lim

Department of Ophthalmology and Visual Sciences, The Chinese University of Hong Kong, Prince of Wales Hospital, Hong Kong, People's Republic of China

Correspondence to: Dr Wai-Man Chan, Department of Ophthalmology and Visual Sciences, The Chinese University of Hong Kong, 3/F, Hong Kong Eye Hospital, 147K Argyle Street, Kowloon, Hong Kong; cwm6373@netvigator.com

doi: 10.1136/bjo.2004.058099

Accepted for publication 20 October 2004

Financial support: nil.

Financial interest: nil.

\section{References}

1 Carr RE, Henkind P. Ocular manifestations of ethambutol. Toxic amblyopia after administration of an experimental antituberculous drug. Arch Ophthalmol 1962;67:566-71.

2 Leibold JE. The ocular toxicity of ethambutol and its relation to dose. Ann NY Acad Sci 1966; 135:904-9.
3 Schild H Fox. Raipd-onset reversible ocular toxicity from ethambutol therapy. Am J Med 1991;90:404-6.

4 Van Dijk BW, Spekreijse H. Ethambutol changes the color coding of carp retinal ganglion cells reversibly. Invest Ophthalmol Vis Sci 1983;24:128-33.

5 Kakisu Y, Adachi-Usami E, Mizota A. Pattern electroretinogram and visual evoked cortical potential in ethambutol optic neuropathy. Doc Ophthalmol 1987;67:327-34.

6 Nasemann J, Zrenner E, Riedel KG. Recovery after severe ethambutol intoxication: psychophysical and electrophysiological correlations. Doc Ophthalmol 1989:71:279-92.

7 Heng JE, Vorwerk CK, Lessell E, et al. Ethambutol is toxic to retinal ganglion cells via an excitotoxic pathway. Invest Ophthalmol Vis Sci 1999;40:190-6.

8 Sutter EE, Tran D. The field topography of ERG components in man: I. The photopic luminance response. Vis Res 1992;32:433-46.

9 Hood DC. Assessing retinal function with the multifocal technique. Prog Retin Eye Res 2000; 19:607-46.

10 Kretschmann U, Bock M, Gockeln R, et al. Clinical applications of multifocal electroretinography. Doc Ophthalmol 2000;100:99-113.

\section{Eye involvement mimicking scleritis in a patient with chronic lymphocytic leukaemia}

Acute leukaemia is known to affect the eye in a wide variety of ways and detailed postmortem examination will often reveal subclinical involvement. Ocular involvement by chronic leukaemia is much less common. ${ }^{1}$ We describe the clinical presentation, evaluation, and response to therapy of what is to our knowledge the first case of clinically significant scleral infiltration by chronic lymphocytic leukaemia mimicking scleritis.

\section{Case report}

An 87 year old man presented with a 2 week history of right eye redness, pain, and decreased vision. He complained of horizontal diplopia for 2 years, which had been corrected with prisms, but never investigated. Past medical history was notable only for chronic lymphocytic leukaemia (CLL), which was first diagnosed 17 years before presentation. He was treated intermittently with chlorambucil, but this was stopped as his white cell count had been stable at $30 \times$ $10^{9} / 1$ with minimal cervical and axillary lymphadenopathy. Vision was 6/18 right eye and 6/9 left eye. Intraocular pressure (IOP) was $18 \mathrm{~mm} \mathrm{Hg}$ in each eye. His vision was reduced by bilateral early cataracts and his right eye showed inferior scleral injection (fig 1A), a fibrinous anterior uveitis, and scattered posterior synechiae.

The patient was treated with topical corticosteroids and cycloplegics, but over the 3 weeks following presentation his vision dropped to 6/60 right eye because of choroidal effusions. He had restricted movement of the right eye, proptosis, and shallowing of the anterior chamber with an increase in IOP to $26 \mathrm{~mm} \mathrm{Hg}$. Ultrasound revealed thickening of the inferior sclera. A CT scan of the right orbit revealed a mass in the inferior fornix that was attached to the sclera (fig lB). Excision biopsy revealed patchy infiltration by foci of densely packed small lymphocytic cells, mainly around small blood vessels (fig 2A). Immunostaining showed a predominance of weakly stained CD20 positive B lymphocytes with scattered CD3 positive T cells consistent with CLL. Haematological analysis revealed a total white cell count of $49.1 \times 10^{9} / 1$. He received orbital radiotherapy $27.5 \mathrm{~Gy}$ in 11 fractions using $6 \mathrm{MV} x$ rays, completing the course 5 months after presentation. The inflammation, choroidal effusions, and orbital mass regressed completely but he was left with thinning of the inferior sclera (fig 2B). He was last seen 2 years after presentation with vision of 6/60 right eye but no evidence of active ocular involvement from his CLL. He refused cataract surgery.

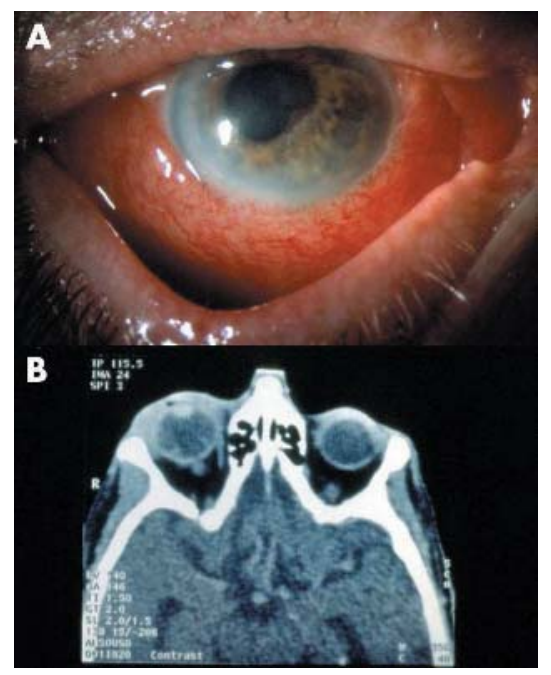

Figure 1 (A) Right eye showing diffuse injection of the inferior sclera. (B) Computed tomography (CT) scan of orbit showing a mass that was confluent with the sclera. 

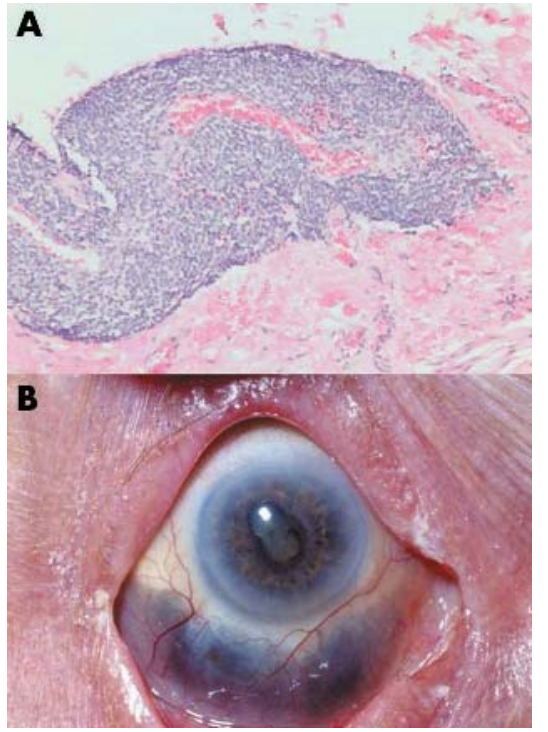

Figure 2 (A) Histopathology of scleral mass showing foci of densely packed small lymphocytic cells, mainly around small blood vessels (haematoxylin and eosin stain, original magnification $\times 200$ ). (B) Right eye following therapy showing thinning of the inferior sclera.

\section{Comment}

This patient presented with what at first appeared to be a scleritis with a secondary fibrinous anterior uveitis. He was 87 years old, which is an unusual age for a first presentation of either scleritis or anterior uveitis. His history of chronic diplopia and past medical history of CLL suggested a possible aetiology for these findings. It is uncommon for CLL to affect the eye in a clinically significant way ${ }^{2}$ although postmortem examination has shown relatively frequent subclinical involvement in between $30 \%$ and $90 \%$ of patients. ${ }^{3}$ Despite this, CLL has been described causing uveitis, glaucoma, iris infiltration, ${ }^{4}$ retinal detachments, conjunctival vascular changes, optic neuropathy, and orbital involvement as well as the more typical retinal haemorrhagic changes.

Episcleral and scleral infiltration is recognised in acute leukaemia and has been seen at necropsy in chronic leukaemia in $14 \%$ of cases in one series but this is rarely symptomatic.

In our patient the response to radiotherapy was encouraging and although his final acuity was not improved this was due in part to the progression of his cataracts. In summary, this is the first case we are aware of in which CLL had infiltrated the sclera of an eye to a sufficient degree that it simulated scleritis. The scleral mass regressed completely following radiotherapy although vision did not recover

B J L Burton Medical Retina Service, Moorfields Eye Hospital, City Road, London ECIV 2PD, UK

E T Cunningham Jr Department of Ophthalmology, New York University School of Medicine, New York, NY, USA

I A Cree Institute of Ophthalmology 11-43 Bath Street, London ECIV 9EL, UK
C E Pavesio

Medical Retina Service, Moorfields Eye Hospital, City Road London ECIV 2PD, UK

Correspondence to: $\mathrm{Mr} \mathrm{C}$ Pavesio, Moorfields Eye Hospital, City Road, London ECIV 2PD, UK; cpavesio@aol.com

doi: $10.1136 /$ bjo.2004.060152

Accepted for publication 16 November 2004

\section{References}

1 Allen RA Straatsma BR. Ocular involvement in leukemia and allied disorders. Arch Ophthalmol 1961;66:490-508

2 Buchan J, McKibbin M, Burton T. The prevalence of ocular disease in chronic lymphocytic leukaemia. Eye 2003;17:27-30.

3 Kincaid MC, Green WR. Ocular and orbital involvement in leukemia. Surv Ophthalmol 1983;27:211-32.

4 Martin B. Infiltration of the iris in chronic lymphatic leukaemia. Br J Ophthalmol 1968;52:781-5.

\section{Regression of choroidal metastases from breast carcinoma using aromatase inhibitors}

Breast cancer has become a leading health concern in the United States, accounting for $30 \%$ of all cancers among women. ${ }^{1}$ Oestrogen is an important hormone involved in the development and growth of breast tumours. ${ }^{2}$ It has been found that $60-70 \%$ of breast cancers have oestrogen receptors. ${ }^{2}$ Following tumour resection, receptor positive patients are commonly treated with hormone therapy such as tamoxifen. Tamoxifen is a competitive antagonist of oestrogen at its receptor site. It is most often used for postmenopausal patients with oestrogen receptor positive breast cancers. However, tamoxifen also has a partial oestrogen agonist effect which could be detrimental, because it can lead to increased risk for uterine cancer, thromboembolism, and treatment failure. ${ }^{3}$

The third generation aromatase inhibitors, including anostrozole, letrozole, and vorozole, have emerged as a new treatment for postmenopausal women with oestrogen receptor positive breast cancer. Their mechanism differs from that of tamoxifen as they minimise peripheral conversion of circulating androgen to oestrogen. ${ }^{3}$ We report one patient with choroidal metastasis from oestrogen receptor positive breast cancer who showed an excellent response to oral aromatase inhibitors.

\section{Case report}

A 66 year old woman presented in January 2003 with a 5 month history of flashing lights in the right eye. Her medical history revealed breast cancer, oestrogen receptor positive, treated with modified radical mastectomy and tamoxifen. In 1989, tamoxifen was stopped per the standard 5 year protocol. In October 2002, she developed metastases to the supraclavicular lymph nodes bone and was started on anastrozole.

On examination, visual acuity was 20/60 RE and 20/20 LE. Fundus examination revealed subretinal fluid overlying a solitary amelanotic choroidal metastasis measuring $12 \mathrm{~mm}$ in base and $3 \mathrm{~mm}$ in thickness (fig 1 ). The patient was continued on anastrozole. In
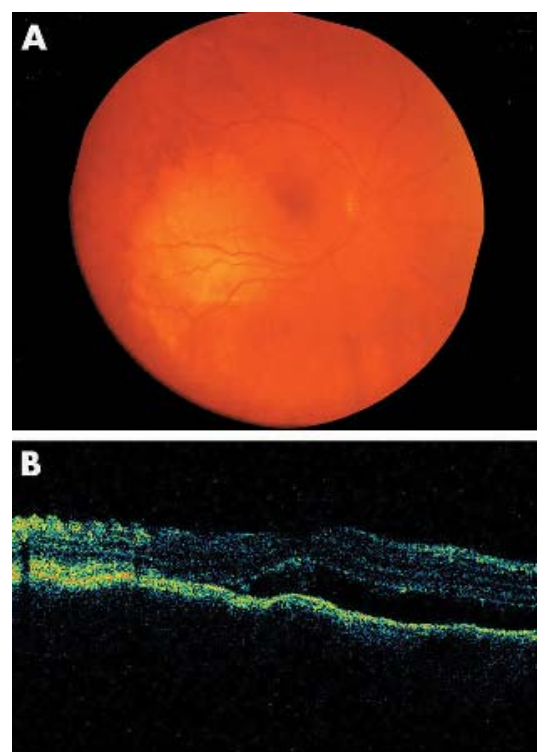

Figure 1 January 2003. (A) Active choroidal metastasis from breast carcinoma at first examination. (B) Optical coherence tomography showing subretinal fluid in the fovea.

September 2003, the choroidal metastasis completely regressed with resolution of subretinal fluid (fig 2). Her visual acuity remained 20/40 RE and 20/20 LE.

\section{Comment}

Uveal metastases are the most common intraocular malignancy. They typically affect the posterior choroids. ${ }^{4}$ The most common primary sites of cancer are from breast $(47 \%)$, lung $(21 \%)$, and gastrointestinal tract $(4 \%) .5$ Classically, choroidal metastases are yellow, plateau shaped, with secondary subretinal fluid. The treatment of choroidal metastases
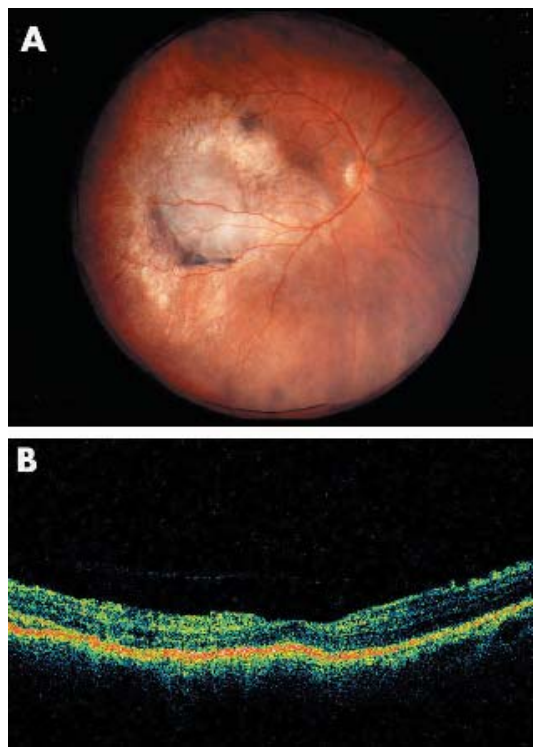

Figure 2 September 2003. (A) Following 9 months of anastrozole treatment, choroidal metastasis has regressed.(B) Optical coherence tomography following 9 months of anastrozole treatment showing resolution of subretinal fluid. 
depends on many factors including location, multiplicity, and activity of each tumour. ${ }^{6}$ Additionally, the projected visual outcome and underlying systemic control is important. In some cases, therapy is limited to the eye, especially if systemic metastases are absent or in remission. In these instances, external beam radiotherapy or plaque radiotherapy are employed. ${ }^{6}$ In most instances, however, the patient has systemically active metastatic disease so therapy is directed towards treatment of both the ocular and systemic disease using chemotherapy or hormone therapy. ${ }^{6}$

The most commonly employed hormonal treatment for breast cancer is tamoxifen. ${ }^{2}$ It is usually employed for postmenopausal patients with breast cancer who display oestrogen receptors. The effect of tamoxifen is due to its anti-oestrogenic activity, by competitive inhibition of oestrogen binding to oestrogen receptors. ${ }^{2}$ Tamoxifen inhibits the expression of oestrogen regulated genes including growth factors and angiogenic factors secreted by the tumour. Tamoxifen can also induce programmed cell death. Tamoxifen additionally has partial agonist effects, which could be beneficial because it prevents bone demineralisation in postmenopausal women. ${ }^{3}$ However, these oestrogenic effects are also associated with increased risks of uterine cancer, thromboembolism, and treatment failure. ${ }^{3}$

Tamoxifen is well tolerated by most patients with breast cancer and only $5 \%$ of patients note related menopausal symptoms, such as hot flashes and vaginal discharge. ${ }^{2}$ Retinopathy has been reported in women with high doses of tamoxifen, but not with conventional doses.

In postmenopausal women the main source of oestrogen is from peripheral conversion of adrenal androgen. New third generation aromatase inhibitors, including anastrozole (Arimidex, Zeneca) and letrozole (Femara, Novartis), act by preventing this conversion, thus lowering circulating oestrogen levels. Studies have shown that they are equal or superior to tamoxifen in clinically efficacy for metastatic breast carcinoma. ${ }^{3}$ They are well tolerated and have been shown to have important benefits over tamoxifen. Comparative trials indicate that anastrozole has similar adverse effects compared to tamoxifen. ${ }^{89}$ Our patient responded dramatically to aromatase inhibitors after failing to respond to tamoxifen. Based on this case and three other cases with a favourable response we anticipate that aromatase inhibitors could be a promising alternative for patients with choroidal metastases from oestrogen receptor positive breast carcinoma.

M E Manquez, C L Shields, E C Karatza, J A Shields

Oncology Service, Wills Eye Hospital, Thomas Jefferson University, Philadelphia, PA, USA

Correspondence to: Dr Maria Manquez, Oncology Service, Wills Eye Hospital, 840 Walnut Street, Philadelphia, PA 19107, USA memanquez@hotmail.com

doi: 10.1136/bjo.2004.061127

Accepted for publication 31 October 2004

Supported in part by the Pan American Ophthalmology Foundation and Retina Research Foundation (Dr ME Manquez), the Eye Tumor Research Foundation, Philadelphia, PA (Dr CL Shields) the Macula Foundation, New York, NY (Dr CL Shields), the Rosenthal Award of the Macula Sociely (Dr CL Shields), the Paul Kayser International Award of Merit in Retina Research, Houston, TX (Dr JA Shields).

\section{References}

1 Feig BW, Berger DH, Fuhrman GM. In: Breast cancer. The MD Anderson surgical oncology handbook. Philadelphia: Lippincott Williams and Wilkins, 2003:14

2 Osborne CK. Tamoxifen in the treatment of breast cancer. N Engl J Med 1998;339:1609-18.

3 Smith IE, Dowsett M. Aromatase inhibitors in breast cancer. N Engl J Med 2003;348:2431-42.

4 Shields JA, Shields CL. In: Metastatic tumor to the uvea and retina. Intraocular fumors. A text and atlas. Philadelphia: WB Saunders,

1992:278-320

5 Shields CL, Shields JA, Gross N, et al. Survey of 520 eyes with uveal metastases. Ophthalmology 1997:104:1265-76.

6 Shields CL. Plaque radiotherapy for the management of uveal metastasis. Curr Opin Ophthalmol 1998;9:31-7.

7 Demirci H, Shields CL, Chao AN, et al. Uveal metastasis from breast cancer in 264 patients. Ophthalmology 2003;136:264-71.

8 ATAC (Arimidex, Tamoxifen Alone or in Combination) Trialists' Group. Anastrozole alone or in combination with tamoxifen alone for adjuvant treatment of postmenopausal women with early breast cancer: first results of the ATAC randomized trial. Lancet 2002;359:2131-9.

9 Mouridsen H, Gershanovich M, Sun Y, et al. Superior efficacy of letrozole versus tamoxifen as first-line therapy for postmenopausal women with advanced breast cancer: results of a phase III study of the International Letrozole Breast Cancer Group. J Clin Oncol 2001;19:2596-606.

\section{"Fingertip" cryoprobe assisted orbital tumour extraction}

Cryoprobes are used to grasp, provide traction, and facilitate orbital tumour removal. ${ }^{1-4}$ Standard ophthalmic cryoprobe tips are typically rounded and offer small surface areas for cryo-adhesion. I describe a new spatulated cryotherapy probe with a large oval and uniform surface area for adhesion
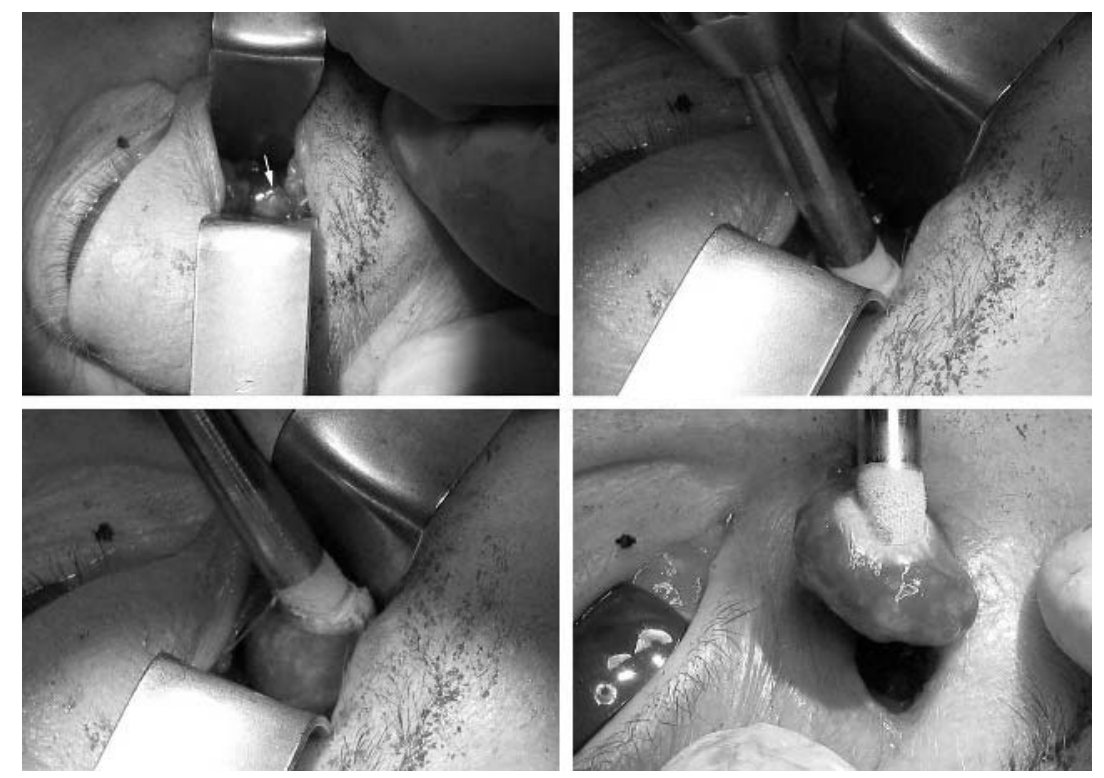

Figure 2 Top left, the tumour (arrow) can be seen deep within the superonasal orbit. Top right, the cryoprobe is introduced such that the spatulated tip is placed flush with the tumour and not touching adjacent orbital tissues. Bottom left, the tumour is delivered from the orbit utilising the cryoadhesion. Bottom right; the tumour is visualised outside the orbit. Note the freeze within the tissue with minimal ice formation on the shaft of the probe. 
portion of the frontal bone and optic nerve. Ultrasonography revealed intrinsic tumour vascularity (low flow). Cavernous haemangioma was suspected and an anterior orbitotomy performed. A combination of sharp and blunt dissection was required to expose the tumour. Then, a medium sized "Fingertip" cryoprobe was used to create an adhesion to the tumour's surface (fig 2). This relatively large cryo-adhesion allowed for easier handling of the tumour and improved traction (fig 2). The tumour remained adherent to the cryoprobe for 60 seconds after the nitrous oxide was discontinued. Freezing induced no change in the appearance of the tumour on gross examination. Histopathology revealed cavernous haemangioma. No clinical side effects (related to cryo-extraction) were noted.

\section{Comment}

Cryo-extraction works best with tumours and cysts that contain fluids (for example, blood, tears), as opposed to solid tumours and those containing lipid or keratin (for example, dermoid). Thus, cavernous haemangiomas are particularly good candidates because freezing occurs on both the tumour's surface (capsule) and within the tumour stroma. With a well formed fibrous capsule, the outer and inner ice balls provide a strong attachment to the probe.

The fingertip cryoprobes differ from previously available applicators in that they offer relatively large, spatulated tips. This allows for more homogeneous cryo-adhesion over a larger surface area. The larger surface area of application also increases tissue penetration, resulting in a stronger adhesion.

This study examines a new type of cryoprobe used to assist orbital surgery during tumour extraction. It was found capable of adhering to the tumour. Held by the surgeon, tumour traction could be induced with one hand, while the other hand wielded instruments used to sever residual tumour attachments. ${ }^{5} \quad$ Fingertip cryoprobes offer an alternative to current devices used for cryoextraction of orbital tumours.

Correspondence to: P T Finger, MD, The New York Eye Cancer Center, 115 East 61 st Street, New York City, NY 10021, USA; pfinger@eyecancer.com

doi: 10.1136/bjo.2004.055426

Accepted for publication 1 October 2004

The work is supported by The EyeCare Foundation, Inc and Research to Prevent. Blindness, New York, USA.

Dr Finger has no proprietary interest in the instrument described in this study.

\section{References}

1 Maroon JC, Onik G, Quigley MR, et al. Cryosurgery re-visited for the removal and destruction of brain, spinal and orbital tumours. Neurol Res 1992;14:294-302.

2 Henderson JW, Neault RW. The use of the cryoprobe in the removal of posterior orbital tumors. Ophthalmic Surg 1976;7:45-7.

3 Loewenstein A, Geyer O, Lazar M. Cavernous haemangioma of the orbit: treatment by transconjunctival cryoextraction. Eye 1993;7(Pt 4):597-8

4 Gdal-On M, Gelfand YA. Surgical outcome of transconjunctival cryosurgical extraction of orbital cavernous hemangioma. Ophthalmic Surg Lasers 1998;29:969-73.

5 Finger PT. "Finger-tip" cryoprobe assisted enucleation. Am J Ophthalmol, (in press).

\section{Congenital third nerve palsy, moyamoya disease and optic nerve head staphyloma}

The association of congenital optic nerve head anomalies, especially of the morning glory disc variety, with moyamoya disease is well recognised and has been described in a number of patients. ${ }^{1-4}$ To the best of our knowledge, the occurrence of a congenital third nerve palsy with moyamoya disease and a congenital optic nerve head anomaly has not been reported. We describe a patient who demonstrated the ipsilateral occurrence of these three congenital abnormalities, strengthening the association of congenital optic nerve and carotid abnormalities and suggesting a common underlying aetiology.

\section{Case report}

A 3 year old boy was examined because of left sided exotropia and blepharoptosis. His birth, developmental, and medical histories were unremarkable. Visual acuity was 20/25 right eye and 20/200 left eye. There was a near total ptosis of the left upper eyelid. There was a large exotropia and a small hypotropia of the left eye that he was unable to adduct, elevate, or infraduct. His right pupil reacted briskly to light, but the left pupil was dilated and sluggishly reactive to light. A left relative afferent pupillary defect was present. Fundus examination disclosed an anomalous left optic disc and peripapillary area, with what appeared to be an enlarged scleral opening and a staphylomatous defect around the optic papilla (fig 1). The rest of the retina was normal. The right fundus was normal.

A magnetic resonance imaging scan and magnetic resonance angiogram of the brain revealed an absence of the left intracranial carotid artery and its bifurcation into middle and anterior cerebral arteries. The lenticulostriate arteries were increased in size, consistent with moyamoya vessels (fig 2).

\section{Comment}

This patient has an optic nerve head malformation that appears to be most compatible with a peripapillary staphyloma or a variant of a morning glory disc anomaly (MGDA). MGDA is one of the cavitary optic disc malformations comprising a congenital, funnel-shaped excavation of the posterior peripapillary sclera that incorporates the optic disc. In addition to the anomalous retinal

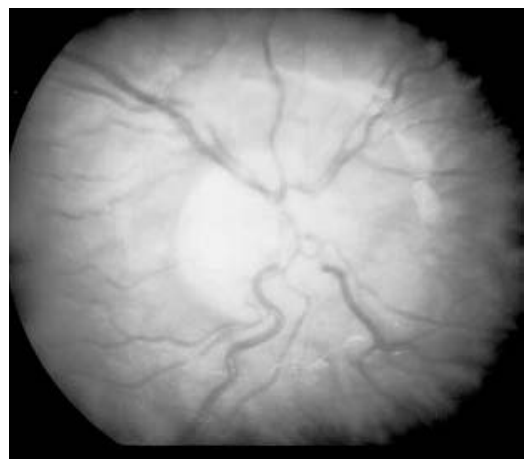

Figure 1 Enlarged optic nerve head scleral opening with radial exit of retinal blood vessels. Note staphylomatous appearance of peripapillary area.

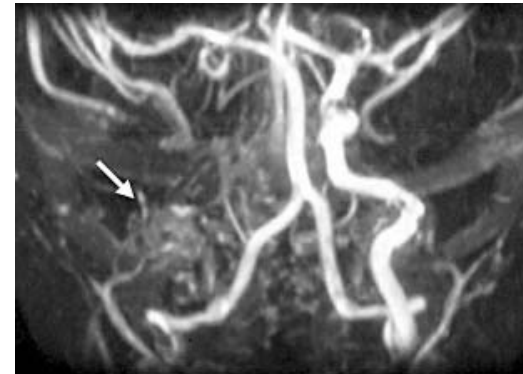

Figure 2 Cerebral angiogram shows total absence of left internal carotid artery (arrow) with increased size of lenticulostriate arteries compatible with moyamoya disease.

vasculature that distinguishes the MGDA from other excavated optic disc anomalies such as optic disc coloboma, the association of morning glory disc with carotid circulation anomalies, especially moyamoya disease has been reported. ${ }^{1-5}$ Moyamoya disease is a rare cerebrovascular disorder characterised by stenosis or occlusion of the distal internal carotid arteries. Progressive brain ischaemia triggers formation of a collateral vascular network in the basal ganglia region referred to as moyamoya (Japanese word for "cloud of smoke") vessels. The most common clinical features are transient ischaemic attacks and stroke in children, and intracranial haemorrhage in adults. Our patient appears to have an extreme form of this condition with total absence of the left internal carotid artery and collateral vessel formation typical of moyamoya disease.

Congenital third nerve palsy was once thought not to be associated with other neurological abnormalities. More recent studies have however revealed a high incidence of associated neurological deficits such as hemiparesis, seizures, hemianopia, and hydrocephalus. ${ }^{5}$ An association of congenital third nerve palsy with MGDA or peripapillary staphyloma has not been reported to the best of our knowledge. The third nerve palsy in the present case may be due to an ischaemic brainstem event or a developmental defect affecting the left third nerve nucleus.

The constellation of abnormalities in our patient confirms the association of moyamoya vessels with optic nerve head malformations such as MGDA or peripapillary staphyloma, and the possible neurological complications of this malformation complex, as evidenced by the third nerve palsy in this case. It is possible that an intracranial vascular dysgenesis may underlie some cases of the morning glory disc anomaly. We strongly recommend magnetic resonance angiography in conjunction with magnetic resonance imaging to identify carotid vascular anomalies in patients with MGDA or peripapillary staphylomas, especially in the presence of other neurological signs such as a congenital third nerve palsy.

K Sabti, B A Haii Al-Bahar Eye Center and the Department of Ophthalmology, University of Kuwait, Kuwait City, Kuwait

J-M Hwang

Department of Ophthalmology, Seoul Municipal Boramae Hospital, College of Medicine Seoul National University, Seoul, Korea 
J-M Hwang, E I Traboulsi

The Center for Genetic Eye Diseases, Cole Eye Institute, Cleveland, $\mathrm{OH}$, USA

J Reid

Department of Pediatric Radiology, The Cleveland Clinic Foundation, Cleveland, $\mathrm{OH}$, USA

Correspondence to: Elias I Traboulsi, MD, Center for Genetic Eye Disease, Cole Eye Institute, The Cleveland Clinic Foundation, i32, 9500 Euclid Avenue, Cleveland, OH 44195, USA; traboue@ccf.org

doi: 10.1136/bjo.2004.059246

Accepted for publication 1 November 2004

\section{References}

1 Hanson MR, Price RL, Rothner $A D$, et al. Developmental anomalies of the optic disc and carotid circulation. A new association. J Clin Neuroophthalmol 1985:5:3-8.

2 Massaro M, Thorarensen O, Liu GT, et al. Morning glory disc anomaly and moyamoya vessels. Arch Ophthalmol 1998;116:253-4.

3 Bakri SJ, Siker D, Masaryk T, et al. Ocular malformations, moyamoya disease, and midline cranial defects: a distinct syndrome. Am J Ophthalmol 1998;116:253-4.

4 Krishnan C, Roy A, Traboulsi E. Morning glory disk anomaly, choroidal coloboma, and congenital constrictive malformations of the internal carotid arteries (moyamoya disease). Ophthalmic Genet 2000;21:21-4

5 Tsaloumas MD, Willshaw HE. Congenital oculomotor palsy: associated neurological and ophthalmological findings. Eye 1997;11(P† 4):500-3

\section{Radial optic neurotomy for the treatment of acute functional impairment associated with optic nerve drusen}

Radial optic neurotomy was recently introduced as a treatment option in patients with central retinal vein occlusion. ${ }^{1}$ As described by Opremcak et al, ${ }^{1}$ central retinal vein occlusion might be related to increased pressure on the central retinal artery and vein as well as on optic nerve fibres in the confined space provided by the scleral ring. It was therefore suggested that a relaxation of the scleral outlet by a radial optic neurotomy might be an effective surgical treatment option. In the light of this information we hypothesised, that radial optic neurotomy may also be applicable in patients with visual field defects and deterioration of visual acuity associated with optic nerve drusen, where compression induced damage to optic nerve fibres is the underlying pathogenetic principle

\section{Case report}

A 27 year old female patient presented with extensive bilateral optic nerve drusen (fig lA). While visual acuity was light perception on the right eye over 4 years, she had experienced an acute and rapid deterioration of visual acuity from $20 / 32$ to $20 / 500$ and a progressive visual field loss within the past 6 weeks before she was seen in our institution (fig 1B). The progressive visual field defect had been documented carefully by the referring ophthalmologist. Besides the optic nerve drusen, there was no other ophthalmic pathology, no history of glaucoma, or any other relevant disease. With respect to the rapid functional deterioration we discussed radial optic neurotomy as a potential treatment option. Surgery was performed, after written informed consent, by one of the authors (AK) and consisted of standard three port pars plana vitrectomy and radial optic neurotomy. The incision was performed at the nasal edge of the optic disc in a radial fashion avoiding major retinal vessels. There were no intraoperative complications except a small haemorrhage at the incision site. The patient was then carefully followed postoperatively at 3 week intervals. Already at the first follow up visit a regression of the visual field defect and slight improvement of visual acuity was noted (fig 2A). At last presentation 10 weeks after surgery, the visual field defect was limited to the inferior nasal quadrant and visual acuity had improved to 20/32 (fig 2B). Funduscopy revealed a scar at the incision site, but no other pathologies (fig 2C).

\section{Comment}

Drusen of the optic nerve are the result of axonal degeneration of retinal ganglion cells and are composed of acellular concentric calcified laminations. ${ }^{3}$ While most cases are idiopathic, drusen have been described in association with several acquired conditions such as hypertensive retinopathy, vascular occlusion, optic atrophy, or chronic papilloedema. ${ }^{4}$ If drusen are located deep in the optic nerve, progressive enlargement may result in pressure induced atrophy of adjacent nerve fibres producing visual field constriction and anterior ischaemic optic neuropathy. ${ }^{2}$ Drusen have also been reported to occur predominantly in eyes with abnormally small optic discs. ${ }^{5}$ It had been previously hypothesised, ${ }^{1}$ that a radial incision at the nasal edge of the optic disc might result in a decompression of the scleral outlet and the associated neurovascular compression in patients with central retinal vein occlusion. Transferring this hypothesis to our patient, radial optic neurotomy seemed a reasonable therapeutic approach to us, as a relaxation or decompression of the scleral outlet might counteract the relevant pathogenetic principles of progressive visual field defect in optic nerve drusen. To our knowledge, radial optic neurotomy has not been used for the treat ment of progressive visual field defects in association with optic nerve drusen before We were very aware of the experimental character of this surgical intervention and carefully informed the patient before the operation. However, with respect to the rapid deterioration of visual acuity and progression of visual field constriction, there seemed to be no other treatment option available and the surgical intervention appeared justified.

The validity of the "scleral outlet compartment syndrome" concept in central retinal vein occlusion, as well as the effect of the radial neurotomy, has been questioned ${ }^{6}$ by Hayreh and is currently under discussion. However, the excellent functional outcome in this case may suggest that radial optic neurotomy potentially provides a relaxation and decompression of the optic nerve, allowing a recovery in conditions associated with pressure induced nerve fibre damage as in the case presented. In retinal vein occlusion, other additional mechanisms of action, such as the formation of chorioretinal shunts as a result of radial optic neurotomy, were described to contribute to visual recovery.

In summary, this case reports indicates that radial optic neurotomy might be considered in patients presenting with sudden visual loss and constriction of visual field in association with optic nerve drusen. We considered the surgical approach in this single case, as there was no other treatment option we could offer the patient that might restore vision.
A

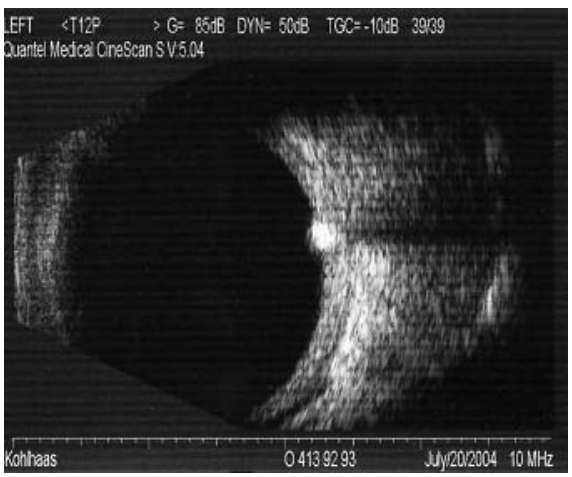

B

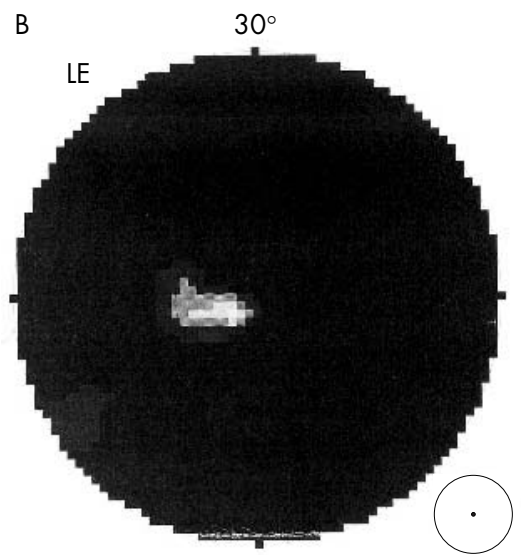

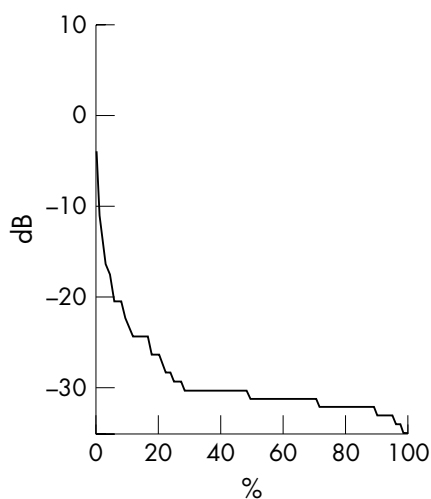

Figure 1 Highly reflective signal seen during B-scan echography at the optic nerve head of the left eye as typical for optic nerve drusen (A). The drusen were associated with a concentric visual field defect (B), visual acuity was 20/500. 


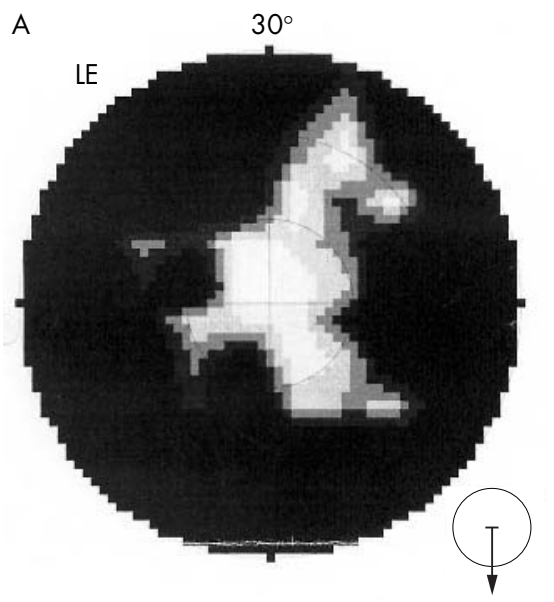

B

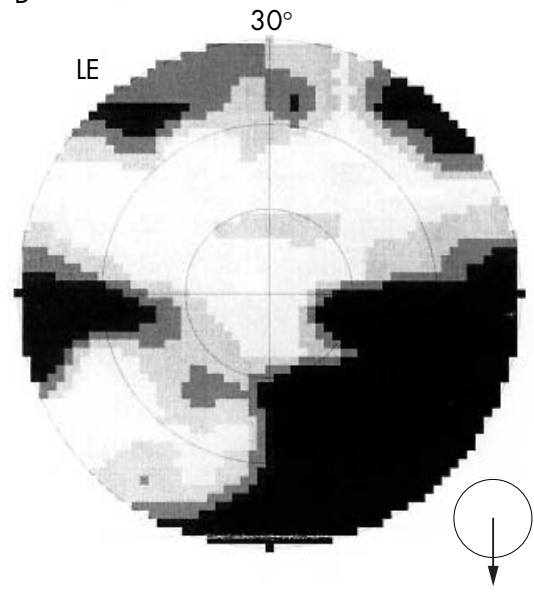

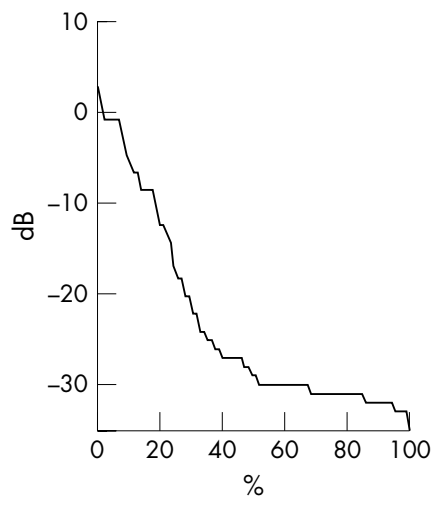

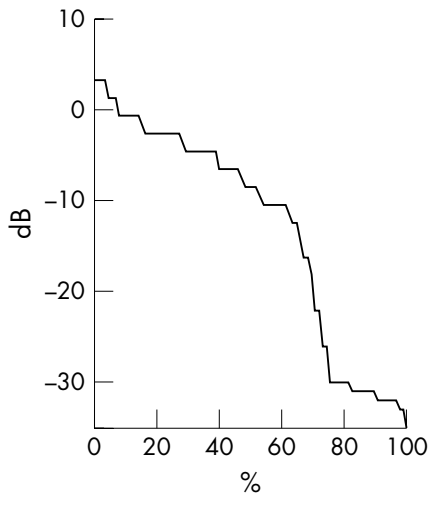

C

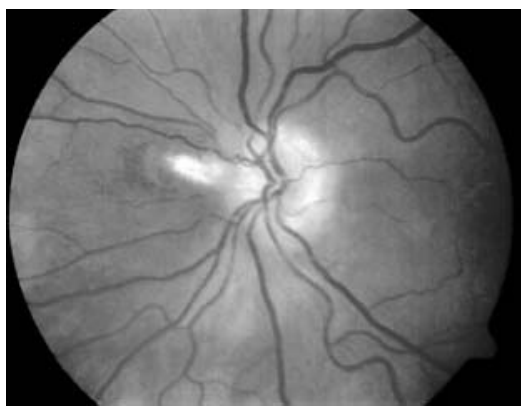

Figure 2 There was a regression of the visual field defect noted 3 weeks after radial optic neurotomy (A). Ten weeks postoperatively the visual field defect was predominantly limited to the lower nasal quadrant (B). Funduscopy revealed a scar at the incision site at the nasal rim of the optic disc (C).

\section{Haritoglou, S G Prieglinger, M Grueterich,} A Kampik Department of Ophthalmology, Ludwig-MaximiliansUniversity, Mathildenstrasse 8, 80336 Munich, Germany

\section{G K Kriegelstein}

Department of Ophthalmology, University of Cologne, Joseph-Stelzmann-Strasse 9, 50931 Cologne, Germany

Correspondence to: Christos Haritoglou, MD, Department of Ophthalmology, Ludwig-Maximilians-University, Mathildenstrasse 8, 80336 Munich, Germany Christos.Haritoglou@med.uni-muenchen.de

doi: $10.1136 /$ bjo. 2004.060335

Accepted for publication 2 November 2004

Financial interest: none.

\section{References}

1 Opremcak EM, Bruce RA, Lomeo MD, et al. Radial optic neurotomy for central retinal vein occlusion. A retrospective pilot study of 11 consecutive cases. Retina 2001 ;21:408-15.

2 Moody TA, Irvine AR, Cahn PH, et al. Sudden visual field constriction associated with optic disc drusen. J Clin Neuroophthalmol 1993;13:8-13.

3 Tso MOM. Pathology and pathogenesis of drusen of the optic nerve head. Ophthalmology 1981;88:1066-80.

4 Apple DJ, Rabb MF, Walsh PM. Congenital anomalies of the optic disc. Surv Ophthalmol 1982;27:3-41.
5 Jonas JB, Gusek G, Guggenmoos-Holzmann I, et al. Optic nerve head drusen associated with abnormally small optic discs. Int Ophthalmol 1987; 11:79-82

6 Hayreh SS. Radial optic neurotomy for central retinal vein occlusion. Retina 2002;22:374-7.

7 Garcia-Arumi J, Boixadera A, MartinezCastillo V, et al. Chorioretinal anastomosis after radial optic neurotomy for central retinal vein occlusion. Arch Ophthalmol 2003;121:1385-91.

\section{Early chorioretinal anastomosis in non-ischaemic CRVO: a randomised trial}

In non-ischaemic central retinal vein occlusion (niCRVO), the two principal determinants of final visual acuity are visual acuity and the presence of macular oedema at initial presentation. ${ }^{12}$ Data from the Central Vein Occlusion Study Group ${ }^{2}$ suggested that of patients with CRVO with an initial acuity better than 6/15, 65\% maintain this, whereas patients with presenting acuities between $6 / 15$ and $6 / 60,81 \%$ remain the same or get worse ( $19 \%$ improve to $6 / 12$ or better, $44 \%$ remain between $6 / 15$, and $6 / 60$ and $37 \%$ are worse than 6/60). Up to $34 \%$ of niCRVO may progress to the ischaemic variant with its attendant complications within 3 years. $^{2}$ However, in some studies $83 \%$ of indeterminate cases progress to ischaemic CRVO. The creation of a chorioretinal venous anastomosis (CRVA) improved visual acuity in some patients, decreased macular oedema, and reduced the incidence of progression to ischaemic CRVO. ${ }^{3-5}$ These important observations provide the basis for the study we carried out.

During the study period 11 patients (table 1) were enrolled according to the tria protocol (see appendix). All anastomoses were patent (on fluorescein angiography and if not repeated, see appendix). Mean patient age and mean pretreatment (T0) visual acuities, retinal thickness (by optical coherence tomography, OCT), and cyst height (OCT) did not differ between the two groups (table 1, fig 1). Factorial analysis of variance (ANOVA, Genstat) found that all three measures (acuity, retinal thickness, and cyst height, fig l) decreases significantly between T0 and 6 months, but only in the case of cyst height was there a significant difference between the laser treated and control groups $(F(1,9)=5.85, \mathrm{p}<0.05)$. The changes in retinal thickness and visual acuity were also greater in the treatment group but did not achieve significance $(F(1,9)=2.51$ and 1.15 , respectively, both $\mathrm{p}>0.1)$. Larger group sizes may have shown a significant effect.

The principal limitation of this trial is the small number of patients, because of the limited numbers of suitable patients within the study period. However, patients were randomised and well matched. The tria showed a trend towards better vision over 6 months in the laser treated group, although this failed to reach statistical significance. 

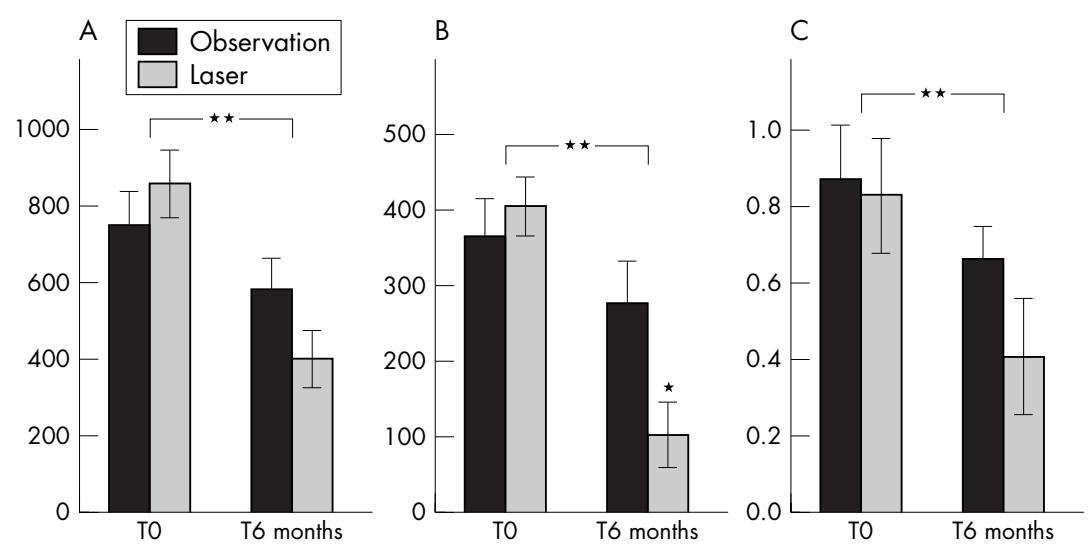

Figure 1 Data of retinal thickness (A), cyst height (B), and visual acuity (C) for patients randomised to laser chorioretinal venous anastomosis or observation. Visual acuity is measured on the ETDRS scale. Retinal thickness and cyst height were measured in pixels (1 pixel $=4 \mu \mathrm{m})$. Error bars show standard errors of means, obtained from factorial analysis of variance. Significant effects are identified as follows: ${ }^{*} \mathrm{p}<0.05 ;{ }^{* *} \mathrm{p}<0.01$.

It remains possible that a larger group of randomised patients would have produced statistically significant numbers. A multicentre trial would be required to achieve this. Another question arises regarding the longer term outcome in these patients. Again this should probably be considered in further studies. Cyst height might predict later visual acuity as there is evidence that it correlates with visual acuity outcome measures. ${ }^{67}$ It is therefore possible that longer follow up may have found a significant improvement in acuity. Retinal thickness predicts and precedes changes in visual acuity in diabetic retinopathy. ${ }^{8}$

If the size and duration of this trial had been increased, it is conceivable that results may have proved more informative. The fact that there was a significant effect on cyst height on OCT is, nevertheless, important and suggests that laser CRVA may reduce macular oedema in CRVO. The finding that improvements in visual acuity are recorded later than reduction in macular oedema ${ }^{8}$ in other studies implies that a significant effect on visual acuity might have been expected in these patients at a later time. We therefore recommend that this be taken into account in future studies.

\section{Acknowledgements}

We gratefully acknowledge the help of Professor Stephen Dunnett (Cardiff University) with statistical analysis of the data.

\section{R J Antcliff, E J Mayer, T H Williamson,} J S Shilling

Department of Ophthalmology, St Thomas's Hospital, Lambeth Palace Road, London El 7EH, UK

Correspondence to: Eric Mayer, University of Bristol, Clinical Sciences, Bristol Eye Hospital, Lower Maudlin Street, Bristol BS1 2LX, UK; e.mayer@bristol.ac.uk

doi: $10.1136 /$ bjo.2004.062539

Accepted for publication 14 January 2005

RJA was supported by the Lady Allerton Fund.

Competing or financial interests: none.

\section{Appendix}

The principal study end point was visual acuity at 6 months and resolution of macular oedema, the secondary end point was the identification of subgroups on optical coherence tomography (OCT 2000, Humphrey Instruments, USA) with a more favourable outcome including reduction of consecutive ischaemia. For entry into the study patients were required to: (1) be within 1 month of symptom onset; (2) have visual acuity of 6/24 or worse; (3) have good retinal perfusion (less than 10 disc diameters of non-perfusion); (4) have no relative afferent pupillary defect or neovascularisation; (5) have macular oedema confirmed by optical coherence tomography and fluorescein

Table 1 Comparison of randomised groups for early laser chorioretinal venous anastomosis versus observation in non-ischaemic CRVO (patient data are illustrated, visual acuities are given as ETDRS measures)

\begin{tabular}{llll}
\hline Parameter & Control & Laser & $t$ test \\
\hline Number of patients & 5 & 6 & \\
Female patients & 2 & 2 & \\
Left eyes & 5 & 3 & \\
Mean age & 76.4 & 68.8 & $1.278, p=0.117, \mathrm{NS}$ \\
Mean pretreatment acuity & 0.872 & 0.827 & $0.216, p=0.417, \mathrm{NS}$ \\
Mean 6 month acuity & 0.664 & 0.41 & $1.388, p=0.099, \mathrm{NS}$ \\
Mean change in acuity over 6 months & 0.208 & 0.417 & $-1.074, p=0.155, \mathrm{NS}$ \\
Mean change in retinal thickness on & 172.8 & 459.3. & $-1.583, p=0.074, \mathrm{NS}$ \\
OCT over 6 months & 87.6 & 302.6. & $-2.418, p=0.019^{*}$ \\
$\begin{array}{l}\text { Mean change in cyst height on } \\
\text { OCT over 6 months }\end{array}$ & & & \\
\hline
\end{tabular}

OCT, optical coherence tomography. fundus angiography (FFA). The following exclusion criteria were applied: diabetes with macular oedema in the fellow eye; inability to give informed consent; pregnancy; sensitivity to fluorescein; cloudy media sufficient to preclude adequate fundal photography; age less than 40 years.

Upon entry into the trial the following were performed: best corrected visual acuity (Snellen and ETDRS); fundus photography, and fluorescein angiography; OCT assessment of the macula. Patients were then randomised (envelope) to laser or observation groups. Laser anastomosis was performed with a $50 \mu \mathrm{m}$ spot size for 0.1 second with 1.5-2.5 W (maximum power possible) with an argon laser. The first shot was fired at the edge of the vein to rupture Bruch's membrane and the second shot at the edge of the vein to rupture the vein. A third shot with a YAG laser was applied $(3-5 \mathrm{~mJ})$ if required. Anastomotic sites were attempted in the following locations in order of preference-inferonasal, inferotemporal, or superonasal. All anastomoses were made at least 3 disc diameters from the optic disc. At 3-4 weeks repeat FFA was performed to determine anastomotic success: where no anastomosis was present this was repeated, until functioning.

Patients were reassessed at 1, 2, 3, 6, and 12 months and acuities and OCT recorded.

It was expected that approximately 40 patients would be required to show a $50 \%$ benefit.

Because of laser surgery it was not possible to mask the patient or observer as to which treatment had been carried out.

No adverse events were reported or noted in this series.

\section{References}

1 The Central Vein Occlusion Study. Baseline and early natural history report. Arch Ophthalmol 1993:111:1087-95.

2 The Central Vein Occlusion Study Group. Natural history and clinical management of central retinal vein occlusion. Arch Ophthalmol 1997:115:486-91.

3 McAllister IL, Constable IJ. Laser-induced chorioretinal venous anastomosis for treatment of nonischemic central retinal vein occlusion. Arch Ophthalmol 1995; 113:456-62.

4 McAllister IL, Douglas JP, Constable IJ, et al. Laser-induced chorioretinal venous anastomosis for nonischemic central retinal vein occlusion: evaluation of the complications and their risk factors. Am J Ophthalmol 1998;126 219-29.

5 McAllister IL, Vijayasekaran S, Yu DY, et al. Chorioretinal venous anastomoses: effect of different laser methods and energy in human eyes without vein occlusion. Graefes Arch Clin Exp Ophthalmol 1998;236: 174-81.

6 Antcliff RJ, Spalton DJ, Stanford MR, et al. Intravitreal triamcinolone for uveitic cystoid macular edema: an optical coherence tomography study. Ophthalmology 2001;108:765-72.

7 Beausencourt $\mathbf{E}$, Remky A, Elsner $A E$, et al. Infrared scanning laser tomography of macular cysts. Ophthalmology 2000; 107:375-85

8 Terasaki H, Kojima T, Niwa H, et al. Changes in focal macular electroretinograms and foveal thickness after vitrectomy for diabetic macular edema. Invest Ophthalmol Vis Sci 2003;44:4465-72 


\section{Informed consent and medical devices: the case of the contact lens}

It is estimated that 1.65 million people in the United Kingdom wear contact lenses, $97 \%$ of whom do so for refractive and cosmetic reasons. ${ }^{1}$ Contact lens wearers are at risk of sight threatening adverse events such as corneal ulceration, which may lead to scarring and visual loss. The risk of corneal ulceration is approximately 1 in 3000, 1 in 2000, and 1 in 500 patients per year for hard, soft daily wear, and soft continuous wear lenses, respectively. ${ }^{2-4}$

Informing individuals of the risks associated with contact lens wear, is important both for the wearer and person prescribing the contact lens. For consent to be legally valid it must be informed. This means that patients need information about the nature of an intervention, possible alternatives and, specifically, its benefits and risks. There is no reason why the principle of informed consent should not apply to the prescribing of a medical device such as a contact lens. We sought to determine the degree to which contact lens wearers have been informed of the risks associated with contact lens wear.

\section{Methods}

Following permission from the respective medical school deans, students at UK medical schools were contacted through electronic mail by their respective university faculties and asked if they wore or had worn contact lenses in the past year, to follow a link to a questionnaire (fig l) sited on the world wide web.

\section{Results}

In all, 560 replies were received (table 1); $21 \%$ of students had received some verbal information regarding the risk of corneal ulceration and $1 \%$ had received this in written form. Only $2 \%$ had been told of all of the four stated risks; $12-14 \%$ of respondents were aware of the risk of corneal ulceration associated with soft daily, continuous, and hard contact lens wear. Of those who knew of this risk $41 \%, 37 \%$, and $58 \%$ underestimated the respective morbidity. Only $23 \%$ of those who think that they had been given sufficient advice knew of the risk of corneal ulceration. Thirteen per cent of students reported the development of one or more complications associated with their contact lens wear. Eight per cent of students stated that had they been aware of the risks stated in the questionnaire they would not have chosen to wear contact lenses. $87 \%$ of students would still have chosen to wear a contact lens after reading the associated risks.

\section{Comment}

Contact lenses are worn by millions of people worldwide. Although the risk of a complication is small, it constitutes a significant public health problem when applied to a large population. Individually it is necessary to balance this risk against the benefits offered by contact lens wear. This can only be achieved with the provision of appropriate information.

Although there are limitations with this type of survey, it would appear that the majority of contact lens wearers, approximately $80 \%$ (98\% for all four of the stated
Q1 For how long have you been wearing contact lenses?

1-5 years

5-10 years

$>10$ years

Q2 Has your contact lens practitioner ever told you of the following risks which are associated with contact lens wear?

Corneal ulcers with loss of vision

Blood vessel formation on the cornea

Corneal scarring with loss of vision

Drooping of the upper eye lid (ptosis)

Yes/No

Yes/No

Yes/No

Yes/No

Q3 Has your contact lens practitioner ever given you written information on the following risks which are associated with contact lens wear? Corneal ulcers with loss of vision Blood vessel formation on the cornea

Yes/No Corneal scarring with loss of vision Drooping of the upper eye lid (ptosis)

Yes/No

Yes/No

Q4 Were you offered a choice of contact lens type by your contact lens practitioner?

Yes/No

Q5 Do you receive your contact lenses from hospital (Yes/No) or high street (Yes/No) contact lens practitioner?

Q6 Do you feel that the information given to you by your contact lens practitioner was sufficient?

Yes/No/Unsure

Q7 On a scale from one to five, with one being very well and five being not at all, how well do you think you understood the information you were given by your contact lens practitioner?

Q8 Have you ever developed any of the following problems? Corneal ulcers

Blood vessel formation on the cornea

Corneal scarring

Drooping of the upper eye lid (ptosis)

1-5

Yes/No

Yes/No

Yes/No

Yes/No

Q9 The estimated risk of corneal ulceration associated with contact lens wear is stated below. Please indicate if you were aware of these given risks. If the answer is yes please select whether you thought the risk was greater than stated, less than stated or the same as stated.

$\begin{array}{llllll} & \text { Risk } & \begin{array}{l}\text { Aware of } \\ \text { risk? }\end{array} & \begin{array}{l}\text { Greater } \\ \text { than }\end{array} & \text { Less than } & \text { Same as } \\ \text { Soft daily wear lenses } & 1 / 2000 & \begin{array}{l}\text { Yes/No } \\ \text { Yes/No }\end{array} & \begin{array}{l}\text { Yes/No } \\ \text { Yes/No }\end{array} \\ \begin{array}{lll}\text { Soft continuous wear lenses } \\ \text { Hard lenses }\end{array} & 1 / 500 & \begin{array}{l}\text { Yes/No } \\ \text { Yes/No }\end{array} & \begin{array}{l}\text { Yes/No } \\ \text { Yes/No }\end{array} \\ \text { Hes/No } & \text { Yes/No } / 3000 & \begin{array}{l}\text { Yes/No } / \text { Yes/No } \\ \text { Yes }\end{array}\end{array}$

Q10 If you were not aware of these risks, would you still have worn contact lenses had you be given the above information?

Yes/No

Figure 1 Questionnaire.

risks) of students surveyed, were unaware of the risks to which they were exposed.

It is important that potential contact lens wearers are fully informed to allow the best choice of refractive correction for them. Consumer comparison between the potential adverse effects of various contact lens types and wear patterns is important in this decision. This is emphasised here as $8 \%$ of those involved in the study would not have chosen to wear contact lenses had they known of the associated risks.

Although there is no legal stipulation regarding the provision of information when prescribing a contact lens, providing information and obtaining informed consent forms part of good clinical practice. Although written consent is not evidence that informed discussion has taken place, written agreements can be used to provide a basis for the process.

We would recommend that informed consent be obtained when prescribing contact lenses and that this should be done with the aid of written information. Concern over the associated risks should not deter people from the benefits of contact lens wear but they are an important consideration and consumers have a right to be informed.
A Roberts, A E Kaye, R A Kaye, K Tu, S B Kaye St Paul's Eye Unit, $8 Z$ Link, Royal Liverpool University Hospital, Prescot Street, Liverpool L7 8XP, UK

Correspondence to: Stephen B Kaye, St Paul's Eye Unit, $8 Z$ Link, Royal Liverpool University Hospital, Prescot Street, Liverpool L7 8XP, UK; Stephen.kaye@ rlbuht.nhs.uk

doi: 10.1136/bjo.2004.062315

Accepted for publication 16 November 2004

\section{References}

1 Schein OD, Glynn RJ, Poggio EC, et al. The relative risk of ulcerative keratitis among users of daily wear and extended wear contact lenses. N Engl J Med 1989:321:773-8.

2 MacRae S, Herman C, Stulting RD. Corneal ulcer and adverse reaction rates in premarket contact lens studies. Am J Ophthalmo $1991 ; 111: 457-65$.

3 Poggio EC, Glynn RJ, Schien OD, et al. The incidence of ulcerative keratitis among users of daily wear and extended wear soft contact lenses. N Engl J Med 1989;321:779-83.

4 Cheng $\mathrm{KH}$, Leung SL, Hoekman HW, et al. Incidence of contact lens associated microbial keratitis and its related morbidity. Lancet 1999;354:181-5. 
Table 1 Survey of 560 medical students who are contact lens wearers. All results are in percentages

\begin{tabular}{|c|c|c|c|}
\hline \multicolumn{4}{|c|}{ Provision of information versus reported complications (\%) } \\
\hline Risk & Verbal & Written & Complications* \\
\hline Ulcer & 18 & 4 & 3 \\
\hline BV & 22 & 4 & 8 \\
\hline Scar & 21 & 4 & 4 \\
\hline Ptosis & 4 & 3 & 1 \\
\hline \multicolumn{4}{|c|}{ Reported awareness of risk of corneal ulceration } \\
\hline SDL & Aware & Unaware & \\
\hline Greater & 18 & 17 & \\
\hline Same & 41 & 18 & \\
\hline Less & 41 & 65 & \\
\hline SCL & Aware & Unaware & \\
\hline Greater & 23 & 21 & \\
\hline Same & 40 & 7 & \\
\hline Less & 37 & 72 & \\
\hline HGP & Aware & Unaware & \\
\hline Greater & 40 & 29 & \\
\hline Same & 2 & 0 & \\
\hline Less & 58 & 71 & \\
\hline \multicolumn{4}{|c|}{ Knowledge of risk of corneal ulcer versus adequacy of advice } \\
\hline & All & Adequate advice & Inadequate advice \\
\hline SDL & 14 & 23 & 21 \\
\hline $\mathrm{SCL}$ & 14 & 24 & 22 \\
\hline & 12 & 23 & 17 \\
\hline \multicolumn{4}{|c|}{ Percentage (\%) who would wear contact lens with knowledge of risks } \\
\hline Yes & 87 & & \\
\hline No & 8 & & \\
\hline
\end{tabular}

*Actual complications reported by students. Blood vessel formation on the cornea (BV). Soft disposable (SDL), soft contact lens (SCL), rigid gas permeable (RGP), or hard contact lens (HGP).

\section{Multiple use of single use solutions: a dangerous practice}

I am writing to raise an issue for routine ophthalmic and optometry practice that contravenes the principles of clinical governance and generates an avoidable infection risk to patients

My concern relates to single use preservative-free ophthalmic solutions, particularly those used for diagnostic purposes in hospital eye clinics and optometry practices. The regulatory guidelines ${ }^{1}$ and the updated guidance note from the Royal Pharmaceutical Society (www.rpsgb.org.uk/pdfs/ophprepguid. pdf) specify that products designed and licensed for single use must be discarded immediately after use.

In practice, however, a recent study ${ }^{2}$ has documented the routine misuse of single use solutions (Minims), generating an unnecessary risk of infection transferral between patients. Single use solutions used multiple times were shown to have a significantly higher rate of contamination than those that were used once then discarded $(45 \%$ versus $4 \%$ ). An associated audit (Qureshi MA, personal communication, 2004) of hospitals in the London region found that $86 \%$ of the ophthalmologists surveyed admitted to multiple uses of single use solutions between different patients.

The study demonstrated the principle that single use products could act as a vehicle for transmission of micro-organisms present on the practitioner's hands or in the lid flora, if shared between patients. We know that gloves are typically not worn in outpatient ophthalmic practice, and that hands are not always washed between each and every patient. We note that even though the eye drop unit itself does not touch the patient's eye or surrounding area, the practitioner often does touch the patient with his/her hands. Furthermore, the potential exists for infection transfer without physical contact. Aside from the risk of infecting the patient, the efficacy of the medication can be compromised in the event of contamination.

My own experience of ophthalmologists and hospital pharmacists indicates that in some cases the interpretation of the term "immediately" has been stretched beyond the simple instruction to "use once and discard." For example, some published papers (Marchese et $a l^{3}$ ) refer to a, now outdated, EMEA instruction to use such products within three hours of opening. The EMEA has since replaced this with an updated guideline $e^{4}$ indicating that the usage time after opening is dependent on the nature of the product, yet this still encourages the user to retain the product for multiple use after first opening.

Clinical governance requires that risks and hazards to patients be reduced to as low a level as possible. Single use products are available for this purpose and should be used as intended and as licensed for one patient only. Repeated use in the same patient over a limited period up to 24 hours after first opening the product may be possible, depending on the product, and at the discretion of the clinician who must ensure that the product is not used on any other patient. I urge your readers to take action as necessary to ensure compliance.

Correspondence to: D V Seal, MD, Applied Vision Research Centre, City University, Northampton Square, London ECIV OHB, UK; davidseal@blueyonder.co.uk

doi: 10.1136/bjo.2004.064535

Accepted for publication 4 January 2005

\section{References}

1 Health Service Circular HSC (IS) 122. London: DHSS, 1975.

2 Wong R, Qureshi MA, Rowe C, et al. Dangers of multiple use of Minims preservative free eye drops in applanation tonometry. 5th International Symposium on Ocular Pharmacology and Therapeutics (ISOPT), 2004.
3 Marchese A, Bozzolasco M, Gualco L, et al. Evaluation of spontaneous contamination of ocular medications. Chemotherapy 2001;47:304-8.

4 EMEA. Maximum shelf life for sterile products for human use after first opening or following reconstitution. CPMP/QWP/159/96corr, 1998 (www.emea.eu.int/pdfs/human/qwp/ 015996en.pdf).

\section{MAILBOX}

\section{Drug induced autoenucleation with resultant chiasmal damage}

I read with interest the report of Tuwir et al ${ }^{1}$ of a case of autoenucleation with chiasmal damage. They note the rarity of this dramatic self mutilating event, and state that there have been only two other reports of contralateral field defect from chiasmal injury in instances of autoenucleation.

There have, however, been a number of other reports of temporal field defect in the remaining eye following either self enucleation or traumatic enucleation by an assailant. The consequences are the same regardless of who removes the eye. One of the two reports Tuwir et al cite, Parmar et al, ${ }^{2}$ was of a young man whose eye was digitally enucleated by an assailant.

In their 1984 review article on autoenucleation, Krauss $e t$ al $^{3}$ reported a 29 year old woman who had enucleated her right globe causing a complete temporal hemianopia in her remaining eye. They also reviewed the medical literature and found 19 cases of bilateral self enucleation (or of self mutilation so severe as to require enucleation) and 31 cases of unilateral self enucleation. Of the 18 eyes where the length of the attached optic nerve was noted, six occurred at or near the chiasm. They also found seven cases of traumatic avulsion of one eye resulting in a temporal hemianopia in the remaining eye.

In 1996, Arkin et al ${ }^{4}$ reported a 25 year old man with a temporal defect in his right eye after his left eye was pulled out by an assailant. A magnetic resonance image confirmed damage to the chiasm. And in 2002, Dilly and Imes $^{5}$ reported a 54 year old schizophrenic man who removed his blind left eye causing a complete temporal hemianopia in his remaining right eye. His MRI showed avulsion of the lateral portion of his chiasm.

It appears that even though acts of traumatic enucleation are rare, associated chiasmal injury is common.

Correspondence to: Richard K Imes, CPMC, USA; rimes56418@aol.com

doi: 10.1136/bjo.2005.068502

Accepted for publication 8 February 2005

\section{References}

1 Tuwir I, Chako E, Brosnahan D, et al. Drug induced autoenucleation with resultant chiasmal damage. Br J Ophthalmol 2005;89:121.

2 Parmar B, Edmunds B, Plant G. Traumatic enucleation with chiasmal damage: magnetic resonance image findings and response to steroids. Br J Ophthalmol 2002;86:1317-18.

3 Krauss H, Yee R, Foos R. Autoenucleation. Surv Ophthalmol 1984;29:179-87.

4 Arkin M, Rubin P, Bilyk J, et al. Anterior chiasmal optic nerve avulsion. AJNR 1996;17:1777-81.

5 Dilly J, Imes R. Autoenucleation of a blind eye. J Neuro-ophthalmol 2001;21:30-1. 


\section{P-glycoprotein expression in retinoblastoma}

We have read with interest the paper by Krishnakumar et al. ${ }^{1}$ We have studied 18 children with retinoblastoma using immunohistochemical detection of P-glycoprotein by the mouse monoclonal antibody HYB-24l in frozen section tumour samples. Like Krishnakumar et al, we found limited expression of P-glycoprotein in retinoblastoma cells since only four of the 18 samples were positive. However, six of 18 samples had Pglycoprotein positivity in tumour associated endothelial cells. Contrary to Krishnakumar et al, we had the opportunity of evaluating children who received previous therapy with drugs that can be affected by P-glycoprotein and no obvious difference in its expression was evident. Only one of the three tumour samples coming from patients who had been exposed to P-glycoprotein mediated chemotherapeutic agents (vincristine and/or etoposide) were positive in retinoblastoma cells. In addition, of the six cases in which Pglycoprotein was detected in tumour associated endothelial cells, only one of them came from a patient who had been exposed to P-glycoprotein mediated chemotherapeutic agents (etoposide). Also, one patient whose tumour and tumour associated endothelium were negative had endothelial cells in the optic nerve that were positive. The patient had not been exposed to P-glycoprotein mediated chemotherapy.

There is little information regarding its expression in normal eye blood vessels, but Pglycoprotein expression has been detected in the normal human retinal pigment epithelium and post-laminar optic nerve, ${ }^{2}$ and it is absent in the permeable microvessels of the choroid and the ciliary process. ${ }^{3}$ P-glycoprotein expression in endothelial cells of newly formed capillaries induced by tumours has also been described. ${ }^{4}$ Its prognostic relevance in retinoblastoma is unknown, and it cannot be determined from our data, since all of these eyes were already enucleated and no patient had an extraocular relapse.

The potential role of P-glycoprotein in tumour associated endothelium creating a blood-tumour barrier is uncertain. Our results suggest that if cyclosporine is indeed improving the efficacy of chemotherapy, it may be acting by improving its delivery through P-glycoprotein expressing tumour associated endothelial cells or through altering pharmacokinetics of chemotherapeutic drugs, rather than exclusively acting to reverse drug resistance at the level of the tumour cell.

Our data do not support the hypothesis that cyclosporine will be a useful adjunct to chemotherapy for the treatment of retinoblastoma via competitive inhibition of $\mathrm{P}$ glycoprotein in tumour cells.

I J Dunkel, D H Abramson, C Cordon-Cardo Memorial Sloan Kettering Cancer Center, Box 185, 1275 York Avenue New York, NY 10021, USA

G L Chantada, M T G de Davila, A C Fandino Hospital JP Garrahan, Combate de los Pozos 1881, C1245AAL, Buenos Aires, Argentina

Correspondence to: Dr Ira J Dunkel, Memorial Sloan Kettering Cancer Center, Box 185, 1275 York Avenue, New York, NY 10021, USA; dunkeli@mskcc.org
Disclosure: There is no financial interest of the authors with any of the drugs and devices mentioned. doi: $10.1136 /$ bjo.2005.068536

Accepted 8 February 2005

\section{References}

1 Krishnakumar S, Mallikarjuna K, Desai N, et al. Multidrug resistant proteins: P-glycoprotein and lung resistance protein expression in retinoblastoma. Br J Ophthalmol 2004;88:1521-6.

2 Kennedy B, Mangini N. P Glycoprotein expression in human retinal pigment epithelium. $\mathrm{Mol}$ Vis 2002;8:422-30.

3 Hofman P, Hoyng P, vander Werf F, et al. Lack of blood-brain barrier properties in microvessels of the prelaminar optic nerve head. Invest Ophthalmol Vis Sci 2001;42:895-901.

4 Camassei FD, Arancia G, Cianfriglia M, et al. Nephroblastoma: multidrug resistance $P$ glycoprotein expression in tumor cells and intratumoral capillary endothelial cells. Am J Clin Pathol 2002;117:484-90.

\section{Who is the author?}

We read with great interest Schwab's answer' to "Who is Ivan Schwab?" "We thank him for his gracious response to our original letter, ${ }^{3}$ which suggested that readers would benefit if the $B J O$ consistently published authors' qualifications. Although Schwab and the editors understood this to be a question of credentials to write about a subject, we maintain that this issue has more to do with giving the reader perspective. An excellent example of the need to understand the authors' perspective comes from Schwab's reply itself.

Schwab made the presumption that we were both ophthalmologists, which is, in fact, not correct. One of us (DP) is a medical graduate and currently a postgraduate research student. Given that the BJO does not publish authors' qualifications, it is clear how this occurred. We propose that it is common for readers to make inferences about the authors, as Schwab did. It is reasonable and logical to consider not only the information, but its source as well. When authors' qualifications are not explicitly stated, as in the $B J O$, readers can easily make erroneous presumptions about the source. Does the BJO expect its readers to make no presumptions about the backgrounds of the authors? This would seem a difficult task for the inquisitive mind. Does it matter that readers may make erroneous presumptions about the authors? We will leave this question to the reader.

It is interesting to note that, on occasion, the $B J O$ does take care to give the reader more background on an author. For example, in an article on informed consent, ${ }^{4}$ the authors are given the designations of "solicitor" and "ophthalmologist," respectively. Similarly, the author of an article on professional regulation, ${ }^{5}$ is given the designation "Chairman of Committee on Professional Performance, General Medical Council, Portex Professor of Anaesthesia, Institute of Child Health, London." This supports our view that it is important for the reader to understand the perspective of the author. Thus, the $B J O^{\prime}$ s approach does not appear consistent.

In answer to Schwab: indeed, we are "reading his essays, asking questions" and will "stay tuned." In fact, we hope that in due course, the $B J O$ will publish his series as a collection. We understand that the need to conserve space limits references. However, rather than for verification, readers who have been stimulated by his writing would benefit from more references-perhaps online-to further their understanding. For example, in August 2003, Schwab referred to Land and Nilsson's book Animal Eyes (Oxford: Oxford University Press, 2002). This is an excellent resource to improve the reader's understanding and appreciation of his essays, particularly those describing invertebrate eyes.

Finally, we note that other BJO readers have not commented on the subject of authors' qualifications. Perhaps, ironically, other readers think that they do not have the credentials to comment.

D Papalkar, I C Francis

Department of Ophthalmology, Prince of Wales Hospital, Randwick, NSW, Australia and the University of NSW, Sydney, NSW, Australia

Correspondence to: Dr lan C Francis, Suite 12, Chatswood Grove, 12-14 Malvern Avenue, Chatswood 2067, Australia; if@student.unsw.edu.au

doi: 10.1136/bjo.2005.069104

Accepted for publication 15 February 2005

\section{References}

1 Schwab IR. In answer to "Who is Ivan Schwab?" Br J Ophthalmol, 2005;89:249.

2 Hoyt CS, Dick A, Bhisitkul B. Who is Ivan Schwab? Br J Ophthalmol 2004;88:1 106.

3 Papalkar D, Francis IC. Authors' qualifications and the BJO. Br J Ophthalmol 2004;88:1227.

4 Teuten B, Taylor D. "Don't worry my good man you won't understand our medical talk": consent to treatment today. Br J Ophthalmol 2001;85:894-6

5 Hatch D. Professionally led regulation in medicine. Br J Ophthalmol 2001;85:513-15.

\section{Vitrectomy with and without scleral buckle for inferior retinal detachment}

In the article presented by Wickham and associates, ${ }^{1}$ the authors compared vitrectomy and gas for treating inferior break retinal detachments with vitrectomy, gas and scleral buckle. The study showed no significant difference in the final outcome between the two groups. While vitrectomy and gas for inferior break retinal detachments appears promising, there are several issues that we would like to raise.

Firstly, the surgery was performed by a registrar, fellow, or consultant. These surgeons may have varying degrees of experience and the inconsistency may affect the rate of successful surgical outcome. Secondly, additional tears were treated with cryotherapy or laser. As shown by Bonnet et al, ${ }^{2}$ the postoperative proliferative vitreoretinopathy (PVR) rate could be as high as $25.8 \%$ in patients treated with cryotherapy compared to $2.2 \%$ in the laser group. It is unclear what the relative distribution of patients who underwent cryotherapy in the two groups was and this may have been a confounding factor in the study. Thirdly, patients underwent an air/gas exchange with either $\mathrm{SF}_{6}$ or $\mathrm{C}_{3} \mathrm{~F}_{8}$. As $\mathrm{C}_{3} \mathrm{~F}_{8}$ had a much longer duration of tamponade than $\mathrm{SF}_{6}$, the use of one agent over another may have led to a difference in the success rate.

The study excluded patients with PVR grade C. However, for those with grade A or $\mathrm{B}$, a scleral buckle was planned before the operation. This could lead to a selection bias where potentially more difficult cases were 
scheduled into the scleral buckle group. This may be a contributing factor for a higher rate of postoperative PVR (20\%) and epiretinal membrane formation in this group, compared to a rate of $5-10 \%$ reported previously. ${ }^{34}$ The underlying vitreoretinal pathology rather than the placement of the scleral buckle may have been a major reason behind the high PVR rate noted in this group.

The authors stated that the main reasons for performing vitrectomy and gas without scleral buckle was to avoid the possible complications of scleral buckle-namely, longer operating time, ${ }^{5}$ exposure, refractive change, diplopia, and anterior segment ischaemia. ${ }^{6-10}$ Perhaps, in the interest of readers, the authors can provide us with the information if any of these complications developed during the study.

The high rate of final reattachment reported in the study is encouraging. We believe that vitrectomy and gas alone is an effective method to treat selected cases of retinal detachments with inferior retinal breaks. A controlled, randomised, prospective study, comparing the outcome in properly matched groups and with meticulous attention to surgical methods ${ }^{11}$ will help address some of the above issues and help elucidate further if the procedure without the use of scleral buckle will benefit patients with inferior break retinal detachment.

We would like to commend the authors for conducting this very nice study on an important topic that may provide a better alternative treatment. We wish that the issues that we raise will help broaden the discussion on the topic.

Y Y Y Kwong, C W Tsang, W W Lai, D S C Lam Hong Kong, People's Republic of China

Correspondence to: Professor Dennis Lam, 3/F, Hong Kong Eye Hospital, 147K Argyle Street, Kowloon, Hong Kong; dennislam_pub@cuhk.edu.hk

doi: 10.1136/bjo.2005.068528

Accepted for publication 8 February 2005

\section{References}

1 Wickham L, Connor M, Aylward GW. Vitrectomy and gas for inferior break retinal detachments: are the results comparable to vitrectomy, gas, and scleral buckle? $\mathrm{Br} J$ Ophthalmol 2004;88:1376-9.

2 Bonnet M, Guenoun S. Surgical risk factors for severe postoperative proliferative vitreoretinopathy in retinal detachment with grade B PVR. Graefes Arch Clin Exp Ophthalmol 1995;233:789-91.

3 Charteris DG, Sethi CS, Lewis GP, et al. Proliferative vitreoretinopathy. $V$ Developments in adjunctive treatment and retinal pathology. Eye 2002; 16:369-74.

4 The Retina Society Terminology Committee. The classification of retinal detachment with proliferative vitreoretinopathy. Ophthalmology 1983;90:121-5.

5 Hakin KN, Lavin MJ, Leaver PK. Primary vitrectomy for rhegmatogenous retinal detachment. Graefes Arch Clin Exp Ophthalmol 1993;231:344-6.

6 Findall RJ, Norton EW, Curtin, et al. Reduction of extrusion and infection following episcleral silicone implants and cryopexy in retinal detachment surgery. Am J Ophthalmol 1971;71:835-7.

7 Hayashi H, Hayashi K, Nakao F, et al. Corneal shape changes after scleral buckling surgery. Ophthalmology 1997;104:831-7.

8 Domniz Y, Cahana M, Avni I. Corneal surface changes after pars plana vitrectomy and scleral buckling surgery. J Cataract Refract Surg 2001;27:868-72.
9 Fison PN, Chignell AH. Diplopia after retinal detachment surgery. Br J Ophthalmol 1987;71:521-5

10 Kwartz J, Charles S, Mc Cormack P, et al. Anterior segment ischaemia following segmental scleral buckling. Br J Ophthalmol 1994;78:409-10.

11 Leaver P. Expanding the role of vitrectomy in retinal reattachment surgery. $\mathrm{Br} J$ Ophthalmol 1993;77:197.

\section{Charles Bonnet syndrome in patients with glaucoma and good acuity}

We read with interest the paper by Tan et al on Charles Bonnet syndrome (CBS) in Asian patients. Their finding of a lower CBS prevalence than European or North American surveys demands further investigation, although this may reflect the stringent criteria of hallucination complexity they used in making the diagnosis (thus excluding the commonest CBS hallucinations of coloured blobs and grid-like "tesselloptic" patterns ${ }^{2}{ }^{3}$ ) and, as pointed out in the accompanying editorial comment, the relatively low prevalence of macular disease in their cohort. However, it is not this aspect of the report we found most intriguing-it was the observation that CBS occurred with good acuity. In fact, three of the four CBS patients described had a degree of impairment which placed them at risk for CBS (best eye acuity 0.3 or worse $^{4}$ ). It is the remaining patient (patient three, a 72 year old man) who is of particular importance as his relative preservation of acuity bilaterally (20/30 RE, 20/40 LE) challenges the view that significant acuity loss is a prerequisite for "ophthalmological" visual hallucinations. This case mirrors four patients we have recently studied with CBS secondary to glaucoma and bilaterally good acuity. We describe the cases below and offer a pathophysiological mechanism for the association.

In one sense, the finding that CBS occurs with preserved acuity is hardly novel. As cited by Tan et al, ${ }^{1}$ several previous reports have found such an association. However, all is not as it seems, the term CBS being used in different ways by different authors. Some use the term to describe visual hallucinations with insight, irrespective of the presence of eye disease, age or clinical context. ${ }^{5}$ Others use the term to describe the association of visual hallucinations with age and intact cognition, without reference to eye disease or hallucination phenomenology. ${ }^{78}$ Under these definitions it is hardly surprising that a patient with "CBS" has preserved acuity, the patients in these studies having a diverse range of conditions from delirium to Parkinson's disease and beyond. In contrast, ophthalmologists and neurologists have used CBS to emphasise eye or visual pathway disease, with the phenomenology of the hallucinations and age being of secondary importance. ${ }^{910}$ Although each definition of CBS has its merits, the ophthalmological definition reminds us best of Bonnet's original description and helps characterise a distinctive subgroup of visually hallucinating patients with predicable prognosis and specific pathophysiology. ${ }^{211}$ However, even CBS as defined ophthalmologically carries with it an inherent ambiguity: is it eye disease itself or the loss of acuity that is the important factor? The consistent finding of acuity loss as a risk factor ${ }^{42}{ }^{13}$ suggests the latter, or at least that the central retina has a key role in the underlying pathophysiological mechanism.

As part of a larger study into the visual phenomenology of CBS, we have recruited four patients with advanced glaucoma (three POAG and one chronic narrow angle) but preserved visual acuity. The age range of the patients was 81-91 years, three men and one woman. Their visual acuities ranged from $6 / 6$ to $6 / 12$ monocularly with all patients having $6 / 9$ or better in their better eye. All had extensive field defects bilaterally and cup to disc ratios of 0.8 or greater in both eyes. Two patients had bilateral trabeculectomies now off treatment, one was on bimatoprost and Trusopt to both eyes and one on timolol $0.25 \%$ to both eyes. Two patients were bilaterally pseudophakic. The patient with chronic narrow angle glaucoma had previous surgical iridectomies. The duration of their hallucinations ranged from 6 months to 6 years. Three patients hallucinated in colour and one in black and white. The most common hallucination was of tessellopsia ${ }^{2}$ experienced by all the patients, with two patients seeing, in addition, formed buildings and two patients, letter-like shapes. There were also single reports of hallucinations of groups of people, animals, branching shapes $\left(\right.$ dendropsia $\left.^{2}\right)$ and one patient described visual allesthesia. ${ }^{14}$ In three of the patients the hallucinations encompassed the entire visual field, in the fourth they were restricted to the visual field defect. None had hallucinations in other sensory modalities and all had insight into the nature of the experiences. The phenomenology of the hallucinations and the relative frequency of the different hallucination categories are consistent with previous descriptions of ophthalmologically defined CBS. ${ }^{23}$ Nonophthalmological causes of visual hallucinations ${ }^{23}$ were excluded. As far as we are aware this is the largest case series of patients with visual hallucinations secondary to eye disease and bilaterally preserved visual acuity yet to be reported.

Current aetiological theories of CBS emphasise the importance of deafferentation $^{15}$ (both "physiological" through ganglion cell loss and "functional," for example, related to blindfolding or cataract), the loss of visual input resulting in a change in cortical excitability. ${ }^{2}$ Although it has been assumed that deafferentation of sufficient severity to precipitate CBS implies a consequent loss of acuity, our cases and that of Tan et $a l^{1}$ suggest otherwise. Patients with advanced glaucoma can have a significant degree of ganglion cell loss and consequent physiological deafferentation without a loss of acuity, placing them at risk for CBS. This contrasts with age related macular disease where the loss of central retinal ganglion cells leads, indirectly, to an association of CBS with acuity loss. We conclude that reduced acuity is not a necessary prerequisite for ophthalmologically defined CBS and that ophthalmologists should be aware that patients with preserved acuity but significant deafferenting ocular disease are at risk of the syndrome.

S A Madill, D H Ffytche

Eye Department, King's College Hospital, Denmark Hill, London SE5 9RS, UK

Correspondence to: Mr Stephen Madill, Eye

Department, King's College Hospital, Denmark Hill, London SE5 9RS, UK; samadill@hotmail.com

doi: 10.1136/bjo.2005.066027 
Accepted for publication 4 January 2005

\section{References}

1 Tan CSH, Lim VSY, Ho DYM, et al. Charles Bonnet syndrome in Asian patients in a tertiary ophthalmic centre. Br J Ophthalmol 2004;88: 1325-9.

2 ffytche DH, Howard RJ. The perceptual consequences of visual loss: positive pathologies of vision. Brain 1999; 122:1247-60.

3 Santhouse AM, Howard RJ, ffytche DH. Visual hallucinatory syndromes and the anatomy of the visual brain. Brain 2000;123:2055-64.

4 Teunisse RJ, Cruysberg JR, Verbeek AL, et al. The Charles Bonnet syndrome: a large prospective study in the Netherlands. Br J Psychiatry 1995; 166:254-7.

5 Damas-Mora J, Skelton-Robinson M, Jenner FA. The Charles Bonnet syndrome in perspective. Psychol Med 1982;12:251-61.

6 Gold K, Rabins PV. Isolated visual hallucinations and the Charles Bonnet syndrome: a review of the literature and presentation of six cases. Compr Psychiatry 1989;30:90-8.

7 Podoll K, Osterheider M, Noth J. Das Charles Bonnet-Syndrom. Fortschr. Neurol Psychiat 1989;57:43-60.

8 De Morsier G. Le syndrome de Charles Bonnet: hallucinations visuelles des vieillards sans deficience mentale. Annales MedicoPsychologiques 1967;125:677-702.

9 Manford M, Andermann F. Complex visual hallucinations. Clinical and neurobiological insights. Brain 1998;121:1819-40.

10 Menon GJ, Rahman I, Menon SJ, et al. Complex visual hallucinations in the visually impaired: the Charles Bonnet syndrome. Surv Ophthalmol 2003;48:58-72.

11 ffytche DH. Visual hallucination and illusion disorders: a clinical guide. Adv Clin Neurosci Rehab 2004;4:16-18.

12 Holroyd S, Rabins PV, Finkelstein D, et al. Visual hallucinations in patients with macular degeneration. Am J Psychiatry 1992;149:1701-6.

13 Scott IU, Schein OD, Feuer WJ, et al. Visual hallucinations in patients with retinal disease. Am J Ophthalmol 2001;131:590-8.

14 Girkin CA, Miller NR. Central disorders of vision in humans. Surv Ophthalmol 2001;45:379-405.

15 Burke W. The neural basis of Charles Bonnet hallucinations: a hypothesis. J Neurol Neurosurg Psychiatry 2002;73:535-41.

\section{Author's reply}

I thank Madill and Ffytche for their interesting comments on our paper ${ }^{1}$ and am grateful for the opportunity to respond to some of the issues raised. The first relates to possible variations in the prevalence rate of Charles Bonnet syndrome (CBS) in different populations, on which there is currently very little substantive data. We agree that additional population based studies are necessary to determine whether the differences found between ethnic groups are consistent. We wish to highlight that our findings ${ }^{1}$ are supported by another recently published study of 1000 Japanese patients by Shiraishi et $\mathrm{al}^{2}$ who reported a prevalence rate of $0.5 \%$ compared to $0.4 \%$ in our study population. Since the methodology and diagnostic criteria used in that study are very similar to ours, the comparable prevalence rates reported in both studies may be a true reflection of a lower prevalence of CBS in Asians compared to Western populations.

We would like to point out that the large variation in prevalence rates of CBS in the various cross sectional studies may be explained by the characteristics of the different populations being screened, especially the degree of visual impairment. When patients were drawn from either a general ophthalmology $^{1-3}$ or general medical ${ }^{4}$ clinic, presumably comprising patients with relatively good visual acuity, the prevalence rates ranged from $0.4-2 \%$. In patients with poorer visual acuity ${ }^{35-8}$ the prevalence rates varied from $11-12.3 \%$. This observation suggests that poor visual acuity may be a factor that increases the risk of developing CBS. Indeed, Teunisse et al showed that the risk of CBS was higher in patients with a logMAR visual acuity of worse than $0.3^{3}$ and Holroyd et al also found an association with bilateral visual acuity of 20/60 or less. ${ }^{9}$

Nevertheless, even if reduced visual acuity is indeed a risk factor for the development of CBS, we agree that a reduction in visual acuity is not essential and that CBS can occur in patients with relatively good visual acuity. Gold et al thought that reduced visual acuity is not a prerequisite for the diagnosis of $\mathrm{CBS}^{10}$ and Holroyd et al suggested that it may be the presence of visual impairment and not the severity of visual loss that predisposes to CBS. ${ }^{4}$ Schultz and Melzack, in an excellent review of the cases reported in the literature, found that the visual acuities of patients diagnosed with CBS ranged from normal to no light perception. ${ }^{11}$ It is becoming apparent that CBS can develop in patients with visual field defects but otherwise good central acuity. The patients described by Madill and Ffytche and our own patient (patient 3) had glaucomatous visual field defects. A recent paper by Freiman et $a l^{12}$ documented the presence of hallucinations manifesting within visual field defects that occurred following neurosurgery. Two interesting points can be noted from Freiman et al's series: firstly, the hallucinations were confined to the area of visual loss; secondly, they began soon after the surgery and resolved within days to 6 months after their onset. ${ }^{4}$ In contrast, in three of four patients reported by Madill and Ffytche as well as our own patient, the hallucinations were not restricted to the visual field defect. The relation of the onset of CBS symptoms to the development of visual field defects as well as the localisation of hallucinations within visual fields are issues that warrant further study as they may provide additional insight into the pathophysiology of CBS.

Correspondence to: Colin S H Tan, The Eye Institute, National Healthcare Group, Singapore; colintan_eye@yahoo.com.sg

doi: $10.1136 /$ bjo.2005.068510

Accepted for publication 8 February 2005

\section{References}

1 Tan CSH, Lim VSY, Ho DYM, et al. Charles Bonnet syndrome in Asian patients in a tertiary ophthalmic centre. Br J Ophthalmol 2004:88:1325-9.

2 Shiraishi Y, Terao T, lbi K, et al. The rarity of Charles Bonnet syndrome. J Psychiatr Res 2004;38:207-13.

3 Teunisse RJ, Cruysberg JR, Verbeek AL, et al. The Charles Bonnet syndrome: a large prospective study in the Netherlands. Br J Psychiatry 1995; 166:254-7.

4 Holroyd S, Rabins PV, Finkelstein D, et al. Visual hallucinations in patients from an ophthalmology clinic and medical clinic population. J Nerv Ment Dis 1994; 182:273-6.

5 Brown GC, Murphy RP. Visual symptoms associated with choroidal neovascularization. Photopsias and the Charles Bonnet syndrome. Arch Ophthalmol 1992;110:1251-6.
6 Olbrich HM, Engelmeier MP, Pauleikhoff D, et al. Visual hallucinations in ophthalmology. Graefes Arch Clin Exp Ophthalmol 1987;225:217-20.

7 Teunisse RJ, Cruysberg JR, Hoefnagels WH, et al. Visual hallucinations in psychologically normal people: Charles Bonnet's syndrome. Lancet 1996;347:794-7.

8 Nesher R, Nesher G, Epstein E, et al. Charles Bonnet syndrome in glaucoma patients with low vision. J Glaucoma 2001;10:396-400.

9 Holroyd S, Rabins PV, Finkelstein D, et al. Visual hallucinations in patients with macular degeneration. Am J Psychiatry 1992;149:1701-6.

10 Gold K, Rabins PV. Isolated visual hallucinations and the Charles Bonnet syndrome: a review of the literature and presentation of six cases. Compr Psychiatry 1989;30:90-8.

11 Schultz G, Melzack R. The Charles Bonnet syndrome: 'phantom visual images'. Perception 1991;20:809-25.

12 Freiman TM, Surges R, Vougioukas VI, et al. Complex visual hallucinations (Charles Bonnet syndrome) in visual field defects following cerebral surgery. Report of four cases. J Neurosurg 2004;101:846-53.

\section{Tight necktie, intraocular pressure, and intracranial pressure}

I would like to congratulate Theelen et al for their recent article on impact factors on intraocular pressure measurements in healthy subjects, ${ }^{1}$ and I would like to add a thought. As Theelen and colleagues point out with reference to the literature, ${ }^{23}$ increased pressure in the jugular vein leads to increased brain pressure, and by an increase in the episcleral venous pressure, to an elevation of intraocular pressure. Correspondingly, in a previous study by Teng and associates, it was discussed that a tight necktie may increase intraocular pressure by an increased jugular vein pressure and could affect the diagnosis and management of glaucoma. ${ }^{4}$ It may be taken into account, however, that the brain pressure and pressure in the cerebrospinal fluid space surrounding the retrobulbar part of the optic nerve are the counter-pressure against the intraocular pressure across the lamina cribrosa. ${ }^{5}$ If the cerebrospinal fluid space pressure is elevated (as a result of increased jugular vein pressure), the intraocular pressure may also be allowed to be elevated so that the trans-lamina cribrosa pressure difference may remain constant. Independently of the question of whether a tight necktie may or may not increase intraocular pressure, one may assume that if the intraocular pressure gets higher because of an increased jugular vein pressure, it may, at least partially, be balanced by an increase in brain pressure, without increasing the risk for glaucoma.

Correspondence to: J B Jonas, Department of Ophthalmology, Theodor-Kutzer-Ufer 1-3 Mannheim, 68167, Germany; jost.jonas@augen.ma.uni-heidelberg.de doi: $10.1136 /$ bjo.2005.068544 Accepted for publication 8 February 2005

\section{References}

1 Theelen T, Meulendijks CFM, Geurts DEM, et al Impact factors on intraocular pressure measurements in healthy subjects. Br J Ophthalmol 2004;88:1510-1.

2 Mavrocordatos P, Bissonnette B, Ravussin P. Effects of neck position and head elevation on intracranial pressure in anaesthetized 
neurosurgical patients - preliminary results. J Neurosurg Anesthesiol 2000;12:10-4.

3 Toole JF. Effects of change of head limb and body position on cephalic circulation. N Engl J Med 1968:279.307-11.

4 Teng C, Gurses-Ozden R, Liebmann JM, et al. Effect of a tight necktie on intraocular pressure. Br J Ophthalmology 2003:87:946-94.

5 Jonas JB, Berenshtein E, Holbach L. Lamina cribrosa thickness and spatial relationships between intraocular space and cerebrospinal fluid space in highly myopic eyes. Invest Ophthalmol Vis Sci 2004:45:2660-5.

\section{Authors' reply}

We thank Dr Jonas for his interest in our work and his important supplements about pathophysiological aspects regarding neck circulation and intraocular pressure. As the optic nerve head is in close contact with both intraocular and subarachnoidal space, one may imagine a disc protecting balance in chronically elevated jugular pressure. ${ }^{1}$ Short term fluctuations of cervical circulation, as present in our recent study, may not facilitate this guarding benefit. ${ }^{2}$ Thus, repeated huge variations of jugular pressure can presumably be a risk factor for glaucomatous optic nerve head damage. Obviously, this is not the case in slit lamp adapted measurement of intraocular pressure. Nevertheless, the patient's neck position during tonometry should be taken into consideration to improve the interpretation of intraocular pressure measurements.

T Theelen, C F M Meulendijks Radboud University Nijmegen Medical Centre, Philips van Leijdenlaan 15 Nijmegen, 6525 EX, Netherlands

Correspondence to: T Theelen, Radboud University Niimegen Medical Centre, Philips van Leijdenlaan 15 Nijmegen, 6525 EX, Netherlands t.theelen@ohk.umcn.nl doi: 10.1136/bjo.2005.069757

Accepted for publication 23 February 2005

\section{References}

1 Jonas JB, Berenshtein E, Holbach L. Lamina cribrosa thickness and spatial relationships between intraocular space and cerebrospinal fluid space in highly myopic eyes. Invest Ophthalmol Vis Sci 2004;45:2660-5.

2 Theelen T, Meulendijks CFM, Geurts DEM, et al. Impact factors on intraocular pressure measurements in healthy subjects. $\mathrm{Br} J$ Ophthalmol 2004;88:1510-11

\section{NOTICES}

Worldwide clinical trials for new technique for early detection of eye disease

A unique new non-invasive technique for high resolution optical imaging of the eye is receiving global acclaim. By combining two high-resolution imaging technologies, the new technique provides doctors with 3-D images of the retina, macula and the optic nerve.

For more information, contact the Media Office on $01227823581 / 823100$ or email MediaOffice@kent.ac.uk News releases can also be found at: http://www.kent.ac.uk/news

\section{Trachoma control}

The latest issue of Community Eye Health (No 52) discusses new developments in the control of trachoma. For further information please contact: Journal of Community Eye Health, International Resource Centre, International Centre for Eye Health, Department of Infectious and Tropical Diseases, London School of Hygiene and Tropical Medicine,
Keppel Street, London WCIE 7HT, UK (tel: +44 (0)207612 7964; email: Anita.Shah@ Ishtm.ac.uk; online edition: www.jceh.co. uk). Annual subscription (4 issues) UK $£ 28$ / US\$45. Free to developing country applicants.

\section{EVER 2005 meeting}

This will take place on 5-8 October 2005 in Vilamoura, Portugal. For further details please contact: Christy Lacroix, EVER Secretary, Kapucijnenover 33, B-3000 Leuven, Belgium (tel: +32 (0) 16233 849; fax +32 (0)16 234 097; email:ever@ skynet.be).

\section{World Ophthalmology Congress 2006 - Brazil}

The World Ophthalmology Congress (which is replacing the International Congress of Ophthalmology) is meeting in February 2006 in San Paulo, Brazil.

For further information on the congress and committees, scientific program and coordinators of different areas are available at the congress website www.ophthalmology2006. com.br

\section{CORRECTION}

doi: 10.1136/bjo.2005.48777corr l

In the paper titled Prevalence and causes of blindness and low vision in leprosy villages of north eastern Nigeria ( $\mathrm{Br} J$ Ophthalmol 2005;89:417-9) the author has notified us of an error. The second-last sentence in the second column on page 417 should read, 'Definitions of blindness and visual impairment were: VA $\geqslant 6 / 18$, normal; $6 / 60 \leqslant \mathrm{VA}<$ $6 / 18$, visual impairment; $3 / 60 \leqslant \mathrm{VA}<6 / 60$, severe visual impairment...'. 